\title{
THE FREE GROTHENDIECK THEOREM
}

\author{
MERIC L. AUGAT
}

\begin{abstract}
The main result of this article establishes the free analog of Grothendieck's Theorem on bijective polynomial mappings of $\mathbb{C}^{g}$. Namely, we show if $p$ is a polynomial mapping in $g$ freely noncommuting variables sending $g$-tuples of matrices (of the same size) to $g$-tuples of matrices (of the same size) that is injective, then it has a free polynomial inverse.

Other results include an algorithm that tests if a free polynomial mapping $p$ has a polynomial inverse (equivalently is injective; equivalently is bijective). Further, a class of free algebraic functions, called hyporational, lying strictly between the free rational functions and the free algebraic functions are identified. They play a significant role in the proof of the main result.
\end{abstract}

\section{INTRODUCTION}

A remarkable pair of theorems of Grothendieck [Gro66, Gro67] say if $p: \mathbb{C}^{g} \rightarrow \mathbb{C}^{g}$ is an injective polynomial, then $p$ is bijective and its inverse is a polynomial. Later degree bounds on the inverse were discovered. These results are of course intimately connected with the fascinating Jacobian conjecture (see for instance [BCW82, vdE00]) and the question of tame versus wild automorphisms of the polynomial ring (see for instance [SU03, SU04, UY04, Umi06]).

In this article we prove the free Grothendieck theorem. Our approach involves careful analysis of the noncommutative Jacobian matrix as found in [Reu92], the theories of free rational series [BR11] and their realizations [KV17, Vol15], formal power series in noncommuting variables [Sta11], free analysis [HM04, HKM11], proper algebraic systems [SS78, PS09], free derivatives[Pas14] and skew fields [Coh95]. We also make use of some new machinery including the hyporational functions and the hypo-Jacobian matrix defined later in this paper.

To state the result, fix a positive integer $g$ and let $\boldsymbol{x}=\left(x_{1}, \ldots, x_{g}\right)$ denote a tuple of freely noncommuting indeterminants. A free polynomial (in $g$-variables) is a finite $\mathbb{C}$ linear combination of words in $x$. For positive integers $n$, let $M_{n}(\mathbb{C})^{g}$ denote the $g$-tuples of $n \times n$ matrices over $\mathbb{C}$ and let $M(\mathbb{C})^{g}$ denote the sequence $\left(M_{n}(\mathbb{C})^{g}\right)_{n}$. A free polynomial $f$ induces a sequence of maps $f[n]: M_{n}(\mathbb{C})^{g} \rightarrow M_{n}(\mathbb{C})$ by evaluation, $X \mapsto f(X)$. We let $f: M(\mathbb{C})^{g} \rightarrow M(\mathbb{C})$ denote this sequence. A free polynomial mapping $p: M(\mathbb{C})^{g} \rightarrow M(\mathbb{C})^{g}$ is thus a $g$-tuple of sequences, $p=\left(p^{1}, \ldots, p^{g}\right)$, that is, each $p^{i}$ is a free polynomial. The polynomial mapping $p$ is injective (resp. surjective, bijective) if each $p[n]$ is injective (resp. surjective, bijective). Of course if $p[n]$ is injective, then considered as a polynomial in $g n^{2}$ commuting variables, it is bijective and has a polynomial inverse. The following free polynomial analog of Grothendieck's Theorem was implicitly conjectured in [Pas14].

Theorem 6.22 ((Free Grothendieck Theorem)). If $p: M(\mathbb{C})^{g} \rightarrow M(\mathbb{C})^{g}$ is an injective free polynomial mapping, then there is a free polynomial mapping $q$ such that $p \circ q(x)=x=q \circ p(x)$; that is, $p$ has a free polynomial inverse. 
Before describing our methods in further detail, we pause to note that Theorem 6.22 is of course related to the study of automorphisms, and the question of tame versus wild automorphisms of the free algebra (see for instance [Dic82, DY07, DY06, ML70, Umi07]). Pascoe [Pas14] proves a free (freely noncommutative) inverse function theorem and uses this theorem to establish a free analog of the Jacobian conjecture, stated below.

Theorem A (Free Jacobian Conjecture). Suppose $p: M(\mathbb{C})^{g} \rightarrow M(\mathbb{C})^{g}$ is a free polynomial mapping. The following are equivalent:

(i) $D p(X)[H]$ is nonsingular for each $X \in M(\mathbb{C})^{g}$; that is, for each positive integer $n$ and each tuple $X \in M_{n}(\mathbb{C})^{g}$, the linear mapping $M_{n}(\mathbb{C})^{g} \ni H \mapsto$ $D p(X)[H]$ is non-singular;

(ii) $p$ is injective;

(iii) $p$ is bijective;

(iv) $p^{-1}$ exists as a free function, and for each $n,\left.p^{-1}\right|_{M_{n}(\mathbb{C})^{g}}$ agrees with a free polynomial mapping; namely, there exists a free mapping $g: M(\mathbb{C})^{g} \rightarrow$ $M(\mathbb{C})^{g}$ and free polynomial mappings $q_{n}$ such that $p(g(X))=X=g(p(X))$ for all $X \in M(\mathbb{C})^{g}$ and $g(X)=q_{n}(X)$ for each $n$ and $X \in M_{n}(\mathbb{C})^{g}$.

The notion of a free function is defined in Subsection 2.1 and the free derivative $D p$ is defined in Subsection 4.2. We will often use the equivalence of items (ii) and (iii).

Three results in this article require little or no additional overhead to state. Assuming $p$ is injective, Theorem 7.5 produces bounds for the degree of its inverse $q$. As a concrete example, $\operatorname{deg}(q) \leq\left(3^{g} \prod_{i=1}^{g} i^{3}\right)(\operatorname{deg}(p)-1)+1$. Using the degree bound, Corollary 7.7 describes an algorithm that takes as input a free polynomial $p$ and, after a number of iterations depending only on the number of variables $g$ and the degree of $p$, either produces a polynomial $q$ - the inverse of $p$ - or $p$ is not injective and has no polynomial inverse.

The derivative $D p(\boldsymbol{x})[\boldsymbol{h}]$ is a $g$-tuple of polynomials in the $2 g$ freely noncommuting variables $(\boldsymbol{h}, \boldsymbol{x})=\left(h_{1}, \ldots, h_{g}, x_{1}, \ldots, x_{g}\right)$ defined as the free analog of the directional derivative in the obvious way. The result of Pascoe mentioned above $-p$ is bijective if and only if $\boldsymbol{h} \mapsto D p(\boldsymbol{x})[\boldsymbol{h}]$ is pointwise non-singular - is strengthened by the following result.

Corollary 6.14. A free polynomial $p: M(\mathbb{C})^{g} \rightarrow M(\mathbb{C})^{g}$ is bijective if and only if $(\boldsymbol{h}, \boldsymbol{x}) \mapsto(D p(\boldsymbol{x})[\boldsymbol{h}], \boldsymbol{x})$ has a polynomial inverse.

We use the free derivative to state and prove Theorem 4.8, the implicit function theorem for nc formal power series. It is mostly a consequence of Lemma 3.9. We refer to [AM16] for an in depth analysis of the implicit function theorem for several topologies on $M(\mathbb{C})^{g}$.

Theorem 4.8 ((Implicit function theorem)). Suppose $f(\boldsymbol{x}, \boldsymbol{z}) \in\left(\mathbb{C}\langle\langle\mathbb{x} \cup \mathbb{Z}\rangle)^{h}\right.$. If $f(0,0)=0$ and $\partial f / \partial \boldsymbol{z}(0,0) \in M_{h}(\mathbb{C})$ is invertible, then there exists a unique $\mathfrak{g} \in(\mathbb{C}\langle\langle\mathbb{x}\rangle\rangle)^{h}$ such that $\mathfrak{g}(0)=0$ and $f(\boldsymbol{x}, \mathfrak{g}(\boldsymbol{x}))=0$.

1.1. The Jacobian, free algebraic functions and proper algebraic systems. The left Jacobian matrix [Reu92] of a free polynomial mapping $p: M(\mathbb{C})^{g} \rightarrow$ $M(\mathbb{C})^{g}$ with no constant term is the unique $g \times g$ matrix $J_{p}$ with free polynomial entries such that

$$
p(\boldsymbol{x})=\left(\begin{array}{lll}
p^{1}(\boldsymbol{x}) & \cdots & p^{g}(\boldsymbol{x})
\end{array}\right)=\boldsymbol{x} J_{p}(\boldsymbol{x})=\left(\begin{array}{lll}
\boldsymbol{x}_{1} & \cdots & \boldsymbol{x}_{g}
\end{array}\right) J_{p}(\boldsymbol{x}) .
$$


In particular,

$$
p^{j}(\boldsymbol{x})=\sum_{s=1}^{g} \boldsymbol{x}_{s}\left(J_{p}\right)_{s, j}(\boldsymbol{x}) .
$$

The definition of the Jacobian matrix $J_{p}$ extends naturally to the case where each $p^{j}$ is a free formal power series with no constant term. In this case $J_{p}$ is a $g \times g$ matrix with free formal power series entries. It has a multiplicative inverse if there is a $g \times g$ matrix $\mathcal{J}_{p}$ of free formal power series such that $J_{p}(\boldsymbol{x}) \mathcal{J}_{p}(\boldsymbol{x})=I_{g}$ and $\mathcal{J}_{p}(\boldsymbol{x}) J_{p}(\boldsymbol{x})=I_{g}$. In this case $\mathcal{J}_{p}$ is unique and denoted $J_{p}^{-1}$. The following proposition combines Corollary 3.5 and Lemma 3.9.

Proposition 3.12. Suppose $p$ is a free formal power series mapping without constant term. There is a free formal power series mapping $q$ (without constant term) such that $p$ and $q$ are compositional inverses if and only if $J_{p}$ has a (free formal power series) multiplicative inverse. In this case, $J_{p}(q(\boldsymbol{x}))=J_{q}^{-1}(\boldsymbol{x})$. Moreover, $q$ is the unique solution of

$$
q(\boldsymbol{x})=\boldsymbol{x} J_{p}^{-1}(q(\boldsymbol{x})) .
$$

In the case that $J_{p}^{-1}$ is a polynomial, equation (3.6) implies $q$, the inverse of $p$, is algebraic. To state the result more precisely requires a definition. Suppose $\boldsymbol{z}=\left(z_{1}, \ldots, z_{h}\right)$ is an additional tuple of freely noncommuting variables and $\alpha(\boldsymbol{x})[\boldsymbol{z}]=\left(\begin{array}{lll}\alpha^{1} & \ldots & \alpha^{h}\end{array}\right)$ is a polynomial mapping. We say $\alpha$ is a proper algebraic polynomial mapping if $\alpha$ has no constant terms and each monomial appearing in $\alpha$ with degree in $z$ of at least one, has total degree of at least two. A tuple of free formal power series without constant term, $\beta(\boldsymbol{x})=\left(\begin{array}{lll}\beta^{1} & \ldots & \beta^{h}\end{array}\right)$, is a solution to the proper algebraic polynomial $\alpha$ if

$$
\alpha(\boldsymbol{x})[\beta(\boldsymbol{x})]=\beta(\boldsymbol{x}) .
$$

We say each $\beta^{i}$ is a component of the solution. By [Sta11, Theorem 6.6.3], every proper algebraic polynomial mapping has a unique solution. A formal power series $\gamma(x)$ is algebraic if $\gamma-c$ is a component of the solution to some proper algebraic polynomial mapping.

If both $p$ and $p^{-1}$ are a polynomial mappings, then the chain rule implies $J_{p}^{-1}$ is a polynomial matrix (Remark 3.16 and [Reu92, Corollary 1.4]). Thus the following theorem follows immediately from Theorem 6.22. We give an independent proof and the result itself is a key ingredient in the proof of Theorem 6.22.

Theorem 3.17. If $p$ is a bijective polynomial mapping, then $J_{p}^{-1}$ is a polynomial (matrix).

Example 3.19 concisely points out the limitations of the Jacobian matrix; it does not detect the non-injectivity of a polynomial.

1.2. Hyporational functions. If $p$ is a bijective free polynomial, then necessarily its inverse $q$ is an algebraic mapping. If in addition, $q$ is rational, then [KV17, Theorem 4.2] implies $q$ is a polynomial. In Section 6 we identify, in terms of proper algebraic polynomial mappings, free rational functions amongst free algebraic mappings and extend, in Theorem 6.13 below, [KV17, Theorem 4.2] to a larger class of free algebraic functions.

In fact, a formal power series is rational if and only if it is a component of the solution of some proper algebraic polynomial $\alpha$ of the form,

$$
\alpha(\boldsymbol{x})[\boldsymbol{z}]=\mathfrak{a}(\boldsymbol{x})+\boldsymbol{z} \mathscr{A}(\boldsymbol{x}),
$$


where $\mathfrak{a}$ is a polynomial mapping and $\mathscr{A}$ is a polynomial matrix. On the other hand, example 6.4 shows that the solution to a proper algebraic polynomial mapping having degree one in $z$ is not necessarily rational.

A formal power series $a(\boldsymbol{x})$ with constant term $a_{\mathbb{1}}$ is a hyporational series if $a-a_{\mathbb{1}}$ is a component of the solution to a proper algebraic polynomial mapping $\alpha(\boldsymbol{x})[\boldsymbol{z}]$ of degree one in $z$. Every rational series is a hyporational series and Example 6.4 shows this inclusion is proper. Hence hyporational functions lie properly between free rational functions and free algebraic functions. The following result shows that hyporationals enjoy some of the same regularity properties as rationals.

Theorem 6.13. Suppose $a$ is hyporational. If $\operatorname{dom}_{n}(a)=M_{n}(\mathbb{C})^{g}$ for all $n$, then $a$ is a free polynomial.

In Section 6 we introduce the hypo-Jacobian matrix $J_{p}^{\text {hyp }}$ of a free polynomial mapping $p$. It is a $g \times g$ matrix whose entries are bipartite polynomials; that is polynomials in the two $g$-tuples of freely noncommuting variables $\boldsymbol{x}$ and $\boldsymbol{y}$, but where $x_{j} y_{k}=y_{k} x_{j}$ for all $1 \leq j, k \leq g$. See Lemma 6.15 and Definition 6.16. Theorem 6.18 shows that the hypo-Jacobian matrix of a free polynomial mapping is simply a matrix form of the free derivative; the hypo-Jacobian's invertibility as a matrix encodes the invertibility of free polynomials. Indeed, we obtain the following improvement of Theorem 3.17.

Theorem 6.18. The free polynomial mapping $p$ is injective if and only if $J_{p}^{\text {hyp }}$ has a multiplicative inverse whose entries are bipartite polynomials.

In other words, a free polynomial mapping $p$ is injective if and only if its hypoJacobian matrix is invertible (as a bipartite polynomial matrix).

The notion of the hypo-Jacobian matrix arises in the study of endomorphisms of the free associative algebra $\mathbb{C}\langle\mathbb{x}\rangle$. In fact, any such endomorphism has a Jacobian matrix (see [DL82] and [Sch85]) that exactly corresponds with our notion of the hypo-Jacobian matrix.

1.3. Reader's guide. Section 2 introduces definitions and notation from formal power series and free analysis that are repeatedly used throughout the paper.

The Jacobian matrix of a formal power series is defined in Section 3 and it serves as one of the central objects of study. Invertibility of the Jacobian matrix is necessary and sufficient for a formal power series mapping to have a compositional inverse (Proposition 3.12). In subsection 3.2 we borrow ideas from enumerative combinatorics - namely the construction of an algebraic formal power series by iterating the composition of a set of polynomials - and exploit the chain rule for the Jacobian matrix to iteratively construct these compositional inverses. Subsection 3.3 extends results about Jacobian matrices to free analytic functions. These results are then combined with a noncommutative Nullstellensatz - due to man [HM04] to prove the key intermediate result Theorem 3.17: if a free polynomial is injective, then its Jacobian matrix has a polynomial matrix inverse.

Subsection 4.1 explores conditions that guarantee a free polynomial has a free polynomial inverse. While in subsection 4.2 we recall the free derivative as defined in [Pas14] and investigate its properties. For a fixed free polynomial $p$, we define the function $F:(\boldsymbol{x}, \boldsymbol{y}) \mapsto(D p(\boldsymbol{y})[\boldsymbol{x}], \boldsymbol{y})$ and observe that Pascoe's solution [Pas14] to the free Jacobian conjecture can be interpreted as saying, $p$ is bijective if and only if $F$ is bijective. Setting $G$ equal to the (free) inverse of $F$, Lemma 4.11 shows 
that there is a $\boldsymbol{z}$-affine linear mapping $\mathbb{G}$ such that the first $g$ entries of $G$ are the solution to $\mathbb{G}$. Understanding the inverse function $G$ is what motivates Section 6 .

Section 5 seemingly departs from the previous discussion and establishes facts about noncommutative rational functions and rational degree maps needed in the following section. The main result of this section is Proposition 5.2. It shows that evaluating a nc rational function $r$ on matrices produces a matrix whose entries behave much like the abelianization of $r$.

Section 6 introduces the hyporationals, a generalization of rational formal power series. We proceed to show that the free derivative of an injective polynomial has a hyporational inverse. If $s$ is a hyporational series that is not rational, then we cannot apply results from realization theory. However, $s[n]=\left.s\right|_{M_{n}(\mathbb{C})^{g}}$ is a commutative rational function for each $n$, hinting that it may be possible to extend regularity results from nc rational functions to hyporational functions. Proposition 6.8 does so by constructing the hypomatrix representation of hyporational function; a matrix over $\mathbb{C}\langle\mathbb{x}\rangle \otimes \mathbb{C}\langle\mathbb{y}\rangle$, the algebra of bipartite polynomials, that imitates the realization theory of nc rational functions. The algebra $\mathbb{C}\langle\mathbb{x}\rangle \otimes \mathbb{C}\langle y\rangle$ is contained in a skew field $\mathbb{C} \nless \mathbb{x} \leftrightarrow \mathbb{y} \ngtr$, and the hypomatrix representation is invertible as a matrix over $\mathbb{C} \nless \mathbb{X} \leftrightarrow \mathbb{y} \ngtr$. Thus, we may use the results of Section 5 to analyze hyporational functions.

By applying Proposition 5.2 we prove Theorem 6.13: a hyporational function with no domain exceptions is in fact a polynomial, a result established in [KV17] and [KPV17] for free rational functions. A straightforward consequence is Corollary 6.14. It strengthens Pascoe's resolution of the Free Jacobian Conjecture by asserting: a free polynomial $p$ is injective if and only if $(\boldsymbol{h}, \boldsymbol{x}) \mapsto(D p(\boldsymbol{x})[\boldsymbol{h}], \boldsymbol{x})$ has a polynomial inverse. This corollary is both an ingredient in, and immediate consequence of, Theorem 6.22 assuming bijectivity of $F(\boldsymbol{x}, \boldsymbol{y})=(D p(\boldsymbol{y})[\boldsymbol{x}], \boldsymbol{y})$.

In subsection 6.3 we introduce $J_{p}^{\text {hyp }}$, the hypo-Jacobian matrix of the free polynomial $p$. Using Corollary 6.14 we prove Theorem 6.18: a free polynomial is injective if and only if its hypo-Jacobian matrix is invertible as a matrix of bipartite polynomials. Connecting Theorem 6.18 to results in [DL82] and [Sch85] proves the Free Grothendieck Theorem, Theorem 6.22.

Lastly, in Section 7 we discuss computational aspects of computing the free inverse $q$ of a given free polynomial $p$. If $q$ is a free polynomial then Theorem 7.5 provides an upper bound for the degree of $q$ depending only on the number of variables and the degree of $p$, leading to an algorithmic test for whether $p$ has a polynomial inverse, Lemma 7.6.

Acknowledgements. The author would like to thank his advisor Scott McCullough for reviewing the manuscript and for the many valuable discussions along the way. The author would also like to thank Igor Klep, Jurij Volčič, James Pascoe and Špela Špenko for many helpful conversations and for pointing out the existence of several algebraic results.

\section{Preliminaries}

Let $\mathcal{A}$ be any $\mathbb{C}$-algebra. We denote the $n \times n$ matrix algebra with entries in $\mathcal{A}$ by $M_{n}(\mathcal{A})$. Let $\mathbb{x}=\left\{x_{1}, \ldots, x_{g}\right\}$ be a set of noncommuting indeterminates. The set of finite sequences of elements of $\mathbb{x}$ is denoted by $\langle\mathbb{x}\rangle$. The empty sequence is the identity element of $\langle\mathbb{x}\rangle$ and is denoted by $\mathbb{1}$. 
An element of $\mathbb{x}$ is called a letter, an element of $\langle\boldsymbol{x}\rangle$ is called a word and the length of a word $w=x_{i_{1}} \ldots x_{i_{m}}$ is $m$, denoted by $|w|$. We denote the algebra of free formal power series with coefficients in $\mathcal{A}$ by $\mathcal{A}\langle\langle\mathbb{x}\rangle\rangle$ and if $f \in \mathcal{A}\langle\langle\mathbb{x}\rangle\rangle$ then

$$
f=\sum_{w \in\langle x\rangle} c_{w} w
$$

where each $c_{w} \in \mathcal{A}$.

We say $p \in \mathcal{A}\langle\langle\mathbb{x}\rangle\rangle$ is a polynomial if all but a finite number of the coefficients of $p$ are zero. The set of all polynomials, denoted $\mathcal{A}\langle\mathbf{x}\rangle$, is the familiar free algebra on $g$ noncommuting indeterminates. It is a subalgebra of $\mathcal{A}\langle\langle\mathbb{x}\rangle$. We denote the formal power series with no constant term by $\mathcal{A}\langle\langle\mathbf{x}\rangle\rangle_{+}$and the formal polynomials with no constant term by $\mathcal{A}\langle\mathbb{x}\rangle_{+}$. by

Suppose $\alpha=\sum_{w} a_{w} w$ and $\beta=\sum_{w} b_{w} w$. Define $\omega: \mathcal{A}\langle\langle\mathbb{x}\rangle\rangle \times \mathcal{A}\langle\langle\mathbb{x}\rangle \rightarrow \mathbb{N} \cup\{\infty\}$

$$
\omega(\alpha, \beta)=\inf \left\{n \in \mathbb{N}: \exists w \in\langle\mathbb{x}\rangle,|w|=n \text { and } a_{w} \neq b_{w}\right\} .
$$

The function $d: \mathcal{A}\langle\langle\mathbf{x}\rangle\rangle \times \mathcal{A}\langle\langle\mathbb{x}\rangle\rangle \rightarrow \mathbb{R}$ given by $d(\alpha, \beta)=2^{-\omega(\alpha, \beta)}$ is a metric on $\mathcal{A}\langle\langle\mathbb{x}\rangle\rangle$. Furthermore, $\mathcal{A}\langle\langle\mathbb{x}\rangle\rangle$ is complete and $\mathcal{A}\langle\mathbf{x}\rangle$ is dense in $\mathcal{A}\langle\langle\mathbb{x}\rangle\rangle$. The metric topology above is equivalent to the $(\mathbb{x})$-adic topology.

Formal power series may be generalized further to free products of unital $\mathbb{C}$ algebras. An easy example of such a power series is a polynomial $p \in \mathbb{C}\langle\mathbb{X} \cup \mathbb{Z}\rangle$, which can instead be taken as a polynomial in the free product of $\mathbb{C}\langle\mathbb{x}\rangle$ with $\mathbb{C}\langle\mathbb{Z}\rangle$. The free product of $\mathbb{C}\langle\mathbb{x}\rangle$ and $\mathbb{C}\langle\mathbb{Z}\rangle$ is the set of all words $\alpha^{1} \beta_{1} \ldots \alpha^{k} \beta_{k}$, where $\alpha^{i} \in\langle\mathbb{x}\rangle$, $\beta_{i} \in\langle\mathbb{Z}\rangle$ are nonempty words. A much more detailed exposition can be found in [Vol15].

Definition 2.1. If $S \in \mathbb{C}\langle\langle\mathbb{x}\rangle\rangle$ and $S$ has a nonzero constant term $\rho$, then $S^{-1}$ the multiplicative inverse of $S$, exists and is given by

$$
S^{-1}=\frac{1}{\rho} \sum_{n \geq 0}\left(1-\frac{S}{\rho}\right)^{n} .
$$

Let $\mathbb{C}_{\text {rat }}\langle\langle\mathbb{x}\rangle\rangle$ denote the algebra of rational series; the smallest subalgebra of $\mathbb{C}\langle\langle\mathbb{x}\rangle\rangle$ containing $\mathbb{C}\langle\mathbb{x}\rangle$ such that if $S \in \mathbb{C}_{\text {rat }}\langle\langle\mathbb{x}\rangle\rangle$ and $S^{-1}$ exists, then $S^{-1} \in \mathbb{C}_{\text {rat }}\langle\langle\mathbb{x}\rangle\rangle$.

2.1. Free analysis. We give basic definitions and a few results in free analysis that will be used throughout the paper.

A free polynomial is a noncommutative polynomial evaluated on tuples of matrices that preserves the structure of free sets. A free set $\Gamma=(\Gamma[n])_{n=1}^{\infty} \subset M(\mathbb{C})^{g}=$ $\left(M_{n}(\mathbb{C})^{g}\right)_{n=1}^{\infty}$ is a graded set of tuples of matrices that is closed under direct sums and conjugation by similarities. That is, if $X \in \Gamma[n], Y \in \Gamma[m]$ and $S \in \mathrm{GL}_{n}(\mathbb{C})$ then

(i) $X \oplus Y \in \Gamma[n+m]$;

(ii) $S^{-1} X S \in \Gamma[n]$,

where

$$
X \oplus Y=\left(X_{1}, \ldots, X_{g}\right) \oplus\left(Y_{1}, \ldots, Y_{g}\right)=\left(X_{1} \oplus Y_{1}, \ldots, X_{g} \oplus Y_{g}\right)
$$

and

$$
S^{-1} X S=\left(S^{-1} X_{1} S, \ldots, S^{-1} X_{g} S\right) .
$$

Let $U \subset M(\mathbb{C})^{g}$ be a free set. A free map (or free function) $f$ from $U$ into $M(\mathbb{C})$ is a sequence of functions $f[n]: U[n] \rightarrow M_{n}(\mathbb{C})$ that respects the free structure of $U$; 
$f(X \oplus Y)=f(X) \oplus f(Y)$ and $f\left(S^{-1} X S\right)=S^{-1} f(X) S$. The notion of a free map extends easily to vector-valued functions $f: U \rightarrow M(\mathbb{C})^{h}$, matrix-valued functions $f: U \rightarrow M_{d}\left(M(\mathbb{C})^{g}\right)$ and even operator-valued functions. If $f_{i}: U \rightarrow M(\mathbb{C})$ is a free map for $1 \leq i \leq h$ then we say the tuple $f=\left(f_{1}, \ldots, f_{h}\right)$ is a free mapping and write $f: U \rightarrow M(\mathbb{C})^{h}$.

Suppose $U \subset M(\mathbb{C})^{g}$ is a free set and each $U[n]$ is open (as a subset of $M_{n}(\mathbb{C})^{g}$ ). A free map $f: U \rightarrow M(\mathbb{C})$ is continuous if each $f[n]$ is continuous, and is (free) analytic if each $f[n]$ is analytic. As shown in [KVV14], a free function that is continuous is also free analytic (see also [HKM11]).

As one would hope, there are indeed connections between free analytic functions and formal power series. In fact, a formal power series with a positive radius of convergence determines a free analytic function and with a small degree of local boundedness we get the converse (see [HKM12]).

Given a positive integer $d$ let $f \in M_{d}(\mathbb{C})^{h}\langle\langle\mathbb{x}\rangle\rangle$, that is

$$
f=\sum_{m=0}^{\infty} \sum_{\substack{w \in\langle x\rangle \\|w|=m}} c_{w} w,
$$

where $c_{w} \in M_{d}(\mathbb{C})^{h}$. For $X \in M_{n}(\mathbb{C})^{g}$ and $w=x_{i_{1}} \ldots x_{i_{k}} \in\langle\mathbb{x}\rangle$, we say $w(X)=$ $X^{w}=X_{i_{1}} \ldots X_{i_{k}}$. We define the evaluation of $f$ at $X$ by

$$
f(X)=\sum_{m=0}^{\infty} \sum_{\substack{w \in\langle\mathbb{x}\rangle \\|w|=m}} c_{w} \otimes X^{w},
$$

provided this series converges.

\section{JACOBIAN MATRICES AND FREE ANALYTIC FUnCTIONS}

Fix $g \in \mathbb{Z}^{+}$and set $\boldsymbol{x}=\left(x_{1}, \ldots, x_{g}\right) \in\langle\mathbb{x}\rangle^{g}$, with $\boldsymbol{x}$ considered as a row vector. For $h \in \mathbb{Z}^{+}$and $1 \leq i \leq h$, let $\phi_{i} \in \mathbb{C}\langle\langle\mathbb{x}\rangle\rangle$ and $\phi=\left(\phi_{1}, \ldots, \phi_{h}\right) \in(\mathbb{C}\langle\langle\mathbb{x}\rangle\rangle)^{h}$. Alternatively, we can view $\phi$ as an element of $\mathbb{C}^{h}\langle\langle\mathbf{x}\rangle\rangle$, the set of formal power series with coefficients in $\mathbb{C}^{h}$.

Let $h, k \in \mathbb{Z}^{+}$. Suppose $\mathbb{y}=\left\{y_{1}, \ldots, y_{h}\right\}$ and $\mathbb{Z}=\left\{z_{1}, \ldots, z_{k}\right\}$ are sets of freely noncommuting indeterminates and suppose $\phi$ has no constant term, that is, $\phi \in\left(\mathbb{C}\langle\mathbb{x}\rangle_{+}\right)^{h}$. In this case, we may view $\phi$ from a much more algebraic perspective; $\phi: \mathbb{C}\left\langle\langle\mathrm{y}\rangle \rightarrow \mathbb{C}\langle\langle\mathrm{x}\rangle\rangle\right.$ is a continuous homomorphism defined by $y_{i} \mapsto \phi_{i}$.

3.1. The Jacobian matrix of a formal power series. We define the (left) noncommutative Jacobian matrix of a formal power series, a central object of study throughout the paper. A treatment of the noncommutative Jacobian matrix can be found in [Reu92].

Definition 3.1. Let $f \in \mathbb{C}\langle\langle\mathbb{x}\rangle\rangle$ with $f=\sum_{w \in\langle x\rangle} f_{w} w$. For $1 \leq i \leq g$, define $S_{x_{i}}^{*}: \mathbb{C}\langle\langle\mathrm{x}\rangle\rangle \rightarrow \mathbb{C}\langle\langle\mathrm{x}\rangle\rangle$ by

$$
S_{x_{i}}^{*} f=\sum_{w \in\langle x\rangle} f_{x_{i} w} w
$$

In other words, $S_{x_{i}}^{*}$ is the adjoint of the operator of left multiplication by $x_{i}$. 
Let $h \in \mathbb{Z}^{+}$and take $\left.\phi \in(\mathbb{C}\langle\mathbb{x}\rangle\rangle\right)^{h}$, seen as a row vector of formal power series. The $g \times h$ matrix over $\mathbb{C}\langle\langle\mathbf{x}\rangle\rangle$ defined by

$$
J_{\phi}=\left(S_{x_{j}}^{*} \phi_{i}\right)_{i, j=1}^{g, h}
$$

is the (left) Jacobian matrix of $\phi$. In particular, if $\phi$ has constant term $\phi_{\mathbb{1}}=$ $\left(\phi_{\mathbb{1}}^{1} \mathbb{1}, \ldots, \phi_{\mathbb{1}}^{h} \mathbb{1}\right)$, then

$$
\phi=\phi_{\mathbb{1}}+\boldsymbol{x} J_{\phi},
$$

where $\boldsymbol{x} J_{\phi}$ is the standard product of a row vector and a matrix. This representation of $\phi$ is unique.

Remark 3.2. Let $\phi \in\left(\mathbb{C}\left\langle\langle\mathbb{x}\rangle_{+}\right)^{h}\right.$ and define the homomorphism $\alpha: \mathbb{C}\langle\langle\mathbb{y}\rangle \rightarrow \mathbb{C}\langle\langle\mathbb{x}\rangle\rangle$ by $\alpha\left(y_{i}\right)=\phi_{i}$. Defining $J_{\alpha}=J_{\phi}$ yields the Jacobian matrix encountered in [Reu92].

It is evident that every formal power series has a Jacobian matrix and if $\alpha, \beta \in$ $(\mathbb{C}\langle\mathbb{x}\rangle\rangle)^{h}$ have the same Jacobian matrix then $\alpha-\beta \in \mathbb{C}^{h}$.

Remark 3.3. If $\phi: \mathbb{C}\langle\langle\mathrm{y}\rangle\rangle \rightarrow \mathbb{C}\langle\langle\mathbb{x}\rangle\rangle$ and $\psi: \mathbb{C}\langle\langle\mathbb{Z}\rangle\rangle \rightarrow \mathbb{C}\langle\langle\mathrm{y}\rangle\rangle$ are continuous homomorphisms, then certainly $\phi \circ \psi: \mathbb{C}\langle\mathbb{Z}\rangle \rightarrow \mathbb{C}\langle\langle\mathbb{x}\rangle\rangle$ is a continuous homomorphism. As tuples of formal power series this says that $\psi(\phi(\boldsymbol{x})) \in\left(\mathbb{C}\left\langle\langle\mathbb{x}\rangle_{+}\right)^{k}\right.$. This aligns with the fact that $\psi(\phi(\boldsymbol{x}))$ is defined as long as $\phi$ has a zero constant term.

For any $A \in M_{k \times n}\left(\mathbb{C}\langle\langle y\rangle)\right.$ and $\phi \in(\mathbb{C}\langle\langle\mathbb{x}\rangle\rangle)^{h}, A=A(\boldsymbol{y})$ and $A(\phi(\boldsymbol{x})) \in$ $M_{k \times n}(\mathbb{C}\langle\langle\mathbb{x}\rangle\rangle)$, where $A(\phi(\boldsymbol{x}))$ is the result of applying the homomorphism $y_{i} \mapsto \phi_{i}$ to each entry of $A$. Thus, if $B \in M_{n \times m}(\mathbb{C}\langle\langle y\rangle)$ then $A(\phi(\boldsymbol{x})) B(\phi(\boldsymbol{x}))=A B(\phi(\boldsymbol{x})) \in$ $M_{k \times m}\left(\mathbb{C}\langle\langle\mathbb{x}\rangle)\right.$. In particular, if $C \in M_{n}(\mathbb{C}\langle\langle\mathrm{y}\rangle)$ is an invertible matrix then $C^{-1}=C(\boldsymbol{y})^{-1}$ and $C^{-1}(\phi(\boldsymbol{x}))=C(\phi(\boldsymbol{x}))^{-1}$.

Proposition 3.4. If $\phi \in\left(\mathbb{C}\left\langle\langle\mathbb{x}\rangle_{+}\right)^{h}\right.$ and $\psi \in\left(\mathbb{C}\left\langle\langle y\rangle_{+}\right)^{k}\right.$ then

$$
J_{\psi \circ \phi}(\boldsymbol{x})=J_{\phi}(\boldsymbol{x}) J_{\psi}(\phi(\boldsymbol{x})) \in M_{g \times k}(\mathbb{C}\langle\langle\mathbb{x}\rangle) .
$$

Proof. Observe $\psi \circ \phi \in(\mathbb{C}\langle\langle\mathbb{x}\rangle\rangle)^{k}$. Define $\alpha: \mathbb{C}\langle\langle\mathrm{y}\rangle\rangle \rightarrow \mathbb{C}\langle\langle\mathbb{x}\rangle\rangle$ and $\beta: \mathbb{C}\langle\langle\mathbb{Z}\rangle \rightarrow \mathbb{C}\langle\langle\mathrm{y}\rangle\rangle$ by $\alpha\left(y_{i}\right)=\phi_{i}$ and $\beta\left(z_{j}\right)=\psi_{j}$. Thus, $\alpha \circ \beta: \mathbb{C}\langle\langle\mathbb{Z}\rangle\rangle \rightarrow \mathbb{C}\langle\langle\mathbb{x}\rangle\rangle$ with $\alpha \circ \beta\left(z_{i}\right)=(\psi \circ \phi)_{i}$. By Proposition 1.2 in [Reu92], $J_{\psi \circ \phi}=J_{\phi}(\boldsymbol{x}) J_{\psi}(\phi(\boldsymbol{x})) \in M_{g \times k}(\mathbb{C}\langle\langle\mathbb{x}\rangle\rangle)$.

Corollary 3.5. Suppose $p, q \in\left(\mathbb{C}\left\langle\langle\mathbb{x}\rangle_{+}\right)^{g}\right.$ have Jacobian matrices $J_{p}$ and $J_{q}$, respectively. The series $p$ and $q$ are compositional inverses if and only if $J_{p}(q(\boldsymbol{x}))=$ $J_{q}(\boldsymbol{x})^{-1}$ and $J_{q}(p(\boldsymbol{x}))=J_{p}(\boldsymbol{x})^{-1}$.

Proof. Suppose $p$ and $q$ are compositional inverses. Hence $p(q(\boldsymbol{x}))=\boldsymbol{x}$ and $q(p(\boldsymbol{x}))=$ $\boldsymbol{x}$. Applying Proposition 3.4,

$$
J_{q}(\boldsymbol{x}) J_{p}(q(\boldsymbol{x}))=J_{p \circ q}(\boldsymbol{x})=I_{g}
$$

and

$$
J_{p}(\boldsymbol{x}) J_{q}(p(\boldsymbol{x}))=J_{q \circ p}(\boldsymbol{x})=I_{g} .
$$

Thus $J_{p}(q(\boldsymbol{x}))=J_{q}(\boldsymbol{x})^{-1}$ and $J_{q}(p(\boldsymbol{x}))=J_{p}(\boldsymbol{x})^{-1}$.

Now suppose $J_{p}(q(\boldsymbol{x}))=J_{q}(\boldsymbol{x})^{-1}$ and $J_{q}(p(\boldsymbol{x}))=J_{p}(\boldsymbol{x})^{-1}$. Observe

$$
p(q(\boldsymbol{x}))=\boldsymbol{x} J_{p \circ q}(\boldsymbol{x})=\boldsymbol{x} J_{q}(\boldsymbol{x}) J_{p}(q(\boldsymbol{x}))=\boldsymbol{x} I_{g}=\boldsymbol{x},
$$

and

$$
q(p(\boldsymbol{x}))=\boldsymbol{x} J_{q \circ p}(\boldsymbol{x})=\boldsymbol{x} J_{p}(\boldsymbol{x}) J_{q}(p(\boldsymbol{x}))=\boldsymbol{x} I_{g}=\boldsymbol{x} .
$$

Therefore, $p$ and $q$ are compositional inverses. 
The invertibility of the Jacobian matrix is reminiscent of the inverse function theorem. Indeed, if $p \in\left(\mathbb{C}\langle\langle\mathbb{x}\rangle\rangle_{+}\right)^{g}$ is a free function, then $p$ is locally invertible at 0 if and only if $J_{p}$ is invertible at 0 .

It should be noted that $J_{\alpha^{-1}}(\boldsymbol{x})=J_{\alpha}^{-1}\left(\alpha^{-1}(\boldsymbol{x})\right)$, hence we cannot use $J_{\alpha}$ to directly compute $\alpha^{-1}$ without already knowing the explicit form of $\alpha^{-1}$. However, $J_{\alpha}^{-1}$ is a local approximation of $J_{\alpha^{-1}}$, implying we may be able to construct $\alpha^{-1}$ from successive approximations. This leads us directly to subsection 3.2.

3.2. Auxiliary inverses and compositional inverses. The main result in this subsection, Proposition 3.12, tells us that if $p \in(\mathbb{C}\langle\langle\mathbf{x}\rangle\rangle)^{g}$, such that $J_{p}$ is invertible, then $p^{-1}$ is the limit of a sequence of polynomials constructed from $J_{p}^{-1}$.

Definition 3.6. Suppose $h \in \mathbb{Z}^{+}$and $\mathbb{Z}=\left\{z_{1}, \ldots, z_{h}\right\}$ is a set of freely noncommuting indeterminates. For any $w \in\langle\mathbb{x} \cup \mathbb{Z}\rangle$ define $|w|_{z}$ to be the number of $z$-terms appearing in $w$ and define $|w|_{x}$ to be the number of $x$-terms appearing in $w$. In particular, $|w|=|w|_{x}+|w|_{z}$.

Let $\ell \in \mathbb{Z}^{+}$and $\alpha \in \mathbb{C}^{\ell}\left\langle\langle\mathbb{x} \cup \mathbb{Z}\rangle_{+} \cong\left(\mathbb{C}\left\langle\langle\mathbb{\mathbb { X }} \cup \mathbb{Z}\rangle_{+}\right)^{\ell}\right.\right.$ with $\alpha=\sum_{w \in\langle\mathbb{x} \cup \mathbb{Z}\rangle} a_{w} w$. Define

$$
\mathbb{d}_{z}(\alpha)=\inf \left\{|w|:|w|_{z}>0 \text { and } a_{w} \neq 0\right\} .
$$

Note if $\alpha$ has no $z$-terms then $\mathbb{d}_{z}(\alpha)=\infty$.

We will consistently write a formal power series $\alpha \in\left(\mathbb{C}\langle\langle\mathbb{x} \cup \mathbb{Z}\rangle)^{\ell}\right.$ as $\alpha(\boldsymbol{x})[\boldsymbol{z}]$ rather than $\alpha(\boldsymbol{x}, \boldsymbol{z})$. This convention is simply a preference based on aligning our notation with the notation we use for free derivatives. Thus, if $\beta \in\left(\mathbb{C}\langle\mathbb{\mathbb { x }} \cup \mathbb{Z}\rangle_{+}\right)^{h}$ then $\alpha(\boldsymbol{x})[\beta(\boldsymbol{x})[\boldsymbol{z}]]$ written another way is $\alpha(\boldsymbol{x}, \beta(\boldsymbol{x}, \boldsymbol{z}))$.

Lemma 3.7. Let $\alpha, \beta \in \mathbb{C}^{\ell}\left\langle\langle\mathbb{x} \cup \mathbb{Z}\rangle_{+}\right.$and $\gamma \in \mathbb{C}^{h}\langle\langle\mathbb{x} \cup \mathbb{Z}\rangle\rangle$ with $\alpha=\sum_{w \in\langle\mathbb{x} \cup \mathbb{Z}\rangle} a_{w} w$, $\beta=\sum_{w \in\langle\mathbb{x} \cup \mathbb{Z}\rangle} b_{w} w$, and $\gamma=\sum_{w \in\langle\mathbb{x} \cup \mathbb{Z}\rangle} c_{w} w$. We have the following,

(i) $\mathbb{d}_{z}(\alpha+\beta) \geq \min \left\{\mathbb{d}_{z}(\alpha), \mathbb{d}_{z}(\beta)\right\}$;

(ii) $\mathbb{d}_{z}(\alpha \beta) \geq \min \left\{\mathbb{d}_{z}(\alpha), \mathbb{d}_{z}(\beta)\right\}$ and in particular, if $\mathbb{d}_{z}(\alpha)$ and $\mathbb{d}_{z}(\beta)$ are not both infinite, then $\mathbb{d}_{z}(\alpha \beta)>\min \left\{\mathbb{d}_{z}(\alpha), \mathbb{d}_{z}(\beta)\right\}$;

(iii) if $\mathbb{d}_{z}(\alpha)>1$ and $\mathbb{d}_{z}(\gamma)<\infty$ then $\mathbb{d}_{z}(\gamma)<\mathbb{d}_{z}(\alpha(\boldsymbol{x})[\gamma(\boldsymbol{x})[\boldsymbol{z}]])$.

Proof. Since $a_{w}+b_{w}$ is nonzero only if at least one of $a_{w}$ or $b_{w}$ is nonzero, we automatically have $\mathbb{d}_{z}(\alpha+\beta) \geq \min \left\{\mathbb{d}_{z}(\alpha), \mathbb{d}_{z}(\beta)\right\}$. Thus we have proven $(i)$.

To prove (ii), first suppose $\mathbb{d}_{z}(\alpha), \mathbb{d}_{z}(\beta)=\infty$, i.e. neither has a $z$-term. It follows that their product, $\alpha \beta$ has no $z$-terms and thus $\mathbb{d}_{z}(\alpha \beta)=\infty$.

Now suppose $\mathbb{d}_{z}(\alpha)$ is finite. If $\mathbb{d}_{z}(\alpha \beta)$ is infinite then $\mathbb{d}_{z}(\alpha \beta)>\mathbb{d}_{z}(\alpha) \geq$ $\min \left\{\mathbb{d}_{z}(\alpha), \mathbb{d}_{z}(\beta)\right\}$ and we are done. Finally, suppose $\mathbb{d}_{z}(\alpha \beta)<\infty$ and let $\alpha \beta=$ $\sum_{w \in\langle\mathbb{x} \cup \mathbb{Z}\rangle} c_{w} w$. Let $w$ be a monomial with $|w|_{z}>0,|w|=\mathbb{d}_{z}(\alpha \beta)$ and $c_{w} \neq 0$. There exist monomials $u, v$ such that $a_{u} b_{v} \neq 0$ and $u v=w$. Recall $\alpha$ and $\beta$ have no constant terms so $|u|,|v|>0$. Since $|w|_{z}>0$ we may assume $|u|_{z}>0$, hence $|u| \geq \mathbb{d}_{z}(\alpha)$ and

$$
\mathbb{d}_{z}(\alpha \beta)=|w|=|u|+|v| \geq \mathbb{d}_{z}(\alpha)+|v|>\mathbb{d}_{z}(\alpha) \geq \min \left\{\mathbb{d}_{z}(\alpha), \mathbb{d}_{z}(\beta)\right\} .
$$

Thus, $\mathbb{d}_{z}(\alpha \beta)>\min \left\{\mathbb{d}_{z}(\alpha), \mathbb{d}_{z}(\beta)\right\}$ and item (ii) is done.

To prove item (iii), suppose $\mathbb{d}_{z}(\alpha)>1$ and $\mathbb{d}_{z}(\gamma)<\infty$. Set

$$
W=\left\{w \in\langle\mathbb{x} \cup \mathbb{Z}\rangle:|w|_{z}>0, a_{w} \neq 0\right\}
$$


and note if $W$ is empty then $\mathbb{d}_{z}(\alpha(\boldsymbol{x})[\gamma(\boldsymbol{x})[\boldsymbol{z}]])=\infty>\mathbb{d}_{z}(\gamma)$. Suppose $W$ is nonempty. Writing

$$
\alpha(\boldsymbol{x})[\gamma(\boldsymbol{x})]=\sum_{w \in\langle\boldsymbol{x} \cup \mathbb{Z}\rangle} a_{w} w(\boldsymbol{x})[\gamma(\boldsymbol{x})[\boldsymbol{z}]]
$$

and applying item (i) yields

$$
\mathbb{d}_{z}(\alpha(\boldsymbol{x})[\gamma(\boldsymbol{x})[\boldsymbol{z}]]) \geq \min \left\{\mathbb{d}_{z}\left(a_{w} w(\boldsymbol{x})[\gamma(\boldsymbol{x})[\boldsymbol{z}]]\right): w \in W\right\} .
$$

Suppose there is a $w \in W$ such that $\mathbb{d}_{z}\left(a_{w} w(\boldsymbol{x})[\gamma(\boldsymbol{x})[\boldsymbol{z}]]\right)<\infty$ (if not, then $\mathbb{d}_{z}(\alpha(\boldsymbol{x})[\gamma(\boldsymbol{x})[\boldsymbol{z}]])=\infty$ and we are done). We know $|w| \geq 2$ since $w \in W$ and $\mathbb{d}_{z}(\alpha) \geq 2$. Hence $w(\boldsymbol{x})[\gamma(\boldsymbol{x})[\boldsymbol{z}]]$ is a finite product of $x_{i}$ and $\gamma_{i}(\boldsymbol{x})[\boldsymbol{z}]$ terms. Thus, for $w \in W, \mathbb{d}_{z}\left(a_{w} w(\boldsymbol{x})[\gamma(\boldsymbol{x})[\boldsymbol{z}]]\right)>\mathbb{d}_{z}(\gamma)$, and therefore $\mathbb{d}_{z}(\alpha(\boldsymbol{x})[\gamma(\boldsymbol{x})[\boldsymbol{z}]])>$ $\mathbb{d}_{z}(\gamma)$.

Lemma 3.8. Suppose $\mathrm{a} \in\left(\mathbb{C}\left\langle\langle\mathbb{x} \cup \mathbb{Z}\rangle_{+}\right)^{h}\right.$ and define

$$
\mathrm{a}^{\circ k}(\boldsymbol{x})[\boldsymbol{z}]=\mathrm{a}(\boldsymbol{x})\left[\mathrm{a}^{\circ k-1}(\boldsymbol{x})[\boldsymbol{z}]\right]
$$

for $k>1$ and $\mathfrak{a}^{\circ 1}(\boldsymbol{x})[\boldsymbol{z}]=\mathrm{a}(\boldsymbol{x})[\boldsymbol{z}]$. If $n, m \in \mathbb{Z}^{+}$then

$$
\mathrm{a}^{\circ n}(\boldsymbol{x})\left[\mathrm{a}^{\circ m}(\boldsymbol{x})[\boldsymbol{z}]\right]=\mathrm{a}^{\circ m}(\boldsymbol{x})\left[\mathrm{a}^{\circ n}(\boldsymbol{x})[\boldsymbol{z}]\right]=\mathrm{a}^{\circ n+m}(\boldsymbol{x})[\boldsymbol{z}] .
$$

Proof. We first prove

$$
\mathrm{a}^{\circ n+1}(\boldsymbol{x})[\boldsymbol{z}]=\mathrm{a}(\boldsymbol{x})\left[\mathrm{a}^{\circ n}(\boldsymbol{x})[\boldsymbol{z}]\right]=\mathrm{a}^{\circ n}(\boldsymbol{x})[\mathrm{a}(\boldsymbol{x})[\boldsymbol{z}]]
$$

via induction. The base case is from the definition, so suppose (3.2) holds for $n$ and consider

$$
\begin{aligned}
\mathrm{a}^{\circ n+2}(\boldsymbol{x})[\boldsymbol{z}] & =\mathrm{a}(\boldsymbol{x})\left[\mathrm{a}^{\circ n+1}(\boldsymbol{x})[\boldsymbol{z}]\right]=\mathrm{a}(\boldsymbol{x})\left[\mathrm{a}^{\circ n}(\boldsymbol{x})[\mathrm{a}(\boldsymbol{x})[\boldsymbol{z}]]\right] \\
& =\mathrm{a}^{\circ n+1}(\boldsymbol{x})[\mathrm{a}(\boldsymbol{x})[\boldsymbol{z}]] .
\end{aligned}
$$

Thus (3.2) holds in general.

Now, take any $n, m \in \mathbb{Z}^{+}$and consider $\boldsymbol{a}^{\circ n}(\boldsymbol{x})\left[\boldsymbol{a}^{\circ m}(\boldsymbol{x})[\boldsymbol{z}]\right]$. Applying (3.2) $m$ times yields $\mathrm{a}^{\circ n}(\boldsymbol{x})\left[\mathrm{a}^{\circ m}(\boldsymbol{x})[\boldsymbol{z}]\right]=\mathrm{a}^{\circ n+m}(\boldsymbol{x})[\boldsymbol{z}]$, while applying (3.2) $n$ times gives $\mathrm{a}^{\circ n+m}(\boldsymbol{x})[\boldsymbol{z}]=\mathrm{a}^{\circ m}(\boldsymbol{x})\left[\mathrm{a}^{\circ n}(\boldsymbol{x})[\boldsymbol{z}]\right]$.

Suppose $\mathbf{a} \in\left(\mathbb{C}\left\langle\langle\mathbb{x} \cup \mathbb{Z}\rangle_{+}\right)^{h}\right.$. For each $k \geq 1$ set $\mathbb{d}_{a}^{k}=\mathbb{d}_{z}\left(\mathrm{a}^{\circ k}\right)$ and $\mathbf{a}^{\circ k}(\boldsymbol{x})[\boldsymbol{z}]=$ $\sum_{w \in\langle\mathbb{x} \cup \mathbb{Z}\rangle} c_{w}^{k} w$. We define

$$
\mathfrak{a}^{k}(\boldsymbol{x})=\sum_{\substack{w \in\langle\mathbb{X}\rangle \\|w|<\mathbb{d}_{a}^{k}}} c_{w}^{k} w \quad \text { and } \quad \alpha^{k}(\boldsymbol{x})[\boldsymbol{z}]=\sum_{\substack{w \in\langle\mathbb{x} \cup \mathbb{Z}\rangle \\|w| \geq \mathbb{d}_{a}^{k}}} c_{w}^{k} w,
$$

and observe

$$
\mathrm{a}^{\circ k}(\boldsymbol{x})[\boldsymbol{z}]=\mathfrak{a}^{k}(\boldsymbol{x})+\alpha^{k}(\boldsymbol{x})[\boldsymbol{z}] .
$$

Lemma 3.9. Suppose $\mathfrak{a} \in\left(\mathbb{C}\left\langle\langle\mathbb{x} \cup \mathbb{Z}\rangle_{+}\right)^{h}\right.$ and $\mathbb{d}_{z}(\mathfrak{a})>1$. The sequences $\left(\mathfrak{a}^{k}\right)$ and $\mathbb{d}_{a}^{k}$ have the following properties.

(i) $\mathbb{d}_{a}^{k}$ is either strictly increasing with $k$, or there is an $N$ such that if $k<N$ then $\mathbb{d}_{a}^{k}<\mathbb{d}_{a}^{k+1}$ and if $k \geq N$ then $\mathbb{d}_{a}^{k}=\infty$;

(ii) $\mathbb{d}_{a}^{k}>k$ for all $k$;

(iii) If $n \geq k$ then the coefficients of $\mathrm{a}^{\circ k}$ and $\mathrm{a}^{\circ n}$ agree on monomials of length less than $\mathbb{d}_{a}^{k}$ and, in particular, the coefficients of $\mathfrak{a}^{k}$ and $\mathfrak{a}^{n}$ agree on monomials of length less than $\mathbb{d}_{a}^{k}$; 
(iv) $\left(\mathfrak{a}^{k}\right)$ is a convergent sequence (in the topology of $\left.\left.(\mathbb{C}\langle\mathbf{x}\rangle\rangle\right)^{g}\right)$ and letting $a=\lim _{k \rightarrow \infty} \mathfrak{a}^{k}$ we have $a(\boldsymbol{x})=\mathbf{a}(\boldsymbol{x})[a(\boldsymbol{x})]$. Moreover, $a$ is the unique function such that $a(\boldsymbol{x})=\mathbf{a}(\boldsymbol{x})[a(\boldsymbol{x})]$.

Proof. If $\mathbb{d}_{a}^{k+1}<\infty$ then there is a monomial $w$ appearing in $\mathrm{a}^{\circ k+1}$ with $|w|_{z}>0$. However, since $\mathrm{a}^{\circ k+1}(\boldsymbol{x})[\boldsymbol{z}]=\mathrm{a}(\boldsymbol{x})\left[\mathrm{a}^{\circ k}(\boldsymbol{x})[\boldsymbol{z}]\right]$, Lemma 3.7(iii) tells us exactly $\mathbb{d}_{a}^{k+1}>\mathbb{d}_{z}^{k}$.

Suppose $\mathbb{d}_{a}^{n}=\infty$ for some $n$ and set $N=\min \left\{k: \mathbb{d}_{a}^{k}=\infty\right\} \geq 1$. We note $\mathbb{d}_{a}^{N-1}<\infty=\mathbb{d}_{a}^{N}$ and if $k<N-1$ then $\mathbb{d}_{a}^{k}<\mathbb{d}_{a}^{k+1}<\infty$. For any $k \geq N$,

$$
\mathrm{a}^{\circ k}(\boldsymbol{x})[\boldsymbol{z}]=\mathrm{a}^{\circ N}(\boldsymbol{x})\left[\mathrm{a}^{\circ k-N}(\boldsymbol{x})[\boldsymbol{z}]\right]
$$

by Lemma 3.8. Since there are no $z$-terms appearing in $a^{\circ N}$,

$$
\mathrm{a}^{\circ k}(\boldsymbol{x})[\boldsymbol{z}]=\mathrm{a}^{\circ N}(\boldsymbol{x})\left[\mathrm{a}^{\circ k-N}(\boldsymbol{x})[\boldsymbol{z}]\right]=\mathrm{a}^{\circ N}(\boldsymbol{x})[\boldsymbol{z}]
$$

Hence $\mathbb{d}_{a}^{k}=\mathbb{d}_{a}^{N}=\infty$ and item (i) is proved.

By item (i) we know $\mathbb{d}_{a}^{k+1} \geq \mathbb{d}_{a}^{k}+1$ for all $k$. Since $\mathbb{d}_{a}^{1}>1$ by hypothesis, we see that $\mathbb{d}_{a}^{k} \geq \mathbb{d}_{a}^{1}+k-1>k$, thus item (ii) is proved.

First, recall from (3.4) that $\mathfrak{a}^{\circ k}(\boldsymbol{x})[\boldsymbol{z}]=\mathfrak{a}^{k}(\boldsymbol{x})+\alpha^{k}(\boldsymbol{x})[\boldsymbol{z}]$. If $\alpha^{k}=0$ then $\mathbb{d}_{a}^{k}=\infty$ and $\mathfrak{a}^{\circ k}(\boldsymbol{x})[\mathfrak{a}(\boldsymbol{x})[\boldsymbol{z}]]=\mathfrak{a}^{\circ k}(\boldsymbol{x})[\boldsymbol{z}]$, hence the coefficients of $\mathfrak{a}^{k}$ and $\mathfrak{a}^{k+1}$ agree up to $\mathbb{d}_{a}^{k}$, i.e. $\mathfrak{a}^{k}=\mathfrak{a}^{k+1}$.

If $\alpha^{k} \neq 0$ then the minimum length of a monomial appearing in $\alpha^{k}(\boldsymbol{x})[\mathrm{a}(\boldsymbol{x})[\boldsymbol{z}]]$ is at least $\mathbb{d}_{a}^{k}$ since the minimum length of a monomial appearing in $\alpha^{k}(\boldsymbol{x})[\boldsymbol{z}]$ is $\mathbb{d}_{a}^{k}$. Hence, the coefficients of $\boldsymbol{a}^{\circ k+1}(\boldsymbol{x})[\boldsymbol{z}]$ and $\mathrm{a}^{\circ k}(\boldsymbol{x})[\boldsymbol{z}]$ agree on monomials of length less than $\mathbb{d}_{a}^{k}$, and in particular, the coefficients of $\mathfrak{a}^{k}$ and $\mathfrak{a}^{k+1}$ agree on monomials of length less than $\mathbb{d}_{a}^{k}$. Hence, with iteration, if $n \geq k$ then the coefficients of $\mathrm{a}^{\circ k}$, $\mathrm{a}^{\circ n}$, $\mathfrak{a}^{k}$ and $\mathfrak{a}^{n}$ agree on monomials of length less than $\mathbb{d}_{a}^{k}$. Thus, item (iii) is proved.

Finally, to prove item (iv), we observe $d\left(\mathfrak{a}^{n}, \mathfrak{a}^{m}\right) \leq 2^{-\min \{n, m\}}$ (recall $d$ is the metric on formal power series), hence $\left(\mathfrak{a}^{k}\right)$ is a Cauchy sequence and thus converges. Set $a=\lim _{k \rightarrow \infty} \mathfrak{a}^{k}$.

Let $n \in \mathbb{Z}^{+}$be given and note $\mathbb{d}_{a}^{n}>n$, by item (ii). By item (iii), the coefficients of $a, \mathrm{a}^{\circ n}$ and $\mathfrak{a}^{n}$ agree on monomials of length less than $\mathbb{d}_{a}^{n}$. Hence, the coefficients of $\mathfrak{a}^{n}(\boldsymbol{x}), \mathfrak{a}(\boldsymbol{x})\left[\mathfrak{a}^{n}(\boldsymbol{x})\right]$ and $\mathfrak{a}^{\circ n}(\boldsymbol{x})[\boldsymbol{z}]$ all agree on monomials of length less than $n$. Consequently, the coefficients of $a(\boldsymbol{x})$ and $\boldsymbol{a}(\boldsymbol{x})[a(\boldsymbol{x})]$ must agree on all monomials of length less than $n$. Thus $a(\boldsymbol{x})=\mathbf{a}(\boldsymbol{x})[a(\boldsymbol{x})]$.

If $\hat{a}$ is any formal power series mapping such that $\mathbf{a}(\boldsymbol{x})[\hat{a}(\boldsymbol{x})]=\hat{a}(\boldsymbol{x})$ then $\mathrm{a}^{\circ n}(\boldsymbol{x})[\hat{a}(\boldsymbol{x})]=\hat{a}(\boldsymbol{x})$ for all $n \geq 1$. However, this implies that the coefficients of $a$ and $\hat{a}$ agree on monomials of length less than $n$, for all $n$. Thus, $\hat{a}=a$.

In order to connect items (iii) and (iv) to other ideas from analysis we define a partial ordering on $\mathbb{C}\langle\langle\mathbb{x}\rangle\rangle$. If $\alpha=\sum_{w} a_{w} w$ and $\beta=\sum_{w} b_{w} w$, then we say $\alpha \leq \beta$ if $a_{w}=0$ whenever $b_{w}=0$ and $a_{w}=b_{w}$ whenever $a_{w} \neq 0$. Thus, Lemma 3.9 says $\left(\mathfrak{a}^{n}\right)$ is an increasing sequence of polynomials with $a$ as its unique limit.

Under the correct reformulation, Lemma 3.9 is actually an implicit function theorem. In Section 4 we fully define the free derivative of a formal power series, allowing us to easily state and prove Theorem 4.8, the implicit function theorem for free formal power series.

Although the definitions and results in Lemma 3.9 are valid when $g \neq h$, when applying these ideas to Jacobian matrices we often assume $g=h$. 
Definition 3.10. Suppose $p \in\left(\mathbb{C}\left\langle\langle\mathbb{x}\rangle_{+}\right)^{g}\right.$ has a Jacobian matrix $J_{p} \in M_{g}(\mathbb{C}\langle\langle\mathbb{x}\rangle)$ such that $J_{p}^{-1} \in M_{g}(\mathbb{C}\langle\langle\mathbb{x}\rangle\rangle)$. Define the auxiliary inverse of $p$ to be $\mathbb{q}(\boldsymbol{x})[\boldsymbol{z}]=$ $\boldsymbol{x} J_{p}^{-1}(\boldsymbol{z}) \in\left(\mathbb{C}\left\langle\langle\mathbb{x} \cup \mathbb{Z}\rangle_{+}\right)^{g}\right.$ and recursively define the $\boldsymbol{k}^{\text {th }}$ auxiliary inverse by

$$
\mathrm{q}^{\circ k}(\boldsymbol{x})[\boldsymbol{z}]=\mathrm{qI}(\boldsymbol{x})\left[\mathrm{q}^{\circ k-1}(\boldsymbol{x})[\boldsymbol{z}]\right]
$$

where $\mathbb{q}^{\circ 1}(\boldsymbol{x})[\boldsymbol{z}]=\mathbb{I}(\boldsymbol{x})[\boldsymbol{z}]$.

The indeterminates $z_{1}, \ldots, z_{g}$, in $\mathbb{I}(\boldsymbol{x})[\boldsymbol{z}]$ are 'targets' for composition of $\mathbb{q}$ with itself. As such it is good to understand how the $z$ terms behave under the successive compositions. We imitate the setup of Lemma 3.7. For any $k \geq 1$ we write

$$
\mathrm{q}^{\circ k}(\boldsymbol{x})[\boldsymbol{z}]=\sum_{w \in\langle\mathbb{x} \cup \mathbb{Z}\rangle} \rho_{w}^{k} w
$$

where $\rho_{w}^{k} \in \mathbb{C}^{g}$, and for shorthand purposes we set $\mathbb{d}_{q}^{k}=\mathbb{d}_{z}\left(\mathbb{q}^{\circ k}\right)$. We split $\mathbb{q}^{\circ k}$ into terms with degree less than $\mathbb{d}_{q}^{k}$ and those with degree greater than or equal $\mathbb{d}_{q}^{k}$;

$$
\mathfrak{q}^{k}(\boldsymbol{x})=\sum_{\substack{w \in\langle\mathrm{x}\rangle \\|w|<\mathbb{d}_{q}^{k}}} \rho_{w}^{k} w \quad \text { and } \quad r^{k}(\boldsymbol{x})[\boldsymbol{z}]=\sum_{\substack{w \in\langle\mathbb{x} \cup \mathbb{Z}\rangle \\|w| \geq \mathbb{d}_{q}^{k}}} \rho_{w}^{k} w .
$$

Thus

$$
\mathbb{1}^{\circ k}(\boldsymbol{x})[\boldsymbol{z}]=\mathfrak{q}^{k}(\boldsymbol{x})+r^{k}(\boldsymbol{x})[\boldsymbol{z}] .
$$

Since the minimum length of any monomial appearing in $r^{k}(\boldsymbol{x})[\mathrm{q}(\boldsymbol{x})[\boldsymbol{z}]]$ is greater than $\mathbb{d}_{q}^{k}$, we have $\rho_{w}^{k}=\rho_{w}^{k+1}$ for all $|w|<\mathbb{d}_{q}^{k}$.

Remark 3.11. Since the auxiliary inverse, $\mathbb{\mathbb { I }}(\boldsymbol{x})[\boldsymbol{z}]=\boldsymbol{x} J_{p}^{-1}(\boldsymbol{z})$, we note that $\mathbb{d}_{q}^{1}=$ $\mathbb{d}_{z}(\mathfrak{q})>1$. Hence, Lemmas 3.8 and 3.9 apply to $\mathbb{q}$.

Proposition 3.12. Suppose $p$ is a free formal power series mapping without constant term. There is a free formal power series mapping $q$ (without constant term) such that $p$ and $q$ are compositional inverses if and only if $J_{p}$ has a (free formal power series) multiplicative inverse. In this case, $J_{p}(q(\boldsymbol{x}))=J_{q}^{-1}(\boldsymbol{x})$. Moreover, $q$ is the unique solution of

$$
q(\boldsymbol{x})=\boldsymbol{x} J_{p}^{-1}(q(\boldsymbol{x})) .
$$

Proof. By Corollary 3.5 we know that $p$ and $q$ are compositional inverses if and only if $J_{q}(\boldsymbol{x})=J_{p}^{-1}(q(\boldsymbol{x}))$ and $J_{p}(\boldsymbol{x})=J_{q}^{-1}(p(\boldsymbol{x}))$.

Lemma 3.9 implies there exists a unique $q \in\left(\mathbb{C}\left\langle\langle\mathbb{x}\rangle_{+}\right)^{g}\right.$ such that $\mathbb{q}(\boldsymbol{x})[q(\boldsymbol{x})]=$ $q(\boldsymbol{x})$, where $\mathbb{q}(\boldsymbol{x})[\boldsymbol{z}]=\boldsymbol{x} J_{p}^{-1}(\boldsymbol{z})$ is the auxiliary inverse of $p$. Since $q \in\left(\mathbb{C}\langle\boldsymbol{x}\rangle_{+}\right)^{g}$, we see that $J_{p}^{-1}(q(\boldsymbol{x})) \in M_{g}(\mathbb{C}\langle\langle\mathbf{x}\rangle))$ is defined and $J_{p}(q(\boldsymbol{x}))$ and $J_{p}^{-1}(q(\boldsymbol{x}))$ are inverses. Hence $q(\boldsymbol{x})=\mathbb{T}(\boldsymbol{x})[q(\boldsymbol{x})]=\boldsymbol{x} J_{p}^{-1}(q(\boldsymbol{x}))$ and

$$
p(q(\boldsymbol{x}))=q(\boldsymbol{x}) J_{p}(q(\boldsymbol{x}))=\boldsymbol{x} J_{p}^{-1}(q(\boldsymbol{x})) J_{p}(q(\boldsymbol{x}))=\boldsymbol{x} I_{g}=\boldsymbol{x} .
$$

Next, $q \in \mathbb{C}\langle\langle\mathbb{x}\rangle\rangle_{+}^{g}$ also has an auxiliary inverse, $\mathbb{p}(\boldsymbol{x})[\boldsymbol{z}]=\boldsymbol{x} J_{p}(q(\boldsymbol{z}))$. Applying Lemma 3.9 and the same argument as above we know there is a $\tilde{p} \in \mathbb{C}\langle\langle\mathbb{x}\rangle\rangle_{+}^{g}$ such that $\boldsymbol{x}=q(\tilde{p}(\boldsymbol{x}))$ and

$$
\tilde{p}(\boldsymbol{x})=\mathbb{p}(\boldsymbol{x})[\tilde{p}(\boldsymbol{x})]=\boldsymbol{x} J_{p}(q(\tilde{p}(\boldsymbol{x}))) .
$$

However, since $q(\tilde{p}(\boldsymbol{x}))=\boldsymbol{x}$,

$$
\tilde{p}(\boldsymbol{x})=\boldsymbol{x} J_{p}(q(\tilde{p}(\boldsymbol{x})))=\boldsymbol{x} J_{p}(\boldsymbol{x})=p(\boldsymbol{x}) .
$$


Thus $q(p(\boldsymbol{x}))=\boldsymbol{x}$. Therefore, $q(\boldsymbol{x})=\mathbb{q}(\boldsymbol{x})[q(\boldsymbol{x})]$ and $p$ and $q$ are compositional inverses.

We note that Proposition 3.12 does not require that $p$ corresponds to a bijective free analytic map. However, $J_{p}(0)$ and $J_{p}^{-1}(0)$ both exist, thus with an application of the free inverse function theorem (Theorem 5 in [Pas14]) we get that $p$ is locally invertible on some open free set containing the origin.

We now have conditions guaranteeing a formal power series has a compositional inverse and in fact, we have a way to calculate the inverse, or at least to approximate it.

Definition 3.13. We once again suppose $\mathbb{Z}=\left\{z_{1}, \ldots, z_{h}\right\}$, where $h$ is not necessarily equal to $g$. Suppose $\alpha \in(\mathbb{C}\langle\mathbb{x} \cup \mathbb{Z}\rangle)^{h}$. We say $\alpha$ is a proper algebraic polynomial ${ }^{1}$ if $\alpha$ has no constant terms and $\mathbb{d}_{z}(\alpha)>1$.

We say $\beta \in\left(\mathbb{C}\left\langle\langle\mathbb{x}\rangle_{+}\right)^{h}\right.$ is a solution to the proper algebraic polynomial if $\alpha(\boldsymbol{x})[\beta(\boldsymbol{x})]=\beta(\boldsymbol{x})$. Each $\beta^{i}$ is called a component of the solution.

By either Lemma 3.9 or Theorem 6.6.3 in [Sta11], every proper algebraic polynomial has a unique solution. Let $\gamma \in \mathbb{C}\langle\langle\mathbb{x}\rangle\rangle$ with constant term $c$. We say $\gamma$ is algebraic if $\gamma-c$ is a component of the solution to some proper algebraic polynomial.

Proposition 3.14. Suppose $p \in\left(\mathbb{C}\langle\mathbb{x}\rangle_{+}\right)^{g}$ and $J_{p} \in M_{g}(\mathbb{C}\langle\mathbb{x}\rangle)$ is the Jacobian matrix of $p$. If $J_{p}^{-1} \in M_{g}(\mathbb{C}\langle\mathbb{x}\rangle)$, then the compositional inverse of $p$ is algebraic.

Proof. Recall $\mathbb{q}$, the auxiliary inverse of $p$, is given by $\mathbb{q}(\boldsymbol{x})[\boldsymbol{z}]=\boldsymbol{x} J_{p}^{-1}(\boldsymbol{z})$. Proposition 3.12 tells us there is a $q \in(\mathbb{C}\langle\langle\mathbb{x}\rangle\rangle)^{g}$ such that $q$ and $p$ are compositional inverses and $\mathbb{\mathbb { I }}(\boldsymbol{x})[q(\boldsymbol{x})]=q(\boldsymbol{x})$. Observe $\mathbb{\mathbb { I }}(\boldsymbol{x})[\boldsymbol{z}]$ has no constant terms and $\mathbb{d}_{z}(\mathrm{q})>1$. Thus $\mathrm{qI}(\boldsymbol{x})[\boldsymbol{z}]$ is a proper algebraic polynomial and $q$ is the unique algebraic function satisfying $\mathbb{q}(\boldsymbol{x})[q(\boldsymbol{x})]=q(\boldsymbol{x})$.

We know every polynomial mapping is a rational mapping and Example 6.6.5 in [Sta11] shows every rational mapping is an algebraic mapping. Unfortunately, this does not help us prove a bijective free polynomial has a free polynomial inverse (at least not directly).

If $p$ is not bijective then it may still have a compositional inverse that is algebraic. The auxiliary inverse can be a polynomial even if $p$ is not injective, as Example 3.19 shows. In the case where $p$ is not injective but $\mathrm{q}$ is still a polynomial, we get a unique algebraic function $q$ so that $p(q(X))=X$ and $q(p(X))=X$ whenever these compositions are defined.

3.3. Invertibility of the Jacobian matrix. In this section we establish the following result about bijective free polynomials:

Theorem 3.17. If $p$ is a bijective polynomial mapping, then $J_{p}^{-1}$ is a polynomial (matrix).

By using the Free Grothendieck theorem, we have that every injective free polynomial has a free polynomial inverse. Hence, Theorem 3.17 is an unsurprising consequence of the chain rule. However, Theorem 3.17 is critical for the proof of the Free Grothendieck theorem and we cannot forgo its exposition.

\footnotetext{
${ }^{1}$ This definition differs from the established terminology often seen in enumerative combinatorics and automata theory. In those contexts we say a system of equations $\alpha(\boldsymbol{x})[\boldsymbol{z}]=\boldsymbol{z}$ is a proper algebraic system if $\alpha$ is a proper algebraic polynomial.
} 
Lemma 3.15. If $p: M(\mathbb{C})^{g} \rightarrow M(\mathbb{C})^{g}$ is a bijective free polynomial and $q$ is the inverse of $p$ then for each $n$, there exists a free polynomial $r_{n}$ such that $q(X)=r_{n}(X)$ for all $X \in M_{n}(\mathbb{C})^{g}$.

Proof. This is part of Theorem A(iv) and a proof can be found in [Pas14], however, for the reader's convenience we present a more detailed argument showing $q$ agrees with a free polynomial on each $M_{n}(\mathbb{C})^{g}$.

Let $\pi: M_{n}(\mathbb{C})^{g} \rightarrow \mathbb{C}^{g n^{2}}$ be the canonical isomorphism. Since $p$ is bijective, $p[n]: M_{n}(\mathbb{C})^{g} \rightarrow M_{n}(\mathbb{C})^{g}$ is bijective and we may view $p[n]$ as a polynomial in $g n^{2}$ variables. That is, $\pi \circ p \circ \pi^{-1}$ is a bijective (commutative) polynomial, hence by the classical Grothendieck theorem, $\pi \circ p \circ \pi^{-1}$ has a (commutative) polynomial inverse $\hat{q}: \mathbb{C}^{g n^{2}} \rightarrow \mathbb{C}^{g n^{2}}$.

Since $p$ is a bijective free polynomial, $q$ is free analytic by Theorem 3.1 in [HKM11], hence $q[n]: M_{n}(\mathbb{C})^{g} \rightarrow M_{n}(\mathbb{C})^{g}$ is analytic and there is a power series, $R=\sum_{m=0}^{\infty} \sum_{|w|=m} r_{w} w$ such that $R$ converges on $M_{n}(\mathbb{C})^{g}$ and $R(X)=q[n](X)$ for all $X \in M_{n}(\mathbb{C})^{g}$. In particular, $\left(\pi^{-1} \circ \hat{q} \circ \pi\right)(X)=R(X)=q[n](X)$ for all $X \in M_{n}(\mathbb{C})^{g}$, hence $\operatorname{deg}(\hat{q})=\operatorname{deg}(q[n])$. Set $\hat{R}=\sum_{m=0}^{\operatorname{deg}(\hat{q})} \sum_{|w|=m} r_{w} w$ and note $\hat{R}(X)=q[n](X)$ for all $X \in M_{n}(\mathbb{C})^{g}$. Since $\hat{R}$ is a free polynomial, we conclude $q$ agrees with a free polynomial on $M_{n}(\mathbb{C})^{g}$.

Remark 3.16. If $p$ is a bijective free polynomial with a free polynomial inverse $q$, then both $J_{p}$ and $J_{q}$ are polynomial matrices and $J_{q}(p(\boldsymbol{x}))$ also is a polynomial matrix. Observe $I_{g}=J_{q \circ p}(\boldsymbol{x})=J_{p}(\boldsymbol{x}) J_{q}(p(\boldsymbol{x}))$, thus $J_{p}^{-1} \in M_{g}(\mathbb{C}\langle\mathbb{x}\rangle)$ since $J_{p}(\boldsymbol{x})^{-1}=J_{q}(p(\boldsymbol{x}))$.

Remark 3.16 is an expected consequence of the Jacobian matrix satisfying the chain rule and Corollary 1.4 in [Reu92] offers a slightly different proof. Certainly if $p$ is invertible then it is bijective, however Example 3.19 shows that $J_{p}, J_{p}^{-1} \in$ $M_{g}(\mathbb{C}\langle\mathbb{x}\rangle)$ is not sufficient for $p^{-1}$ to be a polynomial. In that sense there is no Jacobian conjecture for the noncommutative Jacobian matrix.

On the other hand, Theorem 3.17 profits from a noncommutative Nullstellensatz in [HM04] to prove the Jacobian matrix of an injective free polynomial is invertible over $M_{g}(\mathbb{C}\langle\mathbb{x}\rangle)$. Before proving the theorem, we first state the noncommutative Nullstellensatz (proved by George Bergman), Theorem 6.3 in [HM04].

Theorem B. Let $\mathcal{P} \subset \mathbb{C}\langle\mathbb{x}\rangle$ be finite and let $s \in \mathbb{C}\langle\mathbb{x}\rangle$. Let d denote the maximum of the $\operatorname{deg}(s)$ and $\{\operatorname{deg}(p): p \in \mathcal{P}\}$. There exists a complex Hilbert space $\mathcal{H}$ of dimension $\sum_{j=0}^{d} g^{j}$, such that, if

$$
\begin{gathered}
s(X) v=0 \\
\text { whenever } X=\left(X_{1}, \ldots, X_{g}\right) \in \mathcal{B}(\mathcal{H})^{g}, v \in \mathcal{H} \text {, and } \\
p(X) v=0 \text { for all } p \in \mathcal{P},
\end{gathered}
$$

then $s$ is in the left ideal generated by $\mathcal{P}$.

Theorem 3.17. If $p$ is a bijective polynomial mapping, then $J_{p}^{-1}$ is a polynomial (matrix).

Proof. Let $d=\max \left\{\operatorname{deg}\left(p^{j}\right)\right\}$ and set $N=g^{d+1}$. Lemma 3.15 tells us $q[N]$ agrees with a free polynomial. Suppose $s=\left(s^{1}, \ldots, s^{g}\right)$ is a free polynomial such that 
$q(X)=s(X)$ for all $X \in M_{N}(\mathbb{C})^{g}$. In particular, each $s^{j}$ has no constant term. For each $1 \leq j \leq g$ write

$$
s^{j}=\sum_{m=1}^{\operatorname{deg}(s)} \sum_{|w|=m} \sigma_{w}^{j} w
$$

and observe

$$
X_{j}=s^{j}(p(X))=\sum_{m=1}^{\operatorname{deg}(s)} \sum_{|w|=m} \sigma_{w}^{j} w(p(X)) .
$$

Take $X=\left(X_{1}, \ldots, X_{g}\right) \in M_{N}(\mathbb{C})^{g}$ and $v \in \mathbb{C}^{N}$ such that $v^{T} p^{j}(X)=0$ for all $1 \leq j \leq g$. Hence $v^{T} w(p(X))=0$ for all $|w|>0$ since $v^{T} p^{j}(X)=0$ for each $1 \leq j \leq g$. Thus,

$$
v^{T} X_{j}=v^{T} s^{j}(p(X))=\sum_{m=1}^{\operatorname{deg}(s)} \sum_{|w|=m} \sigma_{w}^{j} v^{T} w(p(X))=0 .
$$

By Theorem $\mathrm{B}, x_{j}$ is contained in the right ideal generated by $p^{1}, \ldots, p^{g}$, that is, there exist polynomials $\mu_{i, j}$ such that $x_{j}=\sum_{i=1}^{g} p^{i}(\boldsymbol{x}) \mu_{i, j}(\boldsymbol{x})$. Let $R=\left(\mu_{i, j}\right)_{i, j=1}^{g} \in$ $M_{g}(\mathbb{C}\langle\mathbb{x}\rangle)$ and observe

$$
\boldsymbol{x}=p(\boldsymbol{x}) R(\boldsymbol{x})=\boldsymbol{x} J_{p}(\boldsymbol{x}) R(\boldsymbol{x}) .
$$

Therefore $J_{p}^{-1}=R \in M_{g}(\mathbb{C}\langle\mathbb{x}\rangle)$.

Example 3.18. Let

$$
N=\left(\begin{array}{ll}
-1 & 1 \\
-1 & 1
\end{array}\right)
$$

and set

$$
\begin{aligned}
& p(\boldsymbol{x})=\boldsymbol{x}\left(I_{2}-N x_{1}\right) \\
& =\left(\begin{array}{ll}
x_{1}, & x_{2}
\end{array}\right)\left(\begin{array}{cc}
1+x_{1} & -x_{1} \\
x_{1} & 1-x_{1}
\end{array}\right) \\
& =\left(x_{1}+x_{1}^{2}+x_{2} x_{1}, \quad x_{2}-x_{1}^{2}-x_{2} x_{1}\right) \text {. }
\end{aligned}
$$

Observe, $J_{p}=I_{2}-N x_{1}$, and that $N^{2}=0$. Hence, $J_{p}^{-1}=I_{2}+N x_{1}$ and

$$
\begin{aligned}
& \mathrm{qI}(\boldsymbol{x})[\boldsymbol{z}]=\boldsymbol{x}\left(I_{2}+N z_{1}\right) \\
& =\left(x_{1}-x_{1} z_{1}-x_{2} z_{1}, \quad x_{2}+x_{1} z_{1}+x_{2} z_{1}\right) \\
& =\left(x_{1}-\left(x_{1}+x_{2}\right) z_{1}, \quad x_{2}+\left(x_{1}+x_{2}\right) z_{1}\right) .
\end{aligned}
$$

However, $p$ is not even injective on $\mathbb{C}^{2}$ since $p(-1 / 2,-1 / 2)=(0,1)=p(0,1)$.

Note,

$$
\left(\begin{array}{ll}
0 & 1 \\
0 & 0
\end{array}\right)=\left(\begin{array}{cc}
0 & 1 \\
-1 & 1
\end{array}\right)\left(\begin{array}{cc}
-1 & 1 \\
-1 & 1
\end{array}\right)\left(\begin{array}{cc}
1 & -1 \\
1 & 0
\end{array}\right)
$$

that is, $N$ is similar to a strictly upper triangular nilpotent matrix. Thus, conjugation of a Jacobian matrix by a similarity does not preserve the desirable properties of the Jacobian matrix. 
In some sense, the noncommutative Jacobian matrix attempts to linearize polynomial mappings so that a reasonable structure is preserved via composition. In fact, if $p$ is a formal power series mapping, then $J_{p}$ is invertible if and only if $p$ is locally invertible at 0 , a statement reminiscent of the inverse function theorem. Hence, $J_{p}^{-1}$ is a linear approximation of $p^{-1}$ at 0 , explaining why we can iteratively construct $p^{-1}$ from $J_{p}^{-1}$. However, Example 3.19 shows how the Jacobian matrix can fail to witness the non-injectivity of a polynomial.

In subsection 6.3 we construct the hypo-Jacobian matrix of a free polynomial, a matrix whose invertibility exactly captures the injectivity or non-injectivity of the free polynomial.

Example 3.19. This example is investigated in [Reu92] and it shows that there is no Jacobian conjecture with the noncommutative Jacobian matrix. Let $p(\boldsymbol{x})=$ $\left(x_{1}, x_{2}-x_{1} x_{2} x_{1}\right)$, and observe

$$
J_{p}(\boldsymbol{x})=\left(\begin{array}{cc}
1 & -x_{2} x_{1} \\
0 & 1
\end{array}\right), \quad J_{p}^{-1}(\boldsymbol{x})=\left(\begin{array}{cc}
1 & x_{2} x_{1} \\
0 & 1
\end{array}\right) .
$$

Note $p$ is not bijective and $\mathbb{q}(\boldsymbol{x})[\boldsymbol{z}]=\left(x_{1}, x_{2}+x_{1} z_{2} z_{1}\right)$. It is straightforward to verify that $\mathbb{q}^{\circ k}(\boldsymbol{x})[\boldsymbol{z}]=\left(x_{1}, x_{2}+x_{1}^{k} z_{2} z_{1} x_{1}^{k-1}+\sum_{j=1}^{k-1} x_{1}^{j} x_{2} x_{1}^{j}\right)$ and $q(\boldsymbol{x})=$ $\left(x_{1}, \sum_{j=0}^{\infty} x_{1}^{j} x_{2} x_{1}^{j}\right)$. Thus $q$ is certainly not a polynomial.

Recall $\mathbb{q}^{\circ k}(\boldsymbol{x})[\boldsymbol{z}]=\mathfrak{q}^{k}(\boldsymbol{x})+r^{n}(\boldsymbol{x})[\boldsymbol{z}]$. For this example, $\mathfrak{q}^{k}(\boldsymbol{x})=\left(x_{1}, \sum_{j=0}^{k-1} x_{1}^{j} x_{2} x_{1}^{j}\right)$ while $r^{k}(\boldsymbol{x})[\boldsymbol{z}]=\left(0, x_{1}^{k} z_{2} x_{1}^{k}\right)$ and $\mathbb{d}_{q}^{k}=2 k+1$. In particular, $\operatorname{deg}\left(\mathfrak{q}^{k}\right)=2 k-1$ is strictly increasing with $k$, immediately discounting $q$ from being a polynomial, however $q$ is an algebraic function.

In fact, if $p$ is any free polynomial whose auxiliary inverse, $\mathbb{q}$, is a polynomial then the only way for the $p$ to have a non-polynomial inverse is if the situation above occurs, that is, $\operatorname{deg}\left(\mathfrak{q}^{k}\right)$ is a strictly increasing sequence. Section 4 deals with exactly this.

\section{Free Derivatives AND the Linearization of THE AUXiLiary inverse}

In this section we establish conditions that guarantee $q$, the compositional inverse of $p$, is a polynomial. We use Theorem A to linearize q, the auxiliary inverse of $p$, in terms of $z_{1}, \ldots, z_{g}$. This linearization has the caveat that we introduce $g$-'dummy' variables.

4.1. Polynomial criteria. We begin by recalling a few facts about auxiliary inverses. By Theorem 3.17 we know if $p$ is a bijective free polynomial with $p(0)=0$ then $J_{p}$ and $J_{p}^{-1}$ are matrices of free polynomials. Recall from Definition 3.10 that $\mathrm{q}^{k}$, the $k^{\text {th }}$ auxiliary inverse of $p$, is given by $\mathbb{q}^{\circ k}(\boldsymbol{x})[\boldsymbol{z}]=\mathfrak{q}^{k}(\boldsymbol{x})+r^{k}(\boldsymbol{x})[\boldsymbol{z}]$, where $\operatorname{deg}\left(\mathfrak{q}^{k}\right)<\mathbb{d}_{q}^{k}$. Furthermore, by Lemma 3.9 we know $\mathbb{d}_{q}^{k} \nearrow \infty$. Proposition 3.14 tells us that $q$, the inverse of $p$, is the unique solution of $\mathbb{q}(\boldsymbol{x})[q(\boldsymbol{x})]=q(\boldsymbol{x})$. Since $q=\lim _{k \rightarrow \infty} \mathfrak{q}^{k}$, if $q$ were actually a free polynomial then we would expect a large degree gap to appear in the monomials of $\mathrm{qI}^{\circ k}$. This is precisely what Lemma 4.1 deals with.

Lemma 4.1. Suppose $b \in\left(\mathbb{C}\langle\mathbb{x}\rangle_{+}\right)^{g}$ and $a \in\left(\mathbb{C}\langle\mathbb{x}\rangle_{+}\right)^{g}$ are compositional inverses, $\mathbf{a} \in\left(\mathbb{C}\langle\mathbb{x} \cup \mathbb{Z}\rangle_{+}\right)^{g}$ is a proper algebraic polynomial, and $\mathbf{a}(\boldsymbol{x})[a(\boldsymbol{x})]=a(\boldsymbol{x})$. Let $\mathrm{a}^{\circ k}(\boldsymbol{x})[\boldsymbol{z}]=\mathfrak{a}^{k}(\boldsymbol{x})+\alpha^{k}(\boldsymbol{x})[\boldsymbol{z}]$ as in (3.3) and $\mathbb{d}_{a}^{k}=\mathbb{d}_{z}\left(\mathrm{a}^{\circ k}\right)$ as in (3.1). The following are equivalent; 
(i) a is a polynomial;

(ii) $a=\mathfrak{a}^{m}$ for some $m \in \mathbb{Z}^{+}$;

(iii) $\mathbb{d}_{a}^{N}>\operatorname{deg}\left(\mathfrak{a}^{N}\right) \operatorname{deg}(b)$ for some $N \in \mathbb{Z}^{+}$.

Proof. Recall from Lemma 3.9 that $\lim _{k \rightarrow \infty} \mathfrak{a}^{k}=a, \mathbf{a}(\boldsymbol{x})[a(\boldsymbol{x})]=a(\boldsymbol{x})$ and $\mathbb{d}_{a}^{k}$ is either always strictly increasing or is strictly increasing until it becomes constant at infinity. We note $\mathbf{a}^{\circ k+1}(\boldsymbol{x})[a(\boldsymbol{x})]=\mathbf{a}^{\circ k}(\boldsymbol{x})[\mathbf{a}(\boldsymbol{x})[a(\boldsymbol{x})]]=\mathbf{a}^{\circ k}(\boldsymbol{x})[a(\boldsymbol{x})]$, implying

$$
\mathrm{a}^{\circ k}(\boldsymbol{x})[a(\boldsymbol{x})]=a(\boldsymbol{x})
$$

for all $k \geq 1$. Next, composing with $b(\boldsymbol{x})$ yields $\mathbf{a}^{\circ k}(b(\boldsymbol{x}))[a(b(\boldsymbol{x}))]=a(b(\boldsymbol{x}))=\boldsymbol{x}$.

$(i) \Rightarrow($ ii $)$. Suppose $a$ is a polynomial. By Item (ii) in Lemma 3.9, if $k \geq \operatorname{deg}(a)$, then $\mathbb{d}_{a}^{k}>\operatorname{deg}(a)$. Hence

$$
a(\boldsymbol{x})=\mathrm{a}^{\circ k}(\boldsymbol{x})[a(\boldsymbol{x})]=\mathfrak{a}^{k}(\boldsymbol{x})+\alpha^{k}(\boldsymbol{x})[a(\boldsymbol{x})] .
$$

However, by the definition of $\alpha^{k}$, the minimum possible length of any word appearing in $\alpha^{k}$ is $\mathbb{d}_{a}^{k}>\operatorname{deg}(a)$. Thus $\alpha^{k}(\boldsymbol{x})[a(\boldsymbol{x})]=0$ and $\mathfrak{a}^{k}=a$.

(ii) $\Rightarrow\left(\right.$ iii). Suppose $a=\mathfrak{a}^{m}$ for some $m \in \mathbb{Z}^{+}$. If $n \geq m$ then items (iii) and (iv) in Lemma 3.9 imply $\mathfrak{a}^{n}-\mathfrak{a}^{m}$ contains no monomials of length less than or equal to $\operatorname{deg}\left(\mathfrak{a}^{m}\right)$. However, $\mathfrak{a}^{m}=a$, hence we must have $\mathfrak{a}^{m}=\mathfrak{a}^{n}=a$, for all $n \geq m$. Thus, the sequence $\operatorname{deg}\left(\mathfrak{a}^{n}\right) \operatorname{deg}(b)$ is constant for $n \geq m$. On the other hand, $\mathbb{d}_{a}^{n}$ is either always strictly increasing or is strictly increasing until it becomes constant at infinity. Therefore, there is some $N$ such that $\mathbb{d}_{a}^{N}>\operatorname{deg}(a) \operatorname{deg}(b)=\operatorname{deg}\left(\mathfrak{a}^{N}\right) \operatorname{deg}(b)$.

(iii) $\Rightarrow(\mathrm{i})$. Suppose there is some $N$ such that $\mathbb{d}_{a}^{N}>\operatorname{deg}\left(\mathfrak{a}^{N}\right) \operatorname{deg}(b)$. Substituting $b(\boldsymbol{x})$ for $\boldsymbol{x}$ in (4.1),

$$
\boldsymbol{x}=\mathrm{a}^{\circ N}(b(\boldsymbol{x}))[\boldsymbol{x}]=\mathfrak{a}^{N}(b(\boldsymbol{x}))+\alpha^{N}(b(\boldsymbol{x}))[\boldsymbol{x}] .
$$

However,

$$
\operatorname{deg}\left(\mathfrak{a}^{N}(b(\boldsymbol{x}))\right) \leq \operatorname{deg}\left(\mathfrak{a}^{N}\right) \operatorname{deg}(b)<\mathbb{d}_{q}^{N},
$$

and the minimum degree of any monomial appearing in $\alpha^{N}(b(\boldsymbol{x}))[\boldsymbol{x}]$ is greater than $\mathbb{d}_{q}^{N}$, implying $\alpha^{N}(b(\boldsymbol{x}))[\boldsymbol{x}]=0$. Thus $\mathfrak{a}^{N}(b(\boldsymbol{x}))=\boldsymbol{x}$, therefore $a=\mathfrak{a}^{N}$ is a polynomial.

Remark 4.2. Suppose $p \in\left(\mathbb{C}\langle\mathbb{x}\rangle_{+}\right)^{g}$ with $J_{p}, J_{p}^{-1} \in M_{g}(\mathbb{C}\langle\mathbb{x}\rangle)$. Let q be the auxiliary inverse of $p$ and let $q$ be the compositional inverse of $p$. Recall that since $q$ is a polynomial and $\mathbb{d}_{z}(\mathfrak{\mathbb { }})>1, \mathfrak{q}(\boldsymbol{x})[\boldsymbol{z}]$ is a proper algebraic polynomial and $q$ is the unique algebraic function such that $\mathbb{q}(\boldsymbol{x})[q(\boldsymbol{x})]=q(\boldsymbol{x})$. Thus Lemma 4.1 applies to qI and $q$.

It should be noted that if $a=\mathfrak{a}^{N}$ for some $N$ then we cannot conclude $\alpha^{N}=0$. Example 4.3 describes a bijective free polynomial $p$ with a free polynomial inverse $q$, such that $\mathbb{q}^{\circ k}(\boldsymbol{x})[\boldsymbol{z}] \neq q(\boldsymbol{x})$, i.e. $r^{k}(\boldsymbol{x})[\boldsymbol{z}] \neq 0$ for any $k \geq 1$.

Example 4.3. Let $p^{1}, p^{2} \in \mathbb{C}\left\langle x_{1}, x_{2}\right\rangle$ with $p^{1}=\left(x_{1}, x_{2}+x_{1}^{2}\right)$, and $p^{2}=\left(x_{1}+x_{2}^{2}, x_{2}\right)$. Both are bijective with $q^{1}=\left(x_{1}, x_{2}-x_{1}^{2}\right)$ and $q^{2}=\left(x_{1}-x_{2}^{2}, x_{2}\right)$ as their respective inverses. Their composition $p=p^{1} \circ p^{2}=\left(x_{1}+x_{2}^{2}, x_{2}+\left(x_{1}+x_{2}^{2}\right)^{2}\right)$ has inverse $q=q^{2} \circ q^{1}=\left(x_{1}-\left(x_{2}-x_{1}^{2}\right)^{2}, x_{2}-x_{1}^{2}\right)$.

Since

$$
p^{1}=\left(\begin{array}{ll}
x_{1} & x_{2}
\end{array}\right)\left(\begin{array}{cc}
1 & -x_{1} \\
0 & 1
\end{array}\right)^{-1} \quad \text { and } \quad p^{2}=\left(\begin{array}{ll}
x_{1} & x_{2}
\end{array}\right)\left(\begin{array}{cc}
1 & 0 \\
-x_{2} & 1
\end{array}\right)^{-1}
$$

we have,

$$
p=\left(\begin{array}{ll}
x_{1} & x_{2}
\end{array}\right)\left(\begin{array}{cc}
1 & 0 \\
-x_{2} & 1
\end{array}\right)^{-1}\left(\begin{array}{cc}
1 & -\left(p^{2}\right)_{1} \\
0 & 1
\end{array}\right)^{-1}=\left(\begin{array}{ll}
x_{1} & x_{2}
\end{array}\right)\left(\begin{array}{cc}
1 & x_{1}+x_{2}^{2} \\
x_{2} & 1+x_{2}\left(x_{1}+x_{2}^{2}\right)
\end{array}\right),
$$


and

$$
\begin{aligned}
\mathrm{T}(\boldsymbol{x})[\boldsymbol{z}] & =\left(\begin{array}{ll}
x_{1} & x_{2}
\end{array}\right)\left(\begin{array}{cc}
1 & -\left(z_{1}+z_{2}^{2}\right) \\
0 & 1
\end{array}\right)\left(\begin{array}{cc}
1 & 0 \\
-z_{2} & 1
\end{array}\right) \\
& =\left(x_{1}+x_{1}\left(z_{1}+z_{2}^{2}\right) z_{2}-x_{2} z_{2}, x_{2}-x_{1}\left(z_{1}+z_{2}^{2}\right)\right) .
\end{aligned}
$$

In this case a gap between $\mathfrak{q}^{k}$ and $r^{k}(\boldsymbol{x})[\boldsymbol{z}]$ forms rather quickly and the true inverse is extracted quite easily. However each iterate of $\mathbb{q}^{k}$ will have a $z$-term.

4.2. Free derivatives and scions. We now introduce the formal directional derivative as was done in [Pas14] and similarly in [HKM12] and [HKM11].

Definition 4.4. Let $\mathrm{y}=\left\{y_{1}, \ldots, y_{g}\right\}$ be a set of noncommuting indeterminates distinct from $\mathbb{x}$ and let $\boldsymbol{y}=\left(y_{1}, \ldots, y_{g}\right)$ be considered as a row vector. We define the free derivative $D: \mathbb{C}\langle\langle\mathbb{x}\rangle\rangle \rightarrow \mathbb{C}\langle\langle\mathbb{x} \cup \mathbb{y}\rangle\rangle$ by its action on monomials and then extend it linearly and continuously. Define

$$
D x_{i}(\boldsymbol{x})[\boldsymbol{y}]=y_{i}
$$

and require

(i) $D(k \eta+\mu)(\boldsymbol{x})[\boldsymbol{y}]=k D \eta(\boldsymbol{x})[\boldsymbol{y}]+D \mu(\boldsymbol{x})[\boldsymbol{y}]$;

(ii) $D(\eta \mu)(\boldsymbol{x})[\boldsymbol{y}]=D \eta(\boldsymbol{x})[\boldsymbol{y}] \mu(\boldsymbol{x})+\eta(\boldsymbol{x}) D \mu(\boldsymbol{x})[\boldsymbol{y}]$,

for all formal power series $\eta, \mu \in \mathbb{C}\langle\langle\mathbb{x}\rangle\rangle$. Consequently, for all $\nu \in\left(\mathbb{C}\left\langle\langle\mathbb{x}\rangle_{+}\right)^{g}\right.$ we have

$$
D(\eta \circ \nu)(\boldsymbol{x})[\boldsymbol{y}]=D \eta(\nu(\boldsymbol{x}))\left[\left(D \nu_{1}(\boldsymbol{x})[\boldsymbol{y}], \ldots, D \nu_{g}(\boldsymbol{x})[\boldsymbol{y}]\right)\right] .
$$

Observe $D \eta(\boldsymbol{x})[\boldsymbol{y}]$ is linear in $\boldsymbol{y}$, that is, $D \eta(\boldsymbol{x})[k \boldsymbol{y}+\boldsymbol{z}]=k D \eta(\boldsymbol{x})[\boldsymbol{y}]+D \eta(\boldsymbol{x})[\boldsymbol{z}]$. The linearity of the free derivative allows us to define $D$ on matrices of formal power series. If $A \in M_{m \times n}(\mathbb{C}\langle\langle\mathbb{x}\rangle\rangle)$ then define $D: M_{m \times n}\left(\mathbb{C}\langle\langle\mathbb{x}\rangle) \rightarrow M_{m \times n}(\mathbb{C}\langle\langle\mathbb{x} \cup \mathbb{y}\rangle)\right.$ by

$$
D A(\boldsymbol{x})[\boldsymbol{y}]=\left(D A_{i, j}(\boldsymbol{x})[\boldsymbol{y}]\right)_{i, j=1}^{m, n} .
$$

In particular $D$ extends to row vectors in the obvious way.

Remark 4.5. The derivative in free analysis is defined below, and is almost a pure matrix result. Suppose $\mathcal{U}$ is a free domain (hence open) and $\eta: \mathcal{U} \rightarrow M(\mathbb{C})^{g}$ is an analytic free map. For any small enough $H \in M(\mathbb{C})^{g}$,

$$
\eta\left(\begin{array}{cc}
X & H \\
0 & X
\end{array}\right)=\left(\begin{array}{cc}
\eta(X) & D \eta(X)[H] \\
0 & \eta(X)
\end{array}\right) .
$$

It turns out, there is a strong connection between the free derivative in (4.2) and the formal power series derivative in Definition 4.4 (justifying the redundant use of $D)$.

If $\lambda: M(\mathbb{C})^{g} \rightarrow M(\mathbb{C})^{g}$ is a free analytic mapping, $\Lambda \in(\mathbb{C}\langle(\mathbb{x}\rangle\rangle)^{g}$ is a formal power series that converges on $M(\mathbb{C})^{g}$, and $\Lambda(X)=\lambda(X)$ for all $X \in M(\mathbb{C})^{g}$, then $D \Lambda \in\left(\mathbb{C}\langle\langle\mathbb{x} \cup \mathbb{y}\rangle)^{g}\right.$ converges on $M(\mathbb{C})^{2 g}$ and $D \lambda(X)[Y]=D \Lambda(X)[Y]$ for all $X, Y \in M(\mathbb{C})^{g}$.

To see this, let $\Lambda=\sum_{m=0}^{\infty} \sum_{|w|=m} L_{w} w$ and $\Lambda_{N}=\sum_{m=0}^{N} \sum_{|w|=m} L_{w} w$. Since $\Lambda_{N} \in(\mathbb{C}\langle\mathbb{x}\rangle)^{g}, D \Lambda_{N} \in(\mathbb{C}\langle\mathbb{x} \cup \mathbb{y}\rangle)^{g}$, thus $D \Lambda_{N}(X)[Y]$ exists for all $(X, Y) \in M(\mathbb{C})^{2 g}$. Let $Z=\left(\begin{array}{cc}X & Y \\ 0 & X\end{array}\right)$. By Proposition 6 in [Pas14],

$$
\Lambda_{N}(Z)=\Lambda_{N}\left(\begin{array}{cc}
X & Y \\
0 & X
\end{array}\right)=\left(\begin{array}{cc}
\Lambda_{N}(X) & D \Lambda_{N}(X)[Y] \\
0 & \Lambda_{N}(X)
\end{array}\right) .
$$


The sequence of polynomials $\left(D \Lambda_{N}\right)$ converges to $D \Lambda$ in the metric topology on $\left(\mathbb{C}\langle\langle\mathbb{x} \cup \mathbb{y}\rangle)^{g}\right.$, thus $D \Lambda_{N}(X)[Y]$ converges to $D \Lambda(X)[Y]$, since $\Lambda_{N}(Z)$ converges to $\Lambda(Z)$. Hence,

$$
\Lambda(Z)=\Lambda\left(\begin{array}{cc}
X & Y \\
0 & X
\end{array}\right)=\left(\begin{array}{cc}
\Lambda(X) & D \Lambda(X)[Y] \\
0 & \Lambda(X)
\end{array}\right)
$$

and $D \Lambda(X)[Y]=D \lambda(X)[Y]$.

Thus, to find the derivative of a free analytic function with a formal power series, it is sufficient to find the derivative of the formal power series and then evaluate where desired.

Example 4.6. We present a few formal power series and their corresponding derivatives. If $p\left(x_{1}, x_{2}\right)=x_{1} x_{2}-x_{2} x_{1}$ then $D p\left(x_{1}, x_{2}\right)\left[y_{1}, y_{2}\right]=y_{1} x_{2}+x_{1} y_{2}-$ $y_{2} x_{1}-x_{2} y_{1}$. Next, if $r\left(x_{1}\right)=\left(1-x_{1}\right)^{-1}$ then $\operatorname{Dr}\left(x_{1}\right)\left[y_{1}\right]=\left(1-x_{1}\right)^{-1} y_{1}\left(1-x_{1}\right)^{-1}$. Finally, if $s^{1}\left(x_{1}, x_{2}\right)=\left(x_{1}, x_{2}+x_{1}^{2}\right)$ and $s^{2}\left(x_{1}, x_{2}\right)=\left(x_{2}, x_{1}+x_{2}\right)$ then

$$
D s^{1}\left(x_{1}, x_{2}\right)\left[y_{1}, y_{2}\right]=\left(y_{1}, y_{2}+x_{1} y_{1}+y_{1} x_{1}\right), \quad D s^{2}\left(x_{1}, x_{2}\right)\left[y_{1}, y_{2}\right]=\left(y_{2}, y_{1}+y_{2}\right)
$$

and

$$
\begin{aligned}
D\left(s^{1} \circ s^{2}\right)\left(x_{1}, x_{2}\right)\left[y_{1}, y_{2}\right] & =D s^{1}\left(s^{2}\left(x_{1}, x_{2}\right)\right)\left[D s^{2}\left(x_{1}, x_{2}\right)\left[y_{1}, y_{2}\right]\right] \\
& =\left(y_{2},\left(y_{1}+y_{2}\right)+x_{2} y_{2}+y_{2} x_{2}\right) .
\end{aligned}
$$

Before proceeding with our investigation of the free derivative, we stop to quickly prove the implicit function theorem for nc formal power series. For an analytic approach to the implicit function theorem for $M(\mathbb{C})^{g}$ see [AM16].

Definition 4.7. Suppose $\mathbb{Z}=\left\{z_{1}, \ldots, z_{h}\right\}$ and $f(\boldsymbol{x}, \boldsymbol{z}) \in\left(\mathbb{C}\langle\langle\mathbb{\mathbb { x }} \cup \mathbb{Z}\rangle)^{h}\right.$. Define

$$
\frac{\partial f}{\partial \boldsymbol{z}}=\left(D f_{i}(\boldsymbol{x}, \boldsymbol{z})\left[0, e_{j}\right]\right)_{i, j=1}^{h} \in M_{h}(\mathbb{C}\langle\langle\mathbb{x} \cup \mathbb{Z}\rangle),
$$

where $e_{j}$ is the standard vector with a 1 in the $i^{\text {th }}$ position and 0 elsewhere.

Theorem 4.8 ((Implicit function theorem)). Suppose $f(\boldsymbol{x}, \boldsymbol{z}) \in\left(\mathbb{C}\langle\langle\mathbb{\mathbb { x }} \cup \mathbb{Z}\rangle)^{h}\right.$. If $f(0,0)=0$ and $\partial f / \partial \boldsymbol{z}(0,0) \in M_{h}(\mathbb{C})$ is invertible, then there exists a unique $\mathfrak{g} \in(\mathbb{C}\langle(\mathbb{x}\rangle))^{h}$ such that $\mathfrak{g}(0)=0$ and $f(\boldsymbol{x}, \mathfrak{g}(\boldsymbol{x}))=0$.

Proof. Since $f(0,0)=0$, we see that $f(\boldsymbol{x}, \boldsymbol{z})$ has no constant terms. By composing with an appropriate change of variables, we may assume $\partial f / \partial \boldsymbol{z}(0,0)=I_{h}$. Hence, the coefficient of each $z_{i}$ term in $f_{j}$ is $\delta_{i, j}$, the Kronecker delta. Set $\hat{f}(\boldsymbol{x})[\boldsymbol{z}]=$ $\boldsymbol{z}-f(\boldsymbol{x}, \boldsymbol{z})$ and note $\hat{f}$ satisfies the conditions of Lemma 3.9. Thus, there exists a unique $\mathfrak{g} \in(\mathbb{C}\langle\langle\mathbb{x}\rangle\rangle)^{h}$ such that $\mathfrak{g}(0)=0$ and $\hat{f}(\boldsymbol{x})[\mathfrak{g}(\boldsymbol{x})]=\mathfrak{g}(\boldsymbol{x})$. Finally, since $f(\boldsymbol{x}, \boldsymbol{z})=\boldsymbol{z}-\hat{f}(\boldsymbol{x})[\boldsymbol{z}]$,

$$
f(\boldsymbol{x}, \mathfrak{g}(\boldsymbol{x}))=\mathfrak{g}(\boldsymbol{x})-\hat{f}(\boldsymbol{x})[\mathfrak{g}(\boldsymbol{x})]=\mathfrak{g}(\boldsymbol{x})-\mathfrak{g}(\boldsymbol{x})=0,
$$

and the uniqueness of $\mathfrak{g}$ for $\hat{f}$ implies $\mathfrak{g}$ is the unique formal power series satisfying both $\mathfrak{g}(0)=0$ and $(4.3)$.

Definition 4.9. Suppose $p \in(\mathbb{C}\langle\mathbb{x}\rangle)^{g}$. We define the scion of $p, F \in(\mathbb{C}\langle\mathbb{x} \cup \mathbb{y}\rangle)^{2 g}$, by $F(\boldsymbol{x}, \boldsymbol{y})=(D p(\boldsymbol{y})[\boldsymbol{x}], \boldsymbol{y})$. Furthermore, if we view $p$ as a free polynomial from $M(\mathbb{C})^{g}$ to $M(\mathbb{C})^{g}$, then $F: M(\mathbb{C})^{2 g} \rightarrow M(\mathbb{C})^{2 g}$ is a free polynomial and $F(X, Y)=(D p(Y)[X], Y)$.

Of particular importance is the fact that $F$ is $\boldsymbol{x}$-linear, that is, $F(\boldsymbol{x}+\boldsymbol{z}, \boldsymbol{y})=$ $F(\boldsymbol{x}, \boldsymbol{y})+F(\boldsymbol{z}, \boldsymbol{y})$. Moreover, $D F(\boldsymbol{x}, \boldsymbol{y})[\boldsymbol{z}, \boldsymbol{w}]$ is automatically $\boldsymbol{z}$-linear. 
Proposition 4.10. Suppose $p$ is a free polynomial. If $F$ is the scion of $p$, then $p$ is bijective if and only if $F$ is bijective.

Proof. Suppose $J_{p}$ is the Jacobian matrix of $p$. Since $p(\boldsymbol{x})=\boldsymbol{x} J_{p}(\boldsymbol{x})$, the Jacobian matrix of $F$ is given by

$$
J_{F}(\boldsymbol{x}, \boldsymbol{y})=\left(\begin{array}{cc}
J_{p}(\boldsymbol{y}) & 0 \\
D J_{p}(\boldsymbol{y})[\boldsymbol{x}] & I
\end{array}\right)
$$

We note that $F(\boldsymbol{x}, \boldsymbol{y})=(\boldsymbol{x}, \boldsymbol{y}) J_{F}(\boldsymbol{x}, \boldsymbol{y})$. Let $\boldsymbol{h}=\left(h_{1}, \ldots, h_{g}\right)$ and $\boldsymbol{k}=\left(k_{1}, \ldots, k_{g}\right)$ be $g$-tuples of noncommuting indeterminates treated as row vectors. Consider

$$
\begin{aligned}
D F & (\boldsymbol{x}, \boldsymbol{y})[\boldsymbol{h}, 0] \\
& =\left(\boldsymbol{h} J_{p}(\boldsymbol{y})+\boldsymbol{x} D J_{p}(\boldsymbol{y})[0]+0 D J_{p}(\boldsymbol{y})[\boldsymbol{x}]+\boldsymbol{y} D\left(D J_{p}(\boldsymbol{y})[\boldsymbol{x}]\right)[\boldsymbol{h}, 0], 0\right) \\
& =\left(\boldsymbol{h} J_{p}(\boldsymbol{y})+0+0+\boldsymbol{y} D J_{p}(\boldsymbol{y})[\boldsymbol{h}], 0\right) \\
& =(D p(\boldsymbol{y})[\boldsymbol{h}], 0) .
\end{aligned}
$$

The motivation for why $D\left(D J_{p}(\boldsymbol{y})[\boldsymbol{x}]\right)[\boldsymbol{h}, 0]=D J_{p}(\boldsymbol{y})[\boldsymbol{h}]$ hinges on the $\boldsymbol{x}$-linearity of $D J_{p}(\boldsymbol{y})[\boldsymbol{x}]$. To clarify this point we demonstrate on a monomial $m(\boldsymbol{x}, \boldsymbol{y})=$ $\alpha(\boldsymbol{y}) x_{i} \beta(\boldsymbol{y})$ :

$$
\begin{aligned}
D m(\boldsymbol{x}, \boldsymbol{y})[\boldsymbol{h}, 0] & =D \alpha(\boldsymbol{y})[0] x_{i} \beta(\boldsymbol{y})+\alpha(\boldsymbol{y}) h_{i} \beta(\boldsymbol{y})+\alpha(\boldsymbol{y}) x_{i} D \beta(\boldsymbol{y})[0] \\
& =m(\boldsymbol{h}, \boldsymbol{y}) .
\end{aligned}
$$

Recall $F_{g+i}(\boldsymbol{x}, \boldsymbol{y})=y_{g+i}$ for $1 \leq i \leq g$, hence $D F_{g+i}(\boldsymbol{x}, \boldsymbol{y})[\boldsymbol{h}, \boldsymbol{k}]=k_{i}$. In particular, if $X, Y, H, K \in M_{n}(\mathbb{C})^{g}$ then $D F(X, Y)[H, K]=0$ implies $K=0$. Thus $D p(Y)[H]=0$ if and only if $D F(X, Y)[H, K]=0$. Therefore, an application of Theorem A implies $p$ is bijective if and only if $F$ is bijective.

Lemma 4.11. Suppose $p$ is a bijective free polynomial with no constant term, $F$ is the scion of $p$, and $q$ and $G$ are the compositional inverses of $p$ and $F$ respectively. Let $\mathbb{G}(\boldsymbol{x}, \boldsymbol{y})[\boldsymbol{z}, \boldsymbol{w}]$ be the auxiliary inverse of $F$. Then,

(i) $\mathbb{G}(\boldsymbol{x}, \boldsymbol{y})[\boldsymbol{z}, \boldsymbol{y}]$ satisfies the conditions of Lemma 3.9 with $G(\boldsymbol{x}, \boldsymbol{y})$ as its unique solution;

(ii) $\mathbb{G}(\boldsymbol{x}, \boldsymbol{y})[\boldsymbol{z}, \boldsymbol{y}]$ is affine $\boldsymbol{z}$-linear;

(iii) $G(\boldsymbol{x}, \boldsymbol{y})=(D q(p(\boldsymbol{y}))[\boldsymbol{x}], \boldsymbol{y})$;

(iv) if $q$ is a free polynomial then $G$ is a free polynomial and

$$
\operatorname{deg}(q) \leq \operatorname{deg}(G) \leq \operatorname{deg}(p) \operatorname{deg}(q) .
$$

Proof. Since $\mathbb{G}$ is the compositional inverse of $F$, it automatically satisfies the conditions of Lemma 3.9. Hence, $\mathbb{G}(\boldsymbol{x}, \boldsymbol{y})[\boldsymbol{z}, \boldsymbol{w}]$ has $G(\boldsymbol{x}, \boldsymbol{y})$ as its unique solution.

Recall $F_{g+i}(\boldsymbol{x}, \boldsymbol{y})=y_{i}$ for $1 \leq i \leq g$. It follows that $\mathbb{G}_{g+i}(\boldsymbol{x}, \boldsymbol{y})[\boldsymbol{z}, \boldsymbol{w}]=y_{i}$ and thus $G_{g+i}(\boldsymbol{x}, \boldsymbol{y})=y_{i}$. In particular, $\mathbb{G}(\boldsymbol{x}, \boldsymbol{y})[\boldsymbol{z}, \boldsymbol{y}]$ still satisfies the conditions of Lemma 3.9 and $G(\boldsymbol{x}, \boldsymbol{y})$ is the unique solution of $\mathbb{G}(\boldsymbol{x}, \boldsymbol{y})[\boldsymbol{z}, \boldsymbol{y}]$. Thus, Lemma 4.1 applies to $\mathbb{G}(\boldsymbol{x}, \boldsymbol{y})[\boldsymbol{z}, \boldsymbol{y}]$ and $G(\boldsymbol{x}, \boldsymbol{y})$, justifying our use of $\mathbb{G}(\boldsymbol{x}, \boldsymbol{y})[\boldsymbol{z}, \boldsymbol{y}]$ in lieu of $\mathbb{G}(\boldsymbol{x}, \boldsymbol{y})[\boldsymbol{z}, \boldsymbol{w}]$.

To prove item (ii), let $J_{p}$ and $J_{F}$ be the Jacobian matrices of $p$ and $F$, respectively. Since both $p$ and $F$ have compositional inverses, Proposition 3.12 says $J_{p}^{-1}$ and $J_{F}^{-1}$ exist as matrices of formal power series. Hence,

$$
J_{F}^{-1}(\boldsymbol{z}, \boldsymbol{w})=\left(\begin{array}{cc}
J_{p}(\boldsymbol{w}) & 0 \\
D J_{p}(\boldsymbol{w})[\boldsymbol{z}] & I
\end{array}\right)^{-1}=\left(\begin{array}{cc}
J_{p}(\boldsymbol{w})^{-1} & 0 \\
-D J_{p}(\boldsymbol{w})[\boldsymbol{z}] J_{p}(\boldsymbol{w})^{-1} & I
\end{array}\right) .
$$


Observe $\boldsymbol{z}$ only appears in a free derivative, so $J_{F}^{-1}(\boldsymbol{z}, \boldsymbol{w})$ is affine $\boldsymbol{z}$-linear. Thus, $\mathbb{G}(\boldsymbol{x}, \boldsymbol{y})[\boldsymbol{z}, \boldsymbol{y}]=(\boldsymbol{x}, \boldsymbol{y}) J_{F}(\boldsymbol{z}, \boldsymbol{y})$ must also be affine $\boldsymbol{z}$-linear. Thus,

For (iii), since $(q \circ p)(\boldsymbol{x})=\boldsymbol{x}$, we have $\boldsymbol{x}=D(q \circ p)(\boldsymbol{y})[\boldsymbol{x}]=D q(p(\boldsymbol{y}))[D p(\boldsymbol{y})[\boldsymbol{x}]]$.

$$
(\boldsymbol{x}, \boldsymbol{y})=(D q(p(\boldsymbol{y}))[D p(\boldsymbol{y})[\boldsymbol{x}]], \boldsymbol{y})=(D q(p(\boldsymbol{y}))[F(\boldsymbol{x}, \boldsymbol{y})], \boldsymbol{y}) .
$$

Since $G_{g+i}(\boldsymbol{x}, \boldsymbol{y})=y_{i}$, substituting $G(\boldsymbol{x}, \boldsymbol{y})$ for $(\boldsymbol{x}, \boldsymbol{y})$ into (4.4) yields

$$
G(\boldsymbol{x}, \boldsymbol{y})=(D q(p(\boldsymbol{y}))[F(G(\boldsymbol{x}, \boldsymbol{y}))], \boldsymbol{y})=(D q(p(\boldsymbol{y}))[\boldsymbol{x}], \boldsymbol{y}) .
$$

Lastly, suppose $q$ is a free polynomial. It follows that $D q$ is a free polynomial, hence $G$ is a free polynomial. Since $\operatorname{deg}(q) \leq \operatorname{deg}(D q(p(\boldsymbol{y}))[\boldsymbol{x}]) \leq \operatorname{deg}(q) \operatorname{deg}(p)$ we conclude

$$
\operatorname{deg}(q) \leq \operatorname{deg}(G) \leq \operatorname{deg}(q) \operatorname{deg}(p)
$$

In a trivial sense, the degree bounds between $G$ and $q$ are not strict. If $p(\boldsymbol{x})=\boldsymbol{x}$ then $q(\boldsymbol{x})=\boldsymbol{x}, F(\boldsymbol{x}, \boldsymbol{y})=(\boldsymbol{x}, \boldsymbol{y})$ and $G(\boldsymbol{x}, \boldsymbol{y})=(\boldsymbol{x}, \boldsymbol{y})$. Hence, $\operatorname{deg}(q)=$ $\operatorname{deg}(q) \operatorname{deg}(p)=\operatorname{deg}(G)$.

Remark 4.12. We emphasize a point made in the proof of 4.11 ; since $G$ is the solution to both $\mathbb{G}(\boldsymbol{x}, \boldsymbol{y})[\boldsymbol{z}, \boldsymbol{y}]$ and $\mathbb{G}(\boldsymbol{x}, \boldsymbol{y})[\boldsymbol{z}, \boldsymbol{w}]$ we may use $\mathbb{G}(\boldsymbol{x}, \boldsymbol{y})[\boldsymbol{z}, \boldsymbol{y}]$ rather than $\mathbb{G}(\boldsymbol{x}, \boldsymbol{y})[\boldsymbol{z}, \boldsymbol{w}]$.

Example 4.13. Let

$$
p(\boldsymbol{x})=\left(x_{1}, x_{2}-x_{1}^{2}\right) \quad \text { and } \quad F(\boldsymbol{x}, \boldsymbol{y})=\left(x_{1}, x_{2}-x_{1} y_{1}-y_{1} x_{1}, y_{1}, y_{2}\right) .
$$

Hence

$$
\mathbb{I}(\boldsymbol{x})[\boldsymbol{z}]=\left(x_{1}, x_{2}+x_{1} z_{1}\right) \quad \text { and } \quad D \mathbb{I}(\boldsymbol{x}, \boldsymbol{z})[\boldsymbol{h}, \boldsymbol{k}]=\left(h_{1}, h_{2}+h_{1} z_{1}+x_{1} k_{1}\right)
$$

and $\mathbb{G}(\boldsymbol{x}, \boldsymbol{y})[\boldsymbol{z}, \boldsymbol{y}]=(D \mathbb{I}(p(\boldsymbol{y}), \boldsymbol{y})[\boldsymbol{x}, \boldsymbol{z}], \boldsymbol{y})=\left(x_{1}, x_{2}+x_{1} y_{1}+x_{1} z_{1}, y_{1}, y_{2}\right)$. Note $\mathrm{q}^{\circ 2}(\boldsymbol{x})[\boldsymbol{z}]=\left(x_{1}, x_{2}+x_{1}^{2}\right)=q(\boldsymbol{x})$ and $\mathbb{G}^{\circ 2}(\boldsymbol{x}, \boldsymbol{y})[\boldsymbol{z}, \boldsymbol{y}]=\left(x_{1}, x_{2}+x_{1} y_{1}+x_{1} x_{1}, y_{1}, y_{2}\right)$. Lastly, $2=\operatorname{deg}(q)=\operatorname{deg}(G)$, while $\operatorname{deg}(q) \operatorname{deg}(p)=4$, so $\operatorname{deg}(q)=\operatorname{deg}(G)<$ $\operatorname{deg}(q) \operatorname{deg}(p)$.

Proposition 4.10 tells us that a polynomial, $p$, is bijective if and only if its scion, $F$, is bijective. The scion is $\boldsymbol{x}$-affine linear, and its inverse function, $G$, is the unique algebraic solution to a proper algebraic polynomial that is $\boldsymbol{z}$-affine linear. We investigate precisely the formal power series that are generated by such $\boldsymbol{z}$-affine linear proper algebraic polynomials in Section 6 .

\section{Degree Bounds ON NC RATiOnal MAPS}

In order to prove Theorem 6.13 we require results about how rational functions behave when evaluated on matrices. Using rational degrees on nc rational functions, we prove Proposition 5.2, a result about the behavior of nc rational functions when they are evaluated on generic matrices. 
5.1. Rational degree bounds. In this subsection we introduce topics from noncommutative algebra in order to prove a general principle; evaluating a noncommutative rational function $r$ on a tuple of matrices produces a matrix whose entries behave similarly to $r$. A major obstacle in proving this principle is the fact that noncommutative rational functions cannot always be written as a fraction of polynomials. However, by introducing a commuting indeterminate $t$ we are able to characterize the degree of a nc rational function and its evaluations on matrices.

Definition 5.1. Suppose $U$ is a skew field containing $\mathbb{C}$ and $U[t]$ is the polynomial ring over $U$. We define the map $\operatorname{deg}_{t}: U[t] \rightarrow \mathbb{Z} \cup\{-\infty\}$ in the natural way; $\operatorname{deg}_{t}(0)=-\infty$ and if $r=r_{0}+r_{1} t+\cdots+r_{m} t^{m}, r_{m} \neq 0$, then $\operatorname{deg}_{t}(r)=m$. For any $r, s \in U[t]$,

(i) $\operatorname{deg}_{t}(r s)=\operatorname{deg}_{t}(r)+\operatorname{deg}_{t}(s)^{2}$,

(ii) $\operatorname{deg}_{t}(x+y) \leq \max \left\{\operatorname{deg}_{t}(x), \operatorname{deg}_{t}(y)\right\}$.

Theorem 2.1.15 in [MR01] tells us $U[t]=U \otimes \mathbb{C}[t]$ is an Ore domain with a classical ring of quotients, $U(t)$. Hence, for any $r \in U(t)$ there exist $\alpha, \beta \in U[t]$ such that $r=\alpha \beta^{-1}$.

We can uniquely extend $\operatorname{deg}_{t}$ to $U(t)$ (by Theorem 9.1 in [Coh95]) such that $\operatorname{deg}_{t}\left(r^{-1}\right)=-\operatorname{deg}_{t}(r)$, for all $r \neq 0$. In particular, if $r=\alpha \beta^{-1}$ then $\operatorname{deg}_{t}(r)=$ $\operatorname{deg}_{t}(\alpha)-\operatorname{deg}_{t}(\beta)$. We say $\operatorname{deg}_{t}: U(t) \rightarrow \mathbb{Z} \cup\{\infty\}$ is a rational degree map.

The main result of subsection 5.1 is as follows.

Proposition 5.2. Suppose $\mathbb{w}=\left\{w_{1}, \ldots, w_{h}\right\}$ is a collection of freely noncommuting indeterminates, $\boldsymbol{w}=\left(w_{1}, \ldots, w_{h}\right), t$ is a central indeterminate and $N \in \mathbb{Z}^{+}$. Let $r \in \mathbb{C} \nless \mathbb{w} \ngtr$ be a nonzero rational function such that $r($ tw $)=\alpha(\boldsymbol{w})[t] \beta(\boldsymbol{w})[t]^{-1}$, where $\alpha, \beta \in \mathbb{C} \nless \mathbb{w} \ngtr[t]$. Suppose

(i) $\mathbb{S}$ is a field containing $\mathbb{C}$,

(ii) $U \subset M_{N}(\mathbb{S})$ is a skew field generated by $u_{1}, \ldots, u_{h} \in M_{N}(\mathbb{S})$, each $u_{i} \neq 0$.

If $\operatorname{deg}_{t}$ is the rational degree map on $\mathbb{S}(t)$, then

$$
\operatorname{deg}_{t}\left(r\left(t u_{1}, \ldots, t u_{h}\right)_{i, j}\right) \leq \operatorname{deg}_{t}(\alpha(\boldsymbol{w})[t]) \in \mathbb{Z},
$$

for all $1 \leq i, j \leq N$, whenever $r\left(u_{1}, \ldots, u_{h}\right)$ is defined.

Of particular importance in Proposition 5.2 is that $\operatorname{deg}_{t}(\alpha(\boldsymbol{w})[t])$ is independent of $N$, hence it applies quite nicely to free functions.

Remark 5.3. The following definitions will be familiar to an algebraist but perhaps not to an analyst.

If $R$ is any commutative integral domain, then the field of fractions of $R$ is the smallest field in which $R$ can be embedded. Every integral domain has a field of fractions.

Next, a ring $D$ is said to be a noncommutative domain if it has no zero divisors, i.e. if $a, b \in D$ such that $a b=0$ then either $a=0$ or $b=0$. If, in addition, every nonzero element of $D$ has a multiplicative inverse then $D$ is said to be a skew field.

Let $R$ be a noncommutative domain and let $S$ be the set of all the nonzero elements of $R$. We say $R$ is a right Ore domain if for every $r \in R$ and $s \in S, r S \cap s R \neq \varnothing$. If $R$ is a right Ore domain, then there is a unique (up to $R$-isomorphism) skew field

\footnotetext{
${ }^{2}$ Item (i) is true as long as $U$ is a domain ( $U$ has no zero divisors). If $U$ has zero divisors then $\operatorname{deg}_{t}(r s) \leq \operatorname{deg}_{t}(r)+\operatorname{deg}_{t}(s)$.
} 
$D$ containing $R$ as a subring such that every element of $D$ has the form $r s^{-1}$, for $s, r \in R$ and $s \neq 0$. In this case, the skew field $D$ is called the classical ring of quotients of $R$, and it is unique up to isomorphism.

Lemma 5.4. Suppose $U$ is any skew field, $v \in U[t]^{n}$ is a row vector of polynomials and $M \in M_{n}(U(t))$ is an $n \times n$ matrix. If $\operatorname{deg}_{t}$ is a rational degree map on $U(t)$, then

$$
\operatorname{deg}_{t}\left((v M)_{i}\right) \leq \max _{k}\left\{\operatorname{deg}_{t}\left(v_{k}\right)\right\}+\max _{k}\left\{\operatorname{deg}_{t}\left(M_{k, i}\right)\right\},
$$

for each $1 \leq i \leq n$.

$$
\begin{aligned}
& \text { Moreover, if } \operatorname{deg}_{t}\left(v_{i}\right) \leq \delta \text { and } \operatorname{deg}_{t}\left(M_{i, j}\right) \leq \Delta \text { for } 1 \leq i, j \leq n \text { then } \\
& \max _{1 \leq k \leq n}\left\{\operatorname{deg}_{t}\left((v M)_{k}\right)\right\} \leq \delta+\Delta .
\end{aligned}
$$

Proof. Fix $1 \leq i \leq g$. Simply applying the properties of the degree map,

$$
\begin{aligned}
\operatorname{deg}_{t}\left((v M)_{i}\right) & =\operatorname{deg}_{t}\left(\sum_{k} v_{k} M_{k, i}\right) \leq \max _{k}\left\{\operatorname{deg}_{t}\left(v_{k} M_{k, i}\right)\right\} \\
& =\max _{k}\left\{\operatorname{deg}_{t}\left(v_{k}\right)+\operatorname{deg}_{t}\left(M_{k, i}\right)\right\} \\
& \leq \max _{k}\left\{\operatorname{deg}_{t}\left(v_{k}\right)\right\}+\max _{k}\left\{\operatorname{deg}_{t}\left(M_{k, i}\right)\right\} .
\end{aligned}
$$

Next, suppose $\operatorname{deg}_{t}\left(v_{i}\right) \leq \delta$ and $\operatorname{deg}_{t}\left(M_{i, j}\right) \leq \Delta$ for all $1 \leq i, j \leq n$. Thus, $\max _{k}\left\{\operatorname{deg}_{t}\left(v_{k}\right)\right\} \leq \delta$ and $\max _{i, k}\left\{\operatorname{deg}_{t}\left(M_{k, i}\right)\right\} \leq \Delta$. Finally, since $\operatorname{deg}_{t}\left((v M)_{i}\right) \leq$ $\delta+\Delta$ for $1 \leq i \leq n$, we conclude $\max _{i}\left\{\operatorname{deg}_{t}\left((v M)_{i}\right)\right\} \leq \delta+\Delta$.

Let $\mathrm{w}=\left\{w_{1}, \ldots, w_{h}\right\}$ be a finite collection of freely noncommuting indeterminates and let $\boldsymbol{w}=\left(w_{1}, \ldots, w_{h}\right)$. We recall a few facts about the construction of $\mathbb{C} \nless \mathbb{w} \ngtr$, the algebra of noncommutative rational functions. These results and definitions can be found in [KVV16] and [KV17].

Let $\mathcal{R}_{\mathbb{C}}(\mathbb{w})$ be the set of all noncommutative rational expressions over $\mathbb{C}$, i.e. all possible syntactically valid combinations of elements in $\mathbb{C}$ and $\mathbb{w}$, arithmetic operations (addition, multiplication, inversion) and parentheses. For example, $w_{1}+w_{1}, w_{1}\left(w_{2}-w_{1}\right)^{-1}$ and $0^{-1}$ are syntactically valid combinations. The inversion height of $\rho \in \mathcal{R}_{\mathbb{C}}(\mathbb{w})$ is the maximum number of nested inverses in $\rho$.

The subset of $M(\mathbb{C})^{h}$ at which $\rho$ is defined is denoted $\operatorname{dom} \rho$ and is called the domain of $\rho$. We say $\rho \in \mathcal{R}_{\mathbb{C}}(\mathbb{w})$ is nondegenerate if $\rho(A)$ is defined for some $A \in M(\mathbb{C})^{h}$. If $\rho_{1}, \rho_{2}$ are nondegenerate rational expressions then we say $\rho_{1} \sim \rho_{2}$ if and only if $\rho_{1}(A)=\rho_{2}(A)$ for all $A \in \operatorname{dom} \rho_{1} \cap \operatorname{dom} \rho_{2}$. This relation $\sim$ is an equivalence relation on the set of all nondegenerate rational expression in $\mathcal{R}_{\mathbb{C}}(\mathbb{w})$. We define $\mathbb{C} \nless \mathbb{w} \ngtr$, the skew field of noncommutative rational functions, to be the set of equivalence classes of nondegenerate expression with respect to $\sim$. If $r \in \mathbb{C} \nless \mathbb{w} \ngtr$, then the domain of $r$, denoted dom $r$, is defined as the union of the domains of all representatives of $r$ and if $A \in \operatorname{dom} r$ then $r(A)=\rho(A)$ for any representative $\rho \in \mathcal{R}_{\mathbb{C}}(\mathbb{w})$ such that $A \in \operatorname{dom} \rho$.

Remark 5.5. Both $\mathbb{C}\langle\mathbb{w}\rangle$ and $\mathbb{C}_{\text {rat }}\langle\langle\mathbb{w}\rangle\rangle$ embed into $\mathbb{C} \nless w \ngtr$, and in fact, if $r \in \mathbb{C} \nless u \ngtr$ is defined at 0 then $r \in \mathbb{C}\langle\langle\mathbb{w}\rangle\rangle$. Since every rational series (see Definition 2.1) is defined at 0 , we have that the rational series are exactly the nc rational functions defined at 0 .

We now introduce a lemma that will be implicitly used throughout the rest of Section 5 . 
Lemma 5.6. Suppose $r \in \mathbb{C} \nless \mathbb{w} \ngtr$. If $t$ is a central indeterminate, then $r($ tw $) \in$ $\mathbb{C} \nless \mathbb{w} \ngtr(t)$.

Proof. The proof follows quickly from induction on the inversion height.

Example 5.7. If $\rho$ is a rational function in commuting variables then $\rho$ can be written as a fraction of polynomials; $\rho=p q^{-1}$. Hence, it makes sense to talk about a rational degree map, $\operatorname{deg}(\rho)=\operatorname{deg}(p)-\operatorname{deg}(q)$.

In the noncommutative case we cannot guarantee that a rational function $r$ can be written as a fraction of polynomials. However, the commuting indeterminate $t$ we introduce acts as a yardstick for the rational degree of $r$. Since $\operatorname{deg}_{t}\left(q(t)^{-1}\right)=$ $-\operatorname{deg}_{t}(q(t))$ we can unpack a rational function by moving iteratively through the inversion heights.

For example, if

$$
r\left(x_{1}, x_{2}\right)=x_{1}\left(1-x_{1}\left(1-x_{2}\right)^{-1} x_{1}\right)^{-1}
$$

then we can guess $\operatorname{deg}\left(x_{1}\left(1-x_{2}\right)^{-1} x_{1}\right)=2-1, \operatorname{deg}\left(\left(1-x_{1}\left(1-x_{2}\right)^{-1} x_{1}\right)^{-1}\right)=1-2$ and, $\operatorname{deg}\left(x_{1}\left(1-x_{1}\left(1-x_{2}\right)^{-1} x_{1}\right)^{-1}\right)=2-2$. Introducing the commuting $t$ we get

$$
\begin{aligned}
r\left(t x_{1}, t x_{2}\right) & =t x_{1}\left(1-t x_{1}\left(1-t x_{2}\right)^{-1} t x_{1}\right)^{-1} \\
& =t x_{1} x_{1}^{-1}\left(x_{1}^{-1}-t^{2} x_{1}\left(1-t x_{2}\right)^{-1}\right)^{-1} \\
& =t\left(1-t x_{2}\right)\left(x_{1}^{-1}\left(1-t x_{2}\right)-t^{2} x_{1}\right)^{-1} \\
& =\left(t-t^{2} x_{2}\right)\left(x_{1}^{-1}-t x_{1}^{-1} x_{2}-t^{2} x_{1}\right)^{-1},
\end{aligned}
$$

a fraction of polynomials in $t$ with coefficients in $\mathbb{C} k \mathbb{x} \ngtr$. In fact, as we guessed, $\operatorname{deg}_{t}(r(t \boldsymbol{x}))=2-2=0$.

We are now in a position to prove Proposition 5.2.

Proposition 5.2. Suppose $\mathbb{w}=\left\{w_{1}, \ldots, w_{h}\right\}$ is a collection of freely noncommuting indeterminates, $\boldsymbol{w}=\left(w_{1}, \ldots, w_{h}\right), t$ is a central indeterminate and $N \in \mathbb{Z}^{+}$. Let $r \in \mathbb{C} \nless \mathbb{w} \ngtr$ be a nonzero rational function such that $r($ tw $)=\alpha(\boldsymbol{w})[t] \beta(\boldsymbol{w})[t]^{-1}$, where $\alpha, \beta \in \mathbb{C} \nless \mathbb{w} \ngtr[t]$. Suppose

(i) $\mathbb{S}$ is a field containing $\mathbb{C}$,

(ii) $U \subset M_{N}(\mathbb{S})$ is a skew field generated by $u_{1}, \ldots, u_{h} \in M_{N}(\mathbb{S})$, each $u_{i} \neq 0$. If $\operatorname{deg}_{t}$ is the rational degree map on $\mathbb{S}(t)$, then

$$
\operatorname{deg}_{t}\left(r\left(t u_{1}, \ldots, t u_{h}\right)_{i, j}\right) \leq \operatorname{deg}_{t}(\alpha(\boldsymbol{w})[t]) \in \mathbb{Z},
$$

for all $1 \leq i, j \leq N$, whenever $r\left(u_{1}, \ldots, u_{h}\right)$ is defined.

Proof. Suppose $\boldsymbol{u}=\left(u_{1}, \ldots, u_{h}\right)$ and $\kappa \in U[t]$ is a nonzero polynomial such that $\kappa(\boldsymbol{u})[t]=\sum_{j=1}^{m} k_{j}(\boldsymbol{u}) t^{j}$ with $k_{m}(\boldsymbol{u}) \neq 0$. Note

$$
\operatorname{deg}_{t}(\kappa(\boldsymbol{u})[t])=\max _{1 \leq i, j \leq N}\left\{\operatorname{deg}_{t}\left(\kappa(\boldsymbol{u})[t]_{i, j}\right)\right\}=m .
$$

We first show that $\operatorname{deg}_{t}(\operatorname{det}(\kappa(\boldsymbol{u})[t]))=N m$.

From the definition of the determinant, $\operatorname{deg}_{t}(\operatorname{det}(\kappa(\boldsymbol{u})[t])) \leq N m$. In fact, $\operatorname{det}(\kappa(\boldsymbol{u})[t])$ is a sum of products of $N$ entries of $\kappa(\boldsymbol{u})[t]$. Hence, $\operatorname{det}(\kappa(\boldsymbol{u})[t]) \in \mathbb{S}[t]$ and its $t^{N m}$ coefficient is exactly $\operatorname{det}\left(k_{m}(\boldsymbol{u})\right)$. Since $\mathbb{S}$ is a field, for any $A \in M_{N}(\mathbb{S})$, $\operatorname{det}(A) \neq 0$ if and only if $A$ is invertible. Next, $U$ being a skew field and $k_{m} \in U$ being nonzero imply $k_{m}$ is invertible, hence $\operatorname{det}\left(k_{m}\right) \neq 0$. Thus, $\operatorname{deg}_{t}(\operatorname{det}(\kappa(\boldsymbol{u})[t]))=N m$. That is,

$$
\operatorname{deg}_{t}(\operatorname{det}(\kappa(\boldsymbol{u})[t]))=N \operatorname{deg}_{t}(\kappa(\boldsymbol{u})[t]) .
$$


Next, we recall that the adjugate of any $N \times N$ matrix is a matrix of determinants of $(N-1) \times(N-1)$ sub-matrices. Hence, for all $1 \leq i, j \leq N$,

$$
\operatorname{deg}_{t}\left(\operatorname{adj}(\kappa(\boldsymbol{u})[t])_{i, j}\right) \leq(N-1) \operatorname{deg}_{t}(\kappa(\boldsymbol{u})[t]) .
$$

Since $\kappa(\boldsymbol{u})[t]^{-1}=\operatorname{det}(\kappa(\boldsymbol{u})[t])^{-1} \operatorname{adj}(\kappa(\boldsymbol{u})[t])$, Equations (5.1) and (5.2) imply

$$
\operatorname{deg}_{t}\left(\kappa(\boldsymbol{u})[t]^{-1}\right)=\max _{i, j}\left\{\operatorname{deg}_{t}\left(\frac{\operatorname{adj}(\kappa(\boldsymbol{u})[t])_{i, j}}{\operatorname{det}(\kappa(\boldsymbol{u})[t])}\right)\right\} \leq 0 .
$$

Next, $r(t \boldsymbol{w})=\alpha(\boldsymbol{w})[t] \beta(\boldsymbol{w})[t]^{-1}$ for some $\alpha, \beta \in \mathbb{C} \nless \mathbb{w} \ngtr[t]$ and assuming $r(\boldsymbol{u})$ is defined, $r(t \boldsymbol{u})=\alpha(\boldsymbol{u})[t] \beta(\boldsymbol{u})[t]^{-1}$. Hence,

$$
\begin{aligned}
\operatorname{deg}_{t}\left(r(t \boldsymbol{u})_{i, j}\right) & =\operatorname{deg}_{t}\left(\sum_{\ell=1}^{N} \alpha(\boldsymbol{u})[t]_{i, \ell}\left(\beta(\boldsymbol{u})[t]^{-1}\right)_{\ell, j}\right) \\
& \leq \max _{\ell}\left\{\operatorname{deg}_{t}\left(\alpha(\boldsymbol{u})[t]_{i, \ell}\left(\beta(\boldsymbol{u})[t]^{-1}\right)_{\ell, j}\right)\right\} \\
& \leq \max _{\ell}\left\{\operatorname{deg}_{t}\left(\alpha(\boldsymbol{u})[t]_{i, \ell}\right)\right\}+\max _{\ell}\left\{\operatorname{deg}_{t}\left(\left(\beta(\boldsymbol{u})[t]^{-1}\right)_{\ell, j}\right)\right\} \\
& \leq \operatorname{deg}_{t}(\alpha(\boldsymbol{u})[t])+\operatorname{deg}_{t}\left(\beta(\boldsymbol{u})[t]^{-1}\right) \\
& \leq \operatorname{deg}_{t}(\alpha(\boldsymbol{u})[t]) .
\end{aligned}
$$

Where the last inequality uses (5.3). Finally, $\operatorname{deg}_{t}(\alpha(\boldsymbol{u})[t]) \leq \operatorname{deg}_{t}(\alpha(\boldsymbol{w})[t])$ implies

$$
\operatorname{deg}_{t}\left(r(t \boldsymbol{u})_{i, j}\right) \leq \operatorname{deg}_{t}(\alpha(\boldsymbol{u})[t]) \leq \operatorname{deg}_{t}(\alpha(\boldsymbol{w})[t])
$$

for all $1 \leq i, j \leq N$.

It should be emphasized that (5.1) is not true for any polynomial in $M_{N}(\mathbb{S})[t]$. Rather, it holds true if and only if the leading coefficient is an invertible matrix. For example, if

$$
\lambda(t)=\left(\begin{array}{ll}
1 & 0 \\
0 & 1
\end{array}\right)+\left(\begin{array}{ll}
1 & 0 \\
0 & 0
\end{array}\right) t^{2}+\left(\begin{array}{ll}
0 & 1 \\
1 & 0
\end{array}\right) t^{3}+\left(\begin{array}{ll}
0 & 0 \\
0 & 1
\end{array}\right) t^{4}=\left(\begin{array}{cc}
1+t^{2} & t^{3} \\
t^{3} & 1+t^{4}
\end{array}\right)
$$

then $\operatorname{deg}_{t}(\lambda(t))=4$ while $\operatorname{deg}_{t}(\operatorname{det}(\lambda(t)))=\operatorname{deg}_{t}\left(1+t^{2}+t^{4}\right)=4<8$. On the other hand, if

$$
\lambda(t)=\left(\begin{array}{ll}
1 & 0 \\
0 & 1
\end{array}\right)+\left(\begin{array}{ll}
0 & 1 \\
1 & 0
\end{array}\right) t^{2}+\left(\begin{array}{ll}
1 & 0 \\
0 & 1
\end{array}\right) t^{4}=\left(\begin{array}{cc}
1+t^{4} & t^{2} \\
t^{2} & 1+t^{4}
\end{array}\right)
$$

then $\operatorname{deg}_{t}(\lambda(t))=4$ and $\operatorname{deg}_{t}(\operatorname{det}(\mu(t)))=\operatorname{deg}_{t}\left(1+t^{4}+t^{8}\right)=8$.

Proposition 5.2 gives credence to the notion that if $r$ is a noncommutative rational function, then $r(s)$ is a matrix of rational functions whose behavior is modeled by $r$. In particular we will apply this idea to generic matrix algebras.

5.2. Generic matrix algebras. Suppose $n \in \mathbb{Z}^{+}$. For each $i \in \mathbb{Z}^{+}, 1 \leq j \leq g$, and $1 \leq k, \ell \leq n$, let $\xi_{n, k, \ell}^{(i), j}$ be a commuting indeterminate. Next, for each $i \in \mathbb{Z}^{+}$, set $\xi_{n}^{(i)}=\left\{\xi_{n, k, \ell}^{(i), j}: 1 \leq j \leq g, 1 \leq k, \ell \leq n\right\}$. If $i, \hat{\imath} \in \mathbb{Z}^{+}$, then the algebras $\mathbb{C}\left[\xi_{n}^{(i)}\right]$ and $\mathbb{C}\left[\xi_{n}^{(i)} \cup \xi_{n}^{(\hat{\imath})}\right]$ have fields of fractions $\mathbb{C}\left(\xi_{n}^{(i)}\right)$ and $\mathbb{C}\left(\xi_{n}^{(i)} \cup \xi_{n}^{(\hat{\imath})}\right)$, respectively.

For $i, n \in \mathbb{Z}^{+}$and $1 \leq j \leq g$, define $\Xi_{n}^{(i), j}=\left(\xi_{n, k, \ell}^{(i), j}\right)_{k, \ell=1}^{n} \in M_{n}\left(\mathbb{C}\left[\xi_{n}^{(i)}\right]\right)$ to be a generic matrix of size $n$. Define $\operatorname{GM}_{n}\left(\Xi^{(i)}\right)$ to be the algebra of generic matrices; that is, the unital $\mathbb{C}$-subalgebra of $M_{n}\left(\mathbb{C}\left[\xi_{n}^{(i)}\right]\right)$ generated by $\Xi_{n}^{(i), 1}, \ldots, \Xi_{n}^{(i), g}$. Let

$$
\boldsymbol{\Xi}_{n}^{(i)}=\left(\Xi_{n}^{(i), 1}, \ldots, \Xi_{n}^{(i), g}\right)
$$


be a $g$-tuple of generic matrices.

To prepare for their use in Section 6 , we define $\operatorname{GM}_{n}\left(\left(\Xi^{(i)}\right)^{T}\right)$ to be the algebra of transposed generic matrices, that is, $\operatorname{GM}_{n}\left(\left(\Xi^{(i)}\right)^{T}\right)$ is the algebra generated by $\left(\Xi_{n}^{(i), 1}\right)^{T}, \ldots,\left(\Xi_{n}^{(i), g}\right)^{T}$. Let

$$
\left(\Xi_{n}^{(i)}\right)^{T}=\left(\left(\Xi_{n}^{(i), 1}\right)^{T}, \ldots,\left(\Xi_{n}^{(i), g}\right)^{T}\right)
$$

be a $g$-tuple of transposed generic matrices and let $\boldsymbol{\Xi}_{n}^{T,(j)}$ denote the $2 g$-tuple

$$
\left(\left(\Xi_{n}^{(j), 1}\right)^{T} \otimes I_{n}, \ldots,\left(\Xi_{n}^{(j), g}\right)^{T} \otimes I_{n}, I_{n} \otimes \Xi_{n}^{(j), 1}, \ldots, I_{n} \otimes \Xi_{n}^{(j), g}\right)
$$

where $\otimes$ is the Kronecker product. Lastly, let

$$
\operatorname{GM}_{n}\left(\Xi^{T,(j)}\right)=\operatorname{GM}_{n}\left(\left(\Xi^{(j)}\right)^{T}\right) \otimes \operatorname{GM}_{n}\left(\Xi^{(j)}\right)
$$

and observe $\mathrm{GM}_{n}\left(\Xi^{T,(j)}\right)$ is generated by $\left\{\left(\Xi_{n}^{(i)}\right)^{T} \otimes I_{n}\right\} \cup\left\{I_{n} \otimes \Xi_{n}^{(j)}\right\}$.

Remark 5.8. By Lemma 2.5 and Proposition 2.6 in [KVV16], $\mathrm{GM}_{n}\left(\Xi^{(i)}\right) \otimes \mathrm{GM}_{n}\left(\Xi^{(j)}\right)$ is contained in a skew field, $\mathrm{UD}_{n}\left(\Xi^{(i),(j)}\right)$. Since $\mathrm{GM}_{n}\left(\Xi^{(i)}\right) \otimes \mathrm{GM}_{n}\left(\Xi^{(j)}\right)$ and $\mathrm{GM}_{n}\left(\Xi^{T,(j)}\right)$ are isomorphic as algebras, $\mathrm{GM}_{n}\left(\Xi^{T,(j)}\right)$ must be contained in some skew field, $\mathrm{UD}_{n}\left(\Xi^{T,(j)}\right)$.

Thus, for any $n \in \mathbb{Z}^{+}$,

(i) $\mathbb{C}\left(\xi_{n}^{(1)}\right)$ is a field,

(ii) $\mathrm{GM}_{n}\left(\Xi^{T,(1)}\right) \subset M_{n^{2}}\left(\mathbb{C}\left(\xi_{n}^{(1)}\right)\right)$ is the $\mathbb{C}$-algebra generated by $\left\{\left(\Xi_{n}^{(1)}\right)^{T} \otimes\right.$ $\left.I_{n}\right\} \cup\left\{I_{n} \otimes \Xi_{n}^{(1)}\right\}$

(iii) $\mathrm{GM}_{n}\left(\Xi^{T,(1)}\right) \subset \mathrm{UD}_{n}\left(\Xi^{T,(1)}\right) \subset M_{n^{2}}\left(\mathbb{C}\left(\xi_{n}^{(1)}\right)\right)$ and $\mathrm{UD}_{n}\left(\Xi^{T,(1)}\right)$ is a skew field.

Hence, Proposition 5.2 is applicable; if $r \in \mathbb{C} \nless \mathbb{y} \cup \mathbb{x} \ngtr$, then there exists a $d_{r} \in \mathbb{Z}^{+}$ such that $\operatorname{deg}_{t}\left(r\left(t \boldsymbol{\Xi}_{n}^{T,(1)}\right)_{i, j}\right) \geq d_{r}$, for all $n \in \mathbb{Z}^{+}$and $1 \leq i, j \leq n^{2}$, whenever $r\left(\boldsymbol{\Xi}_{n}^{T,(1)}\right)$ is defined.

Example 5.9. By the Cayley-Hamilton theorem, any $X \in M_{2}(\mathbb{C})$ satisfies the relation $X^{2}=c_{1} X+c_{0} I_{2}$, for some scalars $c_{1}, c_{0} \in \mathbb{C}$. Take the commutator of both sides against $Y \in M_{2}(\mathbb{C})$,

$$
\left[X^{2}, Y\right]=c_{1}[X, Y]+c_{0}\left[I_{2}, Y\right]=c_{1}[X, Y]
$$

Next, take the commutator of both sides against $[X, Y]$,

$$
\left[\left[X^{2}, Y\right],[X, Y]\right]=c_{1}[[X, Y],[X, Y]]=0 .
$$

Thus, $p(y, x)=\left[\left[x^{2}, y\right],[x, y]\right]$ vanishes on $M_{2}(\mathbb{C})$, i.e. $p$ is a polynomial identity for $M_{2}(\mathbb{C})$. Let $r(y, x)=x^{2}(1-p(y, x))^{-1}$ and note $r$ is a fraction of the polynomials $x^{2}$ and $1-p(y, x)$. Thus, Proposition 5.2 implies $\operatorname{deg}_{t}\left(r\left(t \boldsymbol{\Xi}_{n}^{T,(1)}\right)_{i, j}\right) \leq \operatorname{deg}_{t}\left(t^{2} x^{2}\right)=2$, for all $n \in \mathbb{Z}^{+}$. In particular, if $n<3$ then $p\left(t \boldsymbol{\Xi}_{n}^{T,(1)}\right)=I_{2}$ and $\operatorname{deg}_{t}\left(r\left(t \boldsymbol{\Xi}_{n}^{T,(1)}\right)_{i, j}\right)=$ 2.

Lemma 5.10. Suppose $\eta: M(\mathbb{C})^{g} \rightarrow M(\mathbb{C})^{g}$ is a free analytic function with a power series that converges for each $X \in M(\mathbb{C})^{g}$ and $\eta\left(\boldsymbol{\Xi}_{n}^{(1)}\right)$ is a polynomial for each $n$. Define

$$
T=\left\{\operatorname{deg}_{t}\left(\eta_{j}\left(t \boldsymbol{\Xi}_{n}^{(1)}\right)_{k, \ell}\right): n \in \mathbb{Z}^{+}, 1 \leq j \leq g, 1 \leq k, \ell \leq n\right\} .
$$

If $T$ is bounded, then $\eta$ is a free polynomial.

Proof. This is Proposition 3.1 in [KŠ17]. 


\section{Hyporational SERIES}

Proposition 4.10 shows that if $p$ is a free polynomial, then $F(\boldsymbol{x}, \boldsymbol{y})=(D p(\boldsymbol{y})[\boldsymbol{x}], \boldsymbol{y})$ is a free polynomial, and $p$ is injective if and only if $F$ is injective. Lemma 4.11 implies $G$, the inverse of $F$, is the unique solution to a proper algebraic polynomial that is $\boldsymbol{z}$-affine linear. The $\boldsymbol{z}$-affine linearity is reminiscent of realizations of nc rational functions, see [Vol15] and [KV17]. With this similarity to realizations in mind, we generalize the class of rational series (see Definition 2.1) to a slightly larger class of formal power series that we call the hyporational series. In particular, the scion of a free polynomial has a hyporational series as its compositional inverse. We show that every rational series is hyporational and Theorem 6.13 , the main result of subsection 6.1 , says that a hyporational series without singularities is a free polynomial.

In subsection 6.3 , we apply the same techniques used to analyze hyporational series to the free derivative of polynomials. This leads to the construction of the hypo-Jacobian matrix of a free polynomial mapping and Theorem 6.18. Finally, combining Theorem 6.18 with results on automorphisms of $\mathbb{C}\langle\mathbb{x}\rangle$ proves the main result of this paper, Theorem 6.22 .

\subsection{Hyporealizations and hyporational series.}

Definition 6.1. Once more, suppose $\mathbb{Z}=\left\{z_{1}, \ldots, z_{h}\right\}$ is a set of freely noncommuting indeterminates where $h$ is not necessarily equal to $g$. Let a $\in(\mathbb{C}\langle\mathbb{x} \cup \mathbb{Z}\rangle)^{h}$. Recall from Definition 3.13 that a is a proper algebraic polynomial if a has no constant term and $\mathbb{d}_{z}(\mathrm{a})>1$, that is, if $w$ is a monomial appearing in a with $|w|_{z}>0$ then $|w| \geq 2$. We say a is $\boldsymbol{z}$-affine linear if $\mathbf{a}(\boldsymbol{x})[\boldsymbol{z}]=\mathfrak{a}(\boldsymbol{x})+\alpha(\boldsymbol{x})[\boldsymbol{z}]$, where $\alpha(\boldsymbol{x})[\boldsymbol{z}+\boldsymbol{w}]=\alpha(\boldsymbol{x})[\boldsymbol{z}]+\alpha(\boldsymbol{x})[\boldsymbol{w}]$. If a is both a proper algebraic polynomial and $\boldsymbol{z}$-affine linear then we say a is a hyporealization.

Suppose $r \in \mathbb{C}\langle\langle\mathbb{x}\rangle\rangle$ with constant term $r_{\mathbb{1}}$. We define $r$ to be a hyporational series if there exists a hyporealization a such that $r-r_{\mathbb{1}}$ is a component of the solution of a. Namely, there exists $\boldsymbol{r}=\left(\boldsymbol{r}_{1}, \ldots, \boldsymbol{r}_{h}\right) \in\left(\mathbb{C}\left\langle\langle\mathbb{x}\rangle_{+}\right)^{h}\right.$ such that $\mathrm{a}(\boldsymbol{x})[\boldsymbol{r}]=\boldsymbol{r}$ and $r-r_{\mathbb{1}}=\boldsymbol{r}_{i}$. Let $\mathbb{C}_{\text {hyp }}\langle\langle\mathbb{x}\rangle\rangle \subset \mathbb{C}\langle\langle\mathbb{x}\rangle\rangle$ denote the collection of all hyporational series.

Recall from Definition 2.1 that $\mathbb{C}_{\text {rat }}\langle\langle\mathbf{x}\rangle\rangle$ is the algebra of rational series.

Remark 6.2. We recall several facts from realization theory. Let $\mathbb{C} \nless \mathbf{x} \ngtr_{0} \subset \mathbb{C} \nless \mathbf{x} \ngtr$ denote the subring of nc rational functions that are regular at the origin:

$$
\mathbb{C} \nless \mathbb{x} \ngtr_{0}=\{r \in \mathbb{C} \nless \mathbb{x} \ngtr: 0 \in \operatorname{dom} r\} .
$$

As was mentioned in Remark 5.5, $\mathbb{C} \nless \mathbf{x}\rangle_{0}=\mathbb{C}_{\text {rat }}\langle\langle\mathbb{x}\rangle \text {. If } r \in \mathbb{C} \nless \mathbf{x}\rangle_{0}$, then $r$ has a realization; there exist $d \in \mathbb{Z}^{+}, c, b \in M_{d \times 1}(\mathbb{C})$ and $A_{1}, \ldots, A_{g} \in M_{d}(\mathbb{C})$ such that

$$
r=c^{T}\left(I_{d}-\sum_{j=1}^{g} A_{j} x_{j}\right)^{-1} b .
$$

Classical realization theory has a long and storied history in both mathematics and applied fields. We use definitions and results from [KV17], which provides an excellent exposition of realizations of nc rational functions and their domains. 
Remark 6.3. Every rational series is hyporational, that is, $\mathbb{C}_{\text {rat }}\langle\langle\mathbb{x}\rangle\rangle \subset \mathbb{C}_{\text {hyp }}\langle\langle\mathbf{x}\rangle\rangle$. We omit the proof of the above statement, however it follows readily from a rearrangement of the realization of a given nc rational map.

In fact, if $r$ is a formal power series with constant term $r_{\mathbb{1}}$, then $r \in \mathbb{C}_{\text {rat }}(\langle\langle\mathbf{x}\rangle\rangle)$ if and only if there exist $\mathscr{A} \in M_{h}\left(\mathbb{C}\langle\mathbb{x}\rangle_{+}\right)$and $\mathfrak{a} \in\left(\mathbb{C}\langle\mathbb{x}\rangle_{+}\right)^{h}$, such that $r-r_{\mathbb{1}}$ is a component of the solution to the hyporealization $\mathrm{a}(\boldsymbol{x})[\boldsymbol{z}]=\mathfrak{a}(\boldsymbol{x})+\boldsymbol{z} \mathscr{A}(\boldsymbol{x})$. This condition precisely delineates the difference between rational series and hyporational series that are not rational.

Realization theory tells us that there is a very intimate relationship between rational functions and linearity. Example 3.19 provides us with a function that is hyporational but not rational.

Example 6.4. The hyporealization $\$(\boldsymbol{x})[\boldsymbol{z}]=x_{2}+x_{1} z_{2} x_{1}$ has the solution $s(\boldsymbol{x})=$ $\sum_{n=0}^{\infty} x_{1}^{n} x_{2} x_{1}^{n}$. Thus $s$ is hyporational and $\$(\boldsymbol{x})[\boldsymbol{z}]$ is a hyporealization of $s$.

Arguing by contradiction, suppose $s$ is rational. Hence $s$ has a minimal representation $s(\boldsymbol{x})=c^{T}\left(I-A x_{1}-B x_{2}\right)^{-1} b$, where $A, B \in M_{n}(\mathbb{C})$ and $b, c \in M_{n \times 1}(\mathbb{C})^{g}$. Let $m_{A}(t)=a_{0}+a_{1} t+\cdots+a_{d} t^{d}$ be the minimal polynomial of $A$ and note there is a $k$ so that $a_{k} \neq 0$ since $A \neq 0$. Observe $c^{T} A^{i} B A^{j} b=\delta(i, j)$ where $\delta$ is the Kronecker delta. Hence

$$
\begin{aligned}
0 & =c^{T} 0 b=c^{T} \overline{a_{k}} m_{A}(A) B A^{k} b=\sum_{i=0}^{d} a_{i} \overline{a_{k}} c^{T}\left(A^{i} B A^{k}\right) b \\
& =\left|a_{k}\right|^{2} c^{T}\left(A^{k} B A^{k}\right) b=\left|a_{k}\right|^{2} \\
& >0
\end{aligned}
$$

a contradiction. Therefore, $s$ is not rational.

One of the main advantages of the realization theory of nc rational functions is that the intrinsic linearity of rationals is expressed through matrices. Since hyporational series are generated by linear proper algebraic polynomials, we would like to imitate rational realization theory for hyporational series. This is precisely what we do in subsection 6.2 .

\subsection{Hypomatrix representations.}

Definition 6.5. For any $\mathbb{C}$-algebra $R$ and $n \in \mathbb{Z}^{+}$, the map vec $[n]: M_{n}(R) \rightarrow$ $M_{1 \times n^{2}}(R)$ given by

$$
\operatorname{vec}[n](A)_{(i-1) n+j}=A_{i, j}
$$

is a linear isomorphism taking an $n \times n$ matrix with entries in $R$ to a length $n^{2}$ row vector with entries in $R$.

If $U, V \in M_{n}(R)$ then $U \otimes V \in M_{n^{2}}(R)$ is the standard Kronecker product. Furthermore, if $Z, U, V \in M_{n}(R)$ then the product $\operatorname{vec}[n](Z) \cdot(U \otimes V)$ is the product of a $1 \times n^{2}$ row vector and an $n^{2} \times n^{2}$ matrix.

Let us see $\operatorname{vec}[n]$ in action. If $A \in M_{3}\left(\mathbb{C}\left[x_{1}, \ldots, x_{9}\right]\right)$ with

$$
A=\left(\begin{array}{lll}
x_{1} & x_{2} & x_{3} \\
x_{4} & x_{5} & x_{6} \\
x_{7} & x_{8} & x_{9}
\end{array}\right),
$$

then $\operatorname{vec}[3](A)=\left(x_{1}, \quad x_{2}, \quad x_{3}, \quad x_{4}, \quad x_{5}, \quad x_{6}, \quad x_{7}, \quad x_{8}, \quad x_{9}\right)$. 
For notational convenience, we allow $\operatorname{vec}[n]$ to apply coordinate-wise to tuples:

$$
\operatorname{vec}[n]\left(\left(A_{1}, \ldots, A_{m}\right)\right)=\left(\operatorname{vec}[n]\left(A_{1}\right), \ldots, \operatorname{vec}[n]\left(A_{m}\right)\right) .
$$

Lemma 6.6. If $U, V, Z \in M_{n}(R)$ then

$$
\operatorname{vec}[n](U Z V)=\operatorname{vec}[n](Z) \cdot\left(U^{T} \otimes V\right) \in M_{1 \times n^{2}}(R) .
$$

Proof. Our definition of $\mathbf{v e c}[n]$ is a left sided version of the vec function defined at 4.2.9 in [HJ94]. By adapting Lemma 4.3.1 in [HJ94] we conclude vec $[n](U Z V)=$ $\operatorname{vec}[n](Z) \cdot\left(U^{T} \otimes V\right)$.

Recall

$$
\Xi_{n}^{(1)}=\left(\Xi_{n}^{(1), 1}, \ldots, \Xi_{n}^{(1), g}\right)
$$

is a $g$-tuple of generic matrices while

$$
\Xi_{n}^{T,(1)}=\left(\left(\Xi_{n}^{(1), 1}\right)^{T} \otimes I_{n}, \ldots,\left(\Xi_{n}^{(1), g}\right)^{T} \otimes I_{n}, I_{n} \otimes \Xi_{n}^{(1), 1}, \ldots, I_{n} \otimes \Xi_{n}^{(1), g}\right),
$$

is a $2 g$-tuple of $n^{2}$-matrices over $\mathbb{C}\left[\xi^{(i)}\right]$.

Borrowing from [KVV16], we define

$$
\begin{aligned}
\mathbb{C}\langle y \leftrightarrow \mathbb{x}\rangle: & =\mathbb{C}\langle\mathbb{y}\rangle \otimes \mathbb{C}\langle\mathbb{x}\rangle \\
& \cong \mathbb{C}\langle\mathbb{y} \cup \mathbb{x}\rangle /\left(\left[y_{i}, x_{j}\right]: 1 \leq i, j \leq g\right),
\end{aligned}
$$

to be the bipartite free $\mathbb{C}$-algebra. The algebra $\mathbb{C}\langle y \leftrightarrow \mathbb{X}\rangle$ is contained in a skew field of fractions, $\mathbb{C} \nless y \leftrightarrow \mathbb{x} \ngtr$, the bipartite rational functions.

Remark 6.7. We briefly define the transpose of a polynomial. For any $w \in\langle\mathbb{x}\rangle$ with $w=x_{i_{1}} x_{i_{2}} \ldots x_{i_{n}}$, we say $w^{T}=x_{i_{n}} x_{i_{n-1}} \ldots x_{i_{1}}$. Hence, for any polynomial $p=\sum p_{w} w$, we define $p^{T}=\sum p_{w} w^{T}$. In particular, if $X=\left(X_{1}, \ldots, X_{g}\right)$ is a tuple of matrices, then $p^{T}\left(X^{T}\right)=(p(X))^{T}$.

Proposition 6.8. Suppose $\mathrm{a} \in(\mathbb{C}\langle\mathbb{x} \cup \mathbb{Z}\rangle)^{g}$ is a hyporealization with $\mathrm{a}(\boldsymbol{x})[\boldsymbol{z}]=\mathfrak{a}(\boldsymbol{x})+$ $\alpha(\boldsymbol{x})[\boldsymbol{z}]$. If $a \in\left(\mathbb{C}\left\langle\langle\mathbb{x}\rangle_{+}\right)^{g}\right.$ is the hyporational series such that $\mathbf{a}(\boldsymbol{x})[a(\boldsymbol{x})]=a(\boldsymbol{x})$, then there exists $\Phi \in M_{g}(\mathbb{C}\langle y \leftrightarrow \mathbb{x}\rangle)$ such that

(i) $\operatorname{vec}[n](\mathfrak{a}(X)[Z])=\operatorname{vec}[n](\mathfrak{a}(X))+\operatorname{vec}[n](Z) \cdot \Phi\left(X^{T} \otimes I, I \otimes X\right)$, for all $n$ and $X, Z \in M_{n}(\mathbb{C})^{g}$;

(ii) $(I-\Phi(\boldsymbol{y}, \boldsymbol{x}))$ is invertible as a matrix over $\mathbb{C} \nless \mathrm{y} \leftrightarrow \mathbb{\mathbb { X }} \ngtr$;

(iii) $\operatorname{dom}_{n m}\left((I-\Phi)_{i, j}^{-1}\right) \neq \varnothing$ for all $n, m \in \mathbb{Z}^{+}$and $1 \leq i, j \leq g$;

(iv) $\left(I-\Phi\left(\boldsymbol{\Xi}_{n}^{T,(1)}\right)\right)^{-1}$ is defined for all $n \in \mathbb{Z}^{+}$;

(v) $\operatorname{vec}[n]\left(a\left(\boldsymbol{\Xi}_{n}^{(1)}\right)\right)=\operatorname{vec}[n]\left(\mathfrak{a}\left(\boldsymbol{\Xi}_{n}^{(1)}\right)\right) \cdot\left(I-\Phi\left(\boldsymbol{\Xi}_{n}^{T,(1)}\right)\right)^{-1}$, for all $n \in \mathbb{Z}^{+}$.

Proof. For $1 \leq i \leq g$, we write

$$
\alpha^{i}(\boldsymbol{x})[\boldsymbol{z}]=\sum_{\ell} \sum_{j=1}^{g} U_{i, j}^{\ell}(\boldsymbol{x}) z_{j} V_{i, j}^{\ell}(\boldsymbol{x}),
$$

where each $U_{i, j}, V_{i, j} \in \mathbb{C}\langle\mathbb{x}\rangle$. Set $\Phi_{j, i}(\boldsymbol{y}, \boldsymbol{x})=\sum_{\ell}\left(U_{i, j}^{\ell}\right)^{T}(\boldsymbol{y}) \otimes V_{i, j}^{\ell}(\boldsymbol{x})$ and note

$$
\begin{aligned}
\Phi\left(X^{T} \otimes I, I \otimes X\right) & =\left(\Phi_{j, i}\left(X^{T} \otimes I, I \otimes X\right)\right)_{j, i=1}^{g} \\
& =\left(\sum_{\ell}\left(U_{i, j}^{\ell}\right)^{T}\left(X^{T} \otimes I\right) V_{i, j}^{\ell}(I \otimes X)\right)_{j, i=1}^{g},
\end{aligned}
$$


for all $X \in M(\mathbb{C})^{g}$. Let $1 \leq i \leq g$,

$$
\begin{aligned}
\operatorname{vec}[n]\left(\alpha^{i}(X)[Z]\right) & =\operatorname{vec}[n]\left(\sum_{\ell} \sum_{j=1}^{g} U_{i, j}^{\ell}(X) Z_{j} V_{i, j}^{\ell}(X)\right) \\
& =\sum_{\ell} \sum_{j=1}^{g} \operatorname{vec}[n]\left(U_{i, j}^{\ell}(X) Z_{j} V_{i, j}^{\ell}(X)\right) \\
& =\sum_{j=1}^{g} \sum_{\ell} \operatorname{vec}[n]\left(Z_{j}\right) \cdot\left(U_{i, j}^{\ell}(X)^{T} \otimes V_{i, j}^{\ell}(X)\right),
\end{aligned}
$$

where the last equality is using Lemma 6.6. Continuing on,

$$
\begin{aligned}
\operatorname{vec}[n]\left(\alpha^{i}(X)[Z]\right) & =\sum_{j=1}^{g} \sum_{\ell} \operatorname{vec}[n]\left(Z_{j}\right) \cdot\left(U_{i, j}^{\ell}(X)^{T} \otimes V_{i, j}^{\ell}(X)\right) \\
& =\sum_{j=1}^{g} \operatorname{vec}[n]\left(Z_{j}\right) \sum_{\ell}\left(\left(U_{i, j}^{\ell}\right)^{T}\left(X^{T}\right) \otimes V_{i, j}^{\ell}(X)\right) \\
& =\sum_{j=1}^{g} \operatorname{vec}[n]\left(Z_{j}\right) \sum_{\ell}\left(\left(U_{i, j}^{\ell}\right)^{T}\left(X^{T} \otimes I\right) V_{i, j}^{\ell}(I \otimes X)\right) \\
& =\sum_{j=1}^{g} \operatorname{vec}[n]\left(Z_{j}\right) \sum_{j=1}^{g} \Phi_{j, i}\left(X^{T} \otimes I, I \otimes X\right) \\
& =\left(\operatorname{vec}[n](Z) \cdot \Phi\left(X^{T} \otimes I, I \otimes X\right)\right)_{i} .
\end{aligned}
$$

Therefore, $\operatorname{vec}[n](\alpha(X)[Z])=\operatorname{vec}[n](Z) \Phi\left(X^{T} \otimes I, I \otimes X\right)$ and

$$
\operatorname{vec}[n](\mathfrak{a}(X)[Z])=\operatorname{vec}[n](\mathfrak{a}(\boldsymbol{x}))+\operatorname{vec}[n](Z) \cdot \Phi\left(X^{T} \otimes I, I \otimes X\right)
$$

for all $n$ and $X, Z \in M_{n}(\mathbb{C})^{g}$. Thus, item (i) is proved.

For item (ii), note $I-\Phi(\boldsymbol{y}, \boldsymbol{x}) \in M_{g}(\mathbb{C}\langle\mathrm{y} \leftrightarrow \mathbb{x}\rangle)$, hence $I-\Phi(\boldsymbol{y}, \boldsymbol{x}) \in M_{g}(\mathbb{C} \nless \mathrm{y} \leftrightarrow \mathbb{X} \ngtr)$. Since $I-\Phi(0,0)=I$, is invertible, Proposition 3.8 in [KVV16] implies $I-\Phi$ is invertible and $(I-\Phi(\boldsymbol{y}, \boldsymbol{x}))^{-1} \in M_{g}(\mathbb{C} \nless \mathrm{y} \leftrightarrow \mathbb{\mathbb { X }} \ngtr)$.

For item (iii) we note $\left(0_{n} \otimes I_{m}, I_{n} \otimes 0_{m}\right)=\left(0_{n m}, 0_{n m}\right) \in \operatorname{dom}_{n m}\left((I-\Phi)_{i, j}^{-1}\right)$ since $I_{n m}-\Phi\left(0_{n m}, 0_{n m}\right)=I_{n m}$ is invertible.

Item (iv) is simply a consequence of (iii);

$$
\operatorname{dom}_{n^{2}}\left((I-\Phi)_{i, j}^{-1}\right) \subset \operatorname{dom}\left(\left(I-\Phi\left(\boldsymbol{\Xi}_{n}^{T,(1)}\right)\right)_{i, j}^{-1}\right) .
$$

Finally, substitute $\boldsymbol{\Xi}_{n}^{(1)}$ in for $X$ and $a\left(\boldsymbol{\Xi}_{n}^{(1)}\right)$ in for $Z$ in (6.1) to get

$$
\begin{aligned}
\operatorname{vec}[n]\left(a\left(\boldsymbol{\Xi}_{n}^{(1)}\right)\right) & =\operatorname{vec}[n]\left(\mathfrak{a}\left(\boldsymbol{\Xi}_{n}^{(1)}\right)\left[a\left(\boldsymbol{\Xi}_{n}^{(1)}\right)\right]\right) \\
& =\operatorname{vec}[n]\left(\mathfrak{a}\left(\boldsymbol{\Xi}_{n}^{(1)}\right)\right)+\operatorname{vec}[n]\left(a\left(\boldsymbol{\Xi}_{n}^{(1)}\right)\right) \cdot \Phi\left(\boldsymbol{\Xi}_{n}^{T,(1)}\right) .
\end{aligned}
$$

Hence by rearranging,

$$
\operatorname{vec}[n]\left(a\left(\boldsymbol{\Xi}_{n}^{(1)}\right)\right) \cdot\left(I_{g n^{2}}-\Phi\left(\boldsymbol{\Xi}_{n}^{T,(1)}\right)\right)=\operatorname{vec}[n]\left(\mathfrak{a}\left(\boldsymbol{\Xi}_{n}^{(1)}\right)\right) .
$$

Multiplying both sides on the right by $\left(I_{g n^{2}}-\Phi\left(\boldsymbol{\Xi}_{n}^{T,(1)}\right)\right)^{-1}$ yields

$$
\boldsymbol{v e c}[n]\left(a\left(\boldsymbol{\Xi}_{n}^{(1)}\right)\right)=\operatorname{vec}[n]\left(\mathfrak{a}\left(\boldsymbol{\Xi}_{n}^{(1)}\right)\right) \cdot\left(I_{g n^{2}}-\Phi\left(\boldsymbol{\Xi}_{n}^{T,(1)}\right)\right)^{-1},
$$

as desired. 
If $a$ is a hyporational series then $a[n]=\left.a\right|_{M_{n}(\mathbb{C})^{g}}$ is a rational function in $g n^{2}$ commuting indeterminates by Proposition 6.8(v). In fact, Proposition 6.8 shows that a small amount of commutativity is all the prevents a hyporational from being rational.

Definition 6.9. Suppose $\mathfrak{a}(\boldsymbol{x})[\boldsymbol{z}]=\mathfrak{a}(\boldsymbol{x})+\alpha(\boldsymbol{x})[\boldsymbol{z}]$ is hyporealization. Let $\Phi \in$ $M_{g}(\mathbb{C}\langle\mathbb{y} \leftrightarrow \mathbb{x}\rangle)$ be the matrix constructed in Proposition 6.8. We define $\Phi$ to be the hypomatrix representation of a. That is,

$$
\operatorname{vec}[n](\mathfrak{a}(X)[Z])=\operatorname{vec}[n](\mathfrak{a}(X))+\operatorname{vec}[n](Z) \cdot\left(I-\Phi\left(X^{T} \otimes I, I \otimes X\right)\right)
$$

for all $X, Z \in M_{n}(\mathbb{C})^{g}$ and $n \in \mathbb{Z}^{+}$.

Let $a \in \mathbb{C}_{\text {hyp }}\langle\langle\mathbb{x}\rangle\rangle$ be hyporational. Define

$$
\operatorname{dom}_{n}(a)=\bigcup_{\Phi \in \mathscr{A}} \operatorname{dom}_{n}\left((I-\Phi)^{-1}\right),
$$

where $\mathscr{A}$ is the collection of all hypomatrix representations of $a$.

Suppose $X \in \operatorname{dom}_{n}(a)$. Let $\mathfrak{a}(\boldsymbol{x})[\boldsymbol{z}]=\mathfrak{a}(\boldsymbol{x})+\alpha(\boldsymbol{x})[\boldsymbol{z}]$ be a hyporealization such that $a$ is the first component of the solution of a and $X \in \operatorname{dom}_{n}\left((I-\Phi)^{-1}\right)$, where $\Phi$ is the associated hypomatrix representation of a. We define

$$
a(X)=\left(\operatorname{vec}[n]^{-1}\left(\operatorname{vec}[n](\mathfrak{a}(X)) \cdot\left(I-\Phi\left(X^{T} \otimes I, I \otimes X\right)\right)^{-1}\right)\right)_{1} .
$$

Thus, we can evaluate $a$ at any $X \in \operatorname{dom}_{n}(a)$.

Example 6.10. Let $p\left(x_{1}, x_{2}, x_{3}\right)=\left(x_{1}, x_{2}-x_{1}^{2}, x_{3}+x_{1}\left(-x_{2}+x_{1} x_{2}\right)-x_{2}^{2}\right)$, hence

$$
p(\boldsymbol{x})=\boldsymbol{x}\left(\begin{array}{ccc}
1 & -x_{1} & -x_{2}+x_{1} x_{2} \\
0 & 1 & -x_{2} \\
0 & 0 & 1
\end{array}\right)=\boldsymbol{x}\left(\begin{array}{ccc}
1 & x_{1} & x_{2} \\
0 & 1 & x_{2} \\
0 & 0 & 1
\end{array}\right)^{-1}
$$

and $\mathrm{q}(\boldsymbol{x})[\boldsymbol{z}]=\left(x_{1}, x_{2}+x_{1} z_{1}, x_{3}+x_{1} z_{2}+x_{2} z_{2}\right)$. Note $\mathrm{q}$ is a hyporealization and setting

$$
\Phi(\boldsymbol{y}, \boldsymbol{x})=\left(\begin{array}{ccc}
0 & y_{1} \otimes 1 & 0 \\
0 & 0 & \left(y_{1}+y_{2}\right) \otimes 1 \\
0 & 0 & 0
\end{array}\right),
$$

$\Phi$ is the hypomatrix representation of q. Observe $\Phi$ is nilpotent, and

$$
(I-\Phi(\boldsymbol{y}, \boldsymbol{x}))^{-1}=\left(\begin{array}{ccc}
1 & y_{1} \otimes 1 & y_{1}\left(y_{1}+y_{2}\right) \otimes 1 \\
0 & 1 & \left(y_{1}+y_{2}\right) \otimes 1 \\
0 & 0 & 1
\end{array}\right),
$$

and

$$
\operatorname{vec}[n]\left(\boldsymbol{\Xi}_{n}^{(1)}\right)\left(I-\Phi\left(\boldsymbol{\Xi}_{n}^{T,(1)}\right)\right)^{-1}=\operatorname{vec}[n]\left(q\left(\boldsymbol{\Xi}_{n}^{(1)}\right)\right) .
$$

Thus $q(\boldsymbol{x})=\left(x_{1}, x_{2}+x_{1}^{2}, x_{3}+\left(x_{1}+x_{2}\right) x_{2}+\left(x_{1}+x_{2}\right) x_{1}^{2}\right)$.

Example 6.11. We once again revisit Example 3.19. That is, $p(\boldsymbol{x})=\left(x_{1}, x_{2}-\right.$ $\left.x_{1} x_{2} x_{1}\right), q(\boldsymbol{x})=\left(x_{1}, \sum_{n=0}^{\infty} x_{1}^{n} x_{2} x_{1}^{n}\right)$ and the auxiliary inverse of $p$ is $\mathbb{q}(\boldsymbol{x})[\boldsymbol{z}]=$ $\left(x_{1}, x_{2}+x_{1} z_{2} z_{1}\right)$. Since $q^{1}(\boldsymbol{x})=x_{1}, \hat{\mathrm{q}}(\boldsymbol{x})[\boldsymbol{z}]=\left(x_{1}, \quad x_{2}+x_{1} z_{2} x_{1}\right)$ is a hyporealization with $\hat{\mathbb{q}}(\boldsymbol{x})[q(\boldsymbol{x})]=q(\boldsymbol{x})$. The hypomatrix representation of $\hat{\mathrm{q}}$ is

$$
I-\Phi(\boldsymbol{y}, \boldsymbol{x})=\left(\begin{array}{cc}
1 \otimes 1 & 0 \\
0 & 1 \otimes 1-y_{1} \otimes x_{1}
\end{array}\right)
$$


with inverse,

$$
(I-\Phi(\boldsymbol{y}, \boldsymbol{x}))^{-1}=\left(\begin{array}{cc}
1 \otimes 1 & 0 \\
0 & \left(1 \otimes 1-y_{1} \otimes x_{1}\right)^{-1}
\end{array}\right) .
$$

Recall that the inverse of $J_{p}$, the Jacobian matrix of $p$, is a polynomial matrix, however $p$ is not injective. In this case, the hypomatrix representation witnesses the non-injectivity of $p$ since $(I-\Phi)^{-1}$ is not a polynomial matrix.

Recall (see Lemma 5.6) that if $r \in \mathbb{C} \nless \mathbb{x} \ngtr$ then $r(t \boldsymbol{x}) \in \mathbb{C} \nless \mathbb{x} \ngtr(t)$. Since $\mathbb{C} \nless \mathbb{x} \ngtr$ is a skew field, $\mathbb{C} \nless \mathbb{x} \ngtr(t)$ is the classical ring of quotients of $\mathbb{C} \nless \mathbb{x} \ngtr[t]$. Hence there exist $\alpha, \beta \in \mathbb{C} \nless \mathbb{x} \ngtr[t]$ such that $r(t \boldsymbol{x})=\alpha(\boldsymbol{x})[t] \beta(\boldsymbol{x})[t]^{-1}$ and we can extend $\operatorname{deg}_{t}$ to $\mathbb{C} \nless \mathbb{x} \ngtr(t)$.

Lemma 6.12. If $a \in(\mathbb{C}\langle(\mathbb{\mathbb { x }}\rangle))^{g}$ is a tuple of hyporational series then there exists $\Delta \in \mathbb{Z}$ such that for all $n \in \mathbb{Z}^{+}$,

$$
\max \left\{\operatorname{deg}_{t}\left(a_{k}\left(t \boldsymbol{\Xi}_{n}^{(1)}\right)_{i, j}\right): 1 \leq k \leq g, 1 \leq i, j \leq n\right\} \leq \Delta .
$$

Proof. Let $\mathfrak{a}(\boldsymbol{x})[\boldsymbol{z}]=\mathfrak{a}(\boldsymbol{x})+\alpha(\boldsymbol{x})[\boldsymbol{z}]$ be a hyporealization of $a$ and let $\Phi$ be the hypomatrix representation of $\mathfrak{a}$. We begin by noting that $\mathfrak{a}$ is a free polynomial, hence for $1 \leq i, j \leq n$ and $1 \leq k \leq g, \operatorname{deg}_{t}\left(\mathfrak{a}_{k}\left(t \boldsymbol{\Xi}_{n}^{(1)}\right)_{i, j}\right) \leq \operatorname{deg}_{t}\left(\mathfrak{a}_{k}(t \boldsymbol{x})\right)$. Set $\delta=\max _{k}\left\{\operatorname{deg}_{t}\left(\mathfrak{a}_{k}(t \boldsymbol{x})\right)\right\}$. Since $\mathbf{v e c}[n]$ preserves the entries of matrices,

$$
\begin{aligned}
\max _{1 \leq \ell \leq g n^{2}}\left\{\operatorname{deg}_{t}\left(\operatorname{vec}[n]\left(\mathfrak{a}\left(t \boldsymbol{\Xi}_{n}^{(1)}\right)\right)_{\ell}\right)\right\} & =\max _{\substack{1 \leq k \leq g \\
1 \leq i, j \leq n}}\left\{\operatorname{deg}_{t}\left(\mathfrak{a}_{k}\left(t \boldsymbol{\Xi}_{n}^{(1)}\right)_{i, j}\right)\right\} \\
& \leq \max _{1 \leq k \leq g}\left\{\operatorname{deg}_{t}\left(\mathfrak{a}_{k}(t \boldsymbol{x})\right)\right\}=\delta .
\end{aligned}
$$

Next we recall $(I-\Phi)^{-1} \in M_{g}(\mathbb{C} \nless \mathbb{y} \cup \mathbb{x} \ngtr)$ and $\left(I-\Phi\left(t \boldsymbol{\Xi}_{n}^{T,(1)}\right)\right)^{-1}$ exists for all $n \in \mathbb{Z}^{+}$. For notational ease we set $\Psi=(I-\Phi)^{-1}$. Each $\Psi_{i, j}(t \boldsymbol{y}, t \boldsymbol{x}) \in \mathbb{C} \nless \mathbb{y} \leftrightarrow \mathbb{X} \ngtr(t)$, thus by Proposition 5.2 we know there exists $d_{i, j} \in \mathbb{Z}^{+}$such that for all $n \in \mathbb{Z}^{+}$, $\operatorname{deg}_{t}\left(\Psi_{i, j}\left(t \boldsymbol{\Xi}_{n}^{T,(1)}\right)\right) \leq d_{i, j}$. If we set $D=\max _{i, j}\left\{d_{i, j}\right\}$, then for all $n \in \mathbb{Z}^{+}$,

$$
\max _{1 \leq k, \ell \leq g n^{2}}\left\{\operatorname{deg}_{t}\left(\Psi\left(t \boldsymbol{\Xi}_{n}^{T,(1)}\right)_{k, \ell}\right)\right\} \leq D \text {. }
$$

Proposition 6.8 says for each $n \in \mathbb{Z}^{+}, \operatorname{vec}[n]\left(a\left(t \boldsymbol{\Xi}_{n}^{(1)}\right)\right)$ is the product of the row vector $\operatorname{vec}[n]\left(\mathfrak{a}\left(t \boldsymbol{\Xi}_{n}^{(1)}\right)\right)$ and the matrix $\Psi\left(t \boldsymbol{\Xi}_{n}^{T,(1)}\right)$. Thus, in light of the degree bounds found above and Lemma 5.4, we have

$$
\max _{1 \leq k \leq g n^{2}}\left\{\operatorname{deg}_{t}\left(\operatorname{vec}[n]\left(a\left(t \boldsymbol{\Xi}_{n}^{(1)}\right)\right)_{k}\right)\right\} \leq \delta+D .
$$

Finally, set $\Delta=\delta+D$ and observe once more that since vec $[n]$ preserves the entries of matrices it must preserve the degrees of the entries. Therefore, for all $n \in \mathbb{Z}^{+}$,

$$
\max \left\{\operatorname{deg}_{t}\left(a_{k}\left(t \boldsymbol{\Xi}_{n}^{(1)}\right)_{i, j}\right): 1 \leq k \leq g, 1 \leq i, j \leq n\right\} \leq \Delta .
$$

Theorem 6.13. Suppose $a$ is hyporational. If $\operatorname{dom}_{n}(a)=M_{n}(\mathbb{C})^{g}$ for all $n$, then $a$ is a free polynomial. 
Proof. The hyporationality of $a$ implies $\left.a\right|_{M_{n}(\mathbb{C})^{g}}$ is a matrix of commutative rational

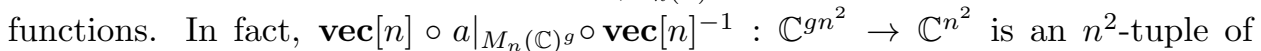
rational functions in $g n^{2}$ commuting indeterminates. Since $\operatorname{dom}_{n}(a)=M_{n}(\mathbb{C})^{g}$, each $\left(\left.\operatorname{vec}[n] \circ a\right|_{M_{n}(\mathbb{C})^{g}} \circ \mathbf{v e c}[n]^{-1}\right)_{k}$ is a rational function with domain of $\mathbb{C}^{g n^{2}}$, hence each is a polynomial. Thus, each $a\left(\boldsymbol{\Xi}_{n}^{(1)}\right)_{i, j}$ is a polynomial and in particular, $a\left(\boldsymbol{\Xi}_{n}^{(1)}\right)$ is a polynomial. However, Lemma 6.12 tells us that there is some $\Delta \in \mathbb{Z}^{+}$ such that for all $n \in \mathbb{Z}^{+}, \max _{i, j, k}\left\{\operatorname{deg}_{t}\left(a_{k}\left(t \boldsymbol{\Xi}_{n}^{(1)}\right)_{i, j}\right)\right\} \leq \Delta$. That is, $a$ is a free analytic function such that $a\left(\boldsymbol{\Xi}_{n}^{(1)}\right)$ is a polynomial for each $n$, and the degree of the polynomials is bounded. Therefore, Lemma 5.10 implies $a$ is a free polynomial.

Corollary 6.14. A free polynomial $p: M(\mathbb{C})^{g} \rightarrow M(\mathbb{C})^{g}$ is bijective if and only if $(\boldsymbol{h}, \boldsymbol{x}) \mapsto(D p(\boldsymbol{x})[\boldsymbol{h}], \boldsymbol{x})$ has a polynomial inverse.

Proof. Suppose $p: M(\mathbb{C})^{g} \rightarrow M(\mathbb{C})^{g}$ is a free polynomial and $F$ is its scion. Recall $F(\boldsymbol{x}, \boldsymbol{y})=(D p(\boldsymbol{y})[\boldsymbol{x}], \boldsymbol{y})$. If $F$ has a free polynomial inverse then $F$ is bijective. Hence Proposition 4.10 implies $p$ is bijective.

Suppose $p$ is bijective. An application of Proposition 4.10 shows $F$ is bijective. Let $G$ be the inverse of $F$. Theorem 3.17 implies $J_{F}$, the Jacobian matrix of $F$ is invertible as a polynomial matrix. Hence, $\mathbb{G}(\boldsymbol{x}, \boldsymbol{y})[\boldsymbol{z}, \boldsymbol{y}]=(\boldsymbol{x}, \boldsymbol{y}) J_{F}^{-1}(\boldsymbol{z}, \boldsymbol{y})$, the auxiliary inverse of $F$, must be a polynomial and by Lemma 4.11, $\mathbb{G}$ is a hyporealization. Thus, $G$ is the solution of the hyporealization $\mathbb{G}(\boldsymbol{x}, \boldsymbol{y})[\boldsymbol{z}, \boldsymbol{y}]$ and $G$ is hyporational.

Since $F$ is bijective and $G$ is its inverse, Lemma 3.15 says $\left.G\right|_{M_{n}(\mathbb{C})^{g}}$ agrees with a free polynomial, for each $n \in \mathbb{Z}^{+}$. In particular, $\operatorname{dom}_{n}(G)=M_{n}(\mathbb{C})^{g}$ for each $n \in \mathbb{Z}^{+}$. Thus, Theorem 6.13 implies $G$ is a free polynomial.

Theorem A - the Free Jacobian conjecture - tells us that a free polynomial $p$ is injective if and only if $D p(Y)[X]$ is nonsingular for all $X \in M(\mathbb{C})^{g}$. Corollary 6.14 strengthens this condition.

6.3. Bijectivity criteria. Proposition 4.10 tells us that a free polynomial is injective if and only if its scion is injective. Thus, when testing the bijectivity of a free polynomial, it suffices to only test for the bijectivity of its scion. The main result of this subsection, Theorem 6.18, combines Corollary 6.14 with Pascoe's Free Jacobian conjecture to get a more direct analog to the classical Jacobian conjecture.

Let $f \in(\mathbb{C}\langle y \cup \mathbb{x}\rangle)^{g}$. We say $f$ is $\boldsymbol{x}$-linear if $|w|_{x}=1$ for all monomials $w$ appearing in $f$. In other words, $f$ is a sum of monomials that contain exactly one $\boldsymbol{x}$-term.

Lemma 6.15. Suppose $f \in(\mathbb{C}\langle\mathbb{y} \cup \mathbb{x}\rangle)^{g}$. If $f$ is $\boldsymbol{x}$-linear, then there exists a matrix of bipartite polynomials, $\mathcal{J} \in M_{g}(\mathbb{C}\langle\mathbb{Z} \leftrightarrow \mathbb{y}\rangle)$, such that

$$
\operatorname{vec}[n](f(Y)[X])=\operatorname{vec}[n](X) \cdot \mathcal{J}\left(Y^{T} \otimes I_{n}, I_{n} \otimes Y\right),
$$

for all $X, Y \in M_{n}(\mathbb{C})^{g}$ and $n \in \mathbb{Z}^{+}$.

Proof. We omit the details of the construction of $\mathcal{J}$ since it is almost exactly the same as the construction of the hypomatrix representation found in Proposition 6.8. 
Definition 6.16. Suppose $p: M(\mathbb{C})^{g} \rightarrow M(\mathbb{C})^{g}$ is a free polynomial with derivative, $D p(\boldsymbol{y})[\boldsymbol{x}] \in(\mathbb{C}\langle\mathbf{y} \cup \boldsymbol{x}\rangle)^{g}$. Since $D p(\boldsymbol{y})[\boldsymbol{x}]$ is $\boldsymbol{x}$-linear, Lemma 6.15 implies there exists a matrix, $J_{p}^{\text {hyp }} \in M_{g}(\mathbb{C}\langle\mathbb{Z} \leftrightarrow \mathbb{y}\rangle)$, such that

$$
\operatorname{vec}[n](D p(Y)[X])=\operatorname{vec}[n](X) \cdot J_{p}^{\text {hyp }}\left(Y^{T} \otimes I_{n}, I_{n} \otimes Y\right),
$$

for all $X, Y \in M_{n}(\mathbb{C})^{g}$ and $n \in \mathbb{Z}^{+}$. We define $J_{p}^{\text {hyp }}$ to be the hypo-Jacobian matrix of $p$. The hypo-Jacobian matrix is unique.

Remark 6.17. Hypo-Jacobian matrices satisfy the chain rule. Namely,

$$
J_{\alpha \circ \beta}^{\text {hyp }}(\boldsymbol{z}, \boldsymbol{y})=J_{\beta}^{\text {hyp }}(\boldsymbol{z}, \boldsymbol{y}) J_{\alpha}^{\text {hyp }}\left(\beta^{T}(\boldsymbol{z}), \beta(\boldsymbol{y})\right),
$$

for all $\alpha, \beta \in\left(\mathbb{C}\langle\mathbb{x}\rangle_{+}\right)^{g}$.

Any endomorphism of the free associative algebra $\mathbb{C}\langle\mathbb{x}\rangle$ has a Jacobian matrix (see [DL82] and [Sch85]) that exactly corresponds with the hypo-Jacobian matrix found in this section. The Jacobian matrix of an endomorphism is a matrix over $\mathbb{C}\langle\mathbb{Z}\rangle^{o p p} \otimes \mathbb{C}\langle\mathbb{x}\rangle$, where $\mathbb{C}\langle\mathbb{Z}\rangle^{o p p}$ is the opposite ring of $\mathbb{C}\langle\mathbb{Z}\rangle$ (the order of multiplication is reversed). The construction of the hypo-Jacobian matrix sends terms of the form $U(\boldsymbol{y}) x_{i} V(\boldsymbol{y})$ to $U^{T}(\boldsymbol{z}) \otimes V(\boldsymbol{y})$. Since we can view the map $U \mapsto U^{T}$ as the canonical anti-isomorphism from $\mathbb{C}\langle\mathbb{Z}\rangle \rightarrow \mathbb{C}\langle\mathbb{Z}\rangle^{o p p}$, we see that the hypo-Jacobian matrix of a polynomial mapping and the Jacobian matrix of an endomorphism of $\mathbb{C}\langle\mathbb{x}\rangle$ are indeed the same.

Theorem 6.18. The free polynomial mapping $p$ is injective if and only if $J_{p}^{\text {hyp }}$ has a multiplicative inverse whose entries are bipartite polynomials.

Proof. Suppose $p$ is injective, $q$ is its inverse and $F=(D p(\boldsymbol{y})[\boldsymbol{x}], \boldsymbol{y})$ is the scion of $p$. Letting $G$ be the inverse of $F$, Lemma 4.11 shows $G(\boldsymbol{x}, \boldsymbol{y})=(D q(p(\boldsymbol{y}))[\boldsymbol{x}], \boldsymbol{y})$. Corollary 6.14 implies $G$ is a free polynomial, hence $D q(p(\boldsymbol{y}))[\boldsymbol{x}]$ is a free polynomial. Since $D q(p(\boldsymbol{y}))[\boldsymbol{x}]$ is $\boldsymbol{x}$-linear, Lemma 6.15 implies there exists a matrix $\mathcal{J} \in$ $M_{g}(\mathbb{C}\langle\mathbb{Z} \leftrightarrow \mathbb{y}\rangle)$, such that

$$
\operatorname{vec}[n](D q(p(Y))[X])=\operatorname{vec}[n](X) \cdot \mathcal{J}\left(Y^{T} \otimes I_{n}, I_{n} \otimes Y\right) .
$$

The chain rule tells us $D q \circ p(\boldsymbol{y})[\boldsymbol{x}]=D q(p(\boldsymbol{y}))[D p(\boldsymbol{y})[\boldsymbol{x}]]=\boldsymbol{x}$, thus

$$
\begin{aligned}
\operatorname{vec}[n](X) & =\operatorname{vec}[n](D p(Y)[X]) \cdot \mathcal{J}\left(Y^{T} \otimes I_{n}, I_{n} \otimes Y\right) \\
& =\operatorname{vec}[n](X) \cdot\left(J_{p}^{\text {hyp }} \cdot \mathcal{J}\right)\left(Y^{T} \otimes I_{n}, I_{n} \otimes Y\right) .
\end{aligned}
$$

Next, $D p \circ q(p(\boldsymbol{y}))[\boldsymbol{x}]=D p(\boldsymbol{y})[D q(p(\boldsymbol{y}))[\boldsymbol{x}]]=\boldsymbol{x}$, hence

$$
\begin{aligned}
\operatorname{vec}[n](X) & =\operatorname{vec}[n](D q(p(Y))[X]) \cdot J_{p}^{\mathrm{hyp}}\left(Y^{T} \otimes I_{n}, I_{n} \otimes Y\right) \\
& =\operatorname{vec}[n](X) \cdot\left(\mathcal{J} \cdot J_{p}^{\mathrm{hyp}}\right)\left(Y^{T} \otimes I_{n}, I_{n} \otimes Y\right) .
\end{aligned}
$$

Thus,

$$
\begin{aligned}
\operatorname{vec}[n](X) & =\operatorname{vec}[n](X) \cdot\left(J_{p}^{\text {hyp }} \cdot \mathcal{J}\right)\left(Y^{T} \otimes I_{n}, I_{n} \otimes Y\right) \\
& =\operatorname{vec}[n](X) \cdot\left(\mathcal{J} \cdot J_{p}^{\text {hyp }}\right)\left(Y^{T} \otimes I_{n}, I_{n} \otimes Y\right),
\end{aligned}
$$

for all $X, Y \in M_{n}(\mathbb{C})^{g}$, and $n \in \mathbb{Z}^{+}$. In other words, $\left(J_{p}^{\text {hyp }}\right)^{-1}=\mathcal{J} \in M_{g}(\mathbb{C}\langle\mathbb{Z} \leftrightarrow \mathbb{y}\rangle)$.

Conversely suppose $\left(J_{p}^{\text {hyp }}\right)^{-1} \in M_{g}(\mathbb{C}\langle\mathbb{Z} \leftrightarrow \mathbb{y}\rangle)$. Let $\hat{G}$ be the free polynomial defined by

$$
\hat{G}(Y)[X]=\operatorname{vec}[n]^{-1}\left(\operatorname{vec}[n](X) \cdot\left(J_{p}^{\mathrm{hyp}}\right)^{-1}\left(Y^{T} \otimes I_{n}, I_{n} \otimes Y\right)\right),
$$


for all $X, Y \in M_{n}(\mathbb{C})^{g}$ and $n \in \mathbb{Z}^{+}$. Observe

$$
\begin{aligned}
\operatorname{vec}[n](X) & =\operatorname{vec}[n](X) \cdot\left(J_{p}^{\text {hyp }} \cdot\left(J_{p}^{\text {hyp }}\right)^{-1}\right)\left(Y^{T} \otimes I_{n}, I_{n} \otimes Y\right) \\
& =\operatorname{vec}[n](D p(Y)[X]) \cdot\left(J_{p}^{\text {hyp }}\right)^{-1}\left(Y^{T} \otimes I_{n}, I_{n} \otimes Y\right) \\
& =\operatorname{vec}[n](\hat{G}(Y)[D p(Y)[X]]) .
\end{aligned}
$$

Hence, $X=\hat{G}(Y)[D p(Y)[X]]$ and $\boldsymbol{x}=\hat{G}(\boldsymbol{y})[D p(\boldsymbol{y})[\boldsymbol{x}]]$. On the other hand,

$$
\begin{aligned}
\operatorname{vec}[n](X) & =\operatorname{vec}[n](X) \cdot\left(\left(J_{p}^{\mathrm{hyp}}\right)^{-1} \cdot J_{p}^{\mathrm{hyp}}\right)\left(Y^{T} \otimes I_{n}, I_{n} \otimes Y\right) \\
& =\operatorname{vec}[n](\hat{G}(Y)[X]) \cdot J_{p}^{\mathrm{hyp}}\left(Y^{T} \otimes I_{n}, I_{n} \otimes Y\right) \\
& =\operatorname{vec}[n](D p(Y)[\hat{G}(Y)[X]]) .
\end{aligned}
$$

Hence, $X=D p(Y)[\hat{G}(Y)[X]]$ and consequently, $\boldsymbol{x}=D p(\boldsymbol{y})[\hat{G}(\boldsymbol{y})[\boldsymbol{x}]]$. By setting $G(\boldsymbol{x}, \boldsymbol{y})=(\hat{G}(\boldsymbol{y})[\boldsymbol{x}], \boldsymbol{y})$, we get

$$
G(F(\boldsymbol{x}, \boldsymbol{y}))=G(D p(\boldsymbol{y})[\boldsymbol{x}], \boldsymbol{y})=(\hat{G}(\boldsymbol{y})[D p(\boldsymbol{y})[\boldsymbol{x}]], \boldsymbol{y})=(\boldsymbol{x}, \boldsymbol{y}),
$$

and

$$
F(G(\boldsymbol{x}, \boldsymbol{y}))=F(\hat{G}(\boldsymbol{y})[\boldsymbol{x}], \boldsymbol{y})=(D p(\boldsymbol{y})[\hat{G}(\boldsymbol{y})[\boldsymbol{x}]], \boldsymbol{y})=(\boldsymbol{x}, \boldsymbol{y}) .
$$

Thus, $G$ is the inverse of $F$. Therefore, by Corollary $6.14, p$ is an injective free polynomial.

Before we finally move on to the proof of Theorem 6.22 we connect the composition of polynomial mappings to the composition of endomorphisms of $\mathbb{C}\langle\mathbb{x}\rangle$.

Definition 6.19. Suppose $p \in(\mathbb{C}\langle\mathbb{x}\rangle)^{g}$ is a free polynomial mapping and let $\phi: \mathbb{C}\langle\mathbb{x}\rangle \rightarrow \mathbb{C}\langle\mathbb{x}\rangle$ be an algebra homomorphism. We say $\phi$ is induced by $p$ if $\phi\left(x_{i}\right)=p_{i}(\boldsymbol{x})$, for $1 \leq i \leq g$. Similarly, we say $p$ is induced by $\phi$ if $p(\boldsymbol{x})=$ $\left(\phi\left(x_{1}\right), \ldots, \phi\left(x_{g}\right)\right)$.

Lemma 6.20. Suppose $\phi, \psi: \mathbb{C}\langle\mathbb{x}\rangle \rightarrow \mathbb{C}\langle\mathbb{x}\rangle$ are algebra homomorphisms. If $p, q$ are the induced polynomial mappings of $\phi$ and $\psi$, respectively, then

$$
(q \circ p)(\boldsymbol{x})=\left((\phi \circ \psi)\left(x_{1}\right), \ldots,(\phi \circ \psi)\left(x_{g}\right)\right) .
$$

Proof. This is verified rather easily from definitions. The details are left to the reader.

If $\phi$ is an endomorphism of $\mathbb{C}\langle\mathbb{x}\rangle$ then the Jacobian matrix of $\phi$ is a $g \times g$ matrix over $\mathbb{C}\langle\mathbb{Z}\rangle^{o p p} \otimes \mathbb{C}\langle\mathbb{x}\rangle$. More specifically, if $p$ is the polynomial mapping induced by $\phi$, then the Jacobian matrix of $\phi$ is found by applying the natural anti-isomorphism $M_{g}(\mathbb{C}\langle\mathbb{Z}\rangle \otimes \mathbb{C}\langle\mathbb{x}\rangle) \rightarrow M_{g}\left(\mathbb{C}\langle\mathbb{Z}\rangle^{\text {opp }} \otimes \mathbb{C}\langle\mathbb{x}\rangle\right)$ to $J_{p}^{\text {hyp }}$.

Theorem 6.21. Suppose $p: M(\mathbb{C})^{g} \rightarrow M(\mathbb{C})^{g}$ is a free polynomial mapping. The following are equivalent;

(i) $p$ is injective;

(ii) $p$ is bijective;

(iii) $D p(Y)$ is a nonsingular map for all $Y \in M_{n}(\mathbb{C})^{g}$ and all $n \in \mathbb{Z}^{+}$;

(iv) $J_{p}^{\text {hyp }}$ is invertible;

(v) $p^{-1}$ exists and is a free polynomial. 
Proof. (i) $\Leftrightarrow($ ii $) \Leftrightarrow($ iii) is Theorem A. (i) $\Leftrightarrow$ (iv) is Theorem 6.18. (v) $\Rightarrow$ (i) is clear.

To show (iv) $\Rightarrow(\mathrm{v})$, we assume $J_{p}^{\text {hyp }}$ is invertible. Let $\phi$ be the endomorphism of $\mathbb{C}\langle\mathbb{x}\rangle$ induced by $p$ (so $\phi\left(x_{i}\right)=p_{i}$ for $1 \leq i \leq g$ ). Since $J_{p}^{\text {hyp }}$ is invertible, it follows that that Jacobian matrix of $\phi$ is invertible. Thus, Proposition 3.1 in [DL82] implies $\phi$ is an epic endomorphism and Theorem 12.7 in [Sch85] implies $\phi$ is an automorphism of $\mathbb{C}\langle\mathbb{x}\rangle$. So $\phi^{-1}$ exists and is an automorphism itself.

Let $q=\left(\phi^{-1}\left(x_{1}\right), \ldots, \phi^{-1}\left(x_{g}\right)\right)$ be polynomial mapping induced by $\phi^{-1}$. By Lemma 6.20,

$$
\left(x_{1}, \ldots, x_{g}\right)=\left(\left(\phi \circ \phi^{-1}\right)\left(x_{1}\right), \ldots,\left(\phi \circ \phi^{-1}\right)\left(x_{g}\right)\right)=(q \circ p)(\boldsymbol{x})
$$

and

$$
\left(x_{1}, \ldots, x_{g}\right)=\left(\left(\phi^{-1} \circ \phi\right)\left(x_{1}\right), \ldots,\left(\phi^{-1} \circ \phi\right)\left(x_{g}\right)\right)=(p \circ q)(\boldsymbol{x}) .
$$

Thus, $p$ and $q$ are inverse mappings. Therefore, $p$ is injective.

Theorem 6.22 ((Free Grothendieck Theorem)). If $p: M(\mathbb{C})^{g} \rightarrow M(\mathbb{C})^{g}$ is an injective free polynomial mapping, then there is a free polynomial mapping $q$ such that $p \circ q(x)=x=q \circ p(x)$; that is, $p$ has a free polynomial inverse.

Proof. This is exactly (i) $\Rightarrow(v)$ in Theorem 6.21.

\section{Computing inverses}

Suppose $p: M(\mathbb{C})^{g} \rightarrow M(\mathbb{C})^{g}$ is a free polynomial, $F$ is its scion and $q$ and $G$ are the inverses (when they exist) of $p$ and $F$, respectively. By either Corollary 6.14 or Theorem 6.18 we know that $p$ is injective if and only if $G$ is free polynomial. Recall that if $p$ has a free polynomial inverse $q$, then Lemma 4.11 tells us that $\operatorname{deg}(q) \leq \operatorname{deg}(G) \leq \operatorname{deg}(p) \operatorname{deg}(q)$. Thus, an upper bound on $\operatorname{deg}(G)$ gives us an upper bound on the possible degree of $q$.

Definition 7.1. Let $V_{t}(0)=0$ and for any nonzero rational function $r \in \mathbb{C} \nless \mathbb{x} \ngtr$ define

$$
V_{t}(r(t \boldsymbol{x}))=\min \left\{\max \left\{\operatorname{deg}_{t}(\alpha(\boldsymbol{x})[t]), \operatorname{deg}_{t}(\beta(\boldsymbol{x})[t])\right\}: r(t \boldsymbol{x})=\alpha(\boldsymbol{x})[t] \beta(\boldsymbol{x})[t]^{-1}\right\} .
$$

If $M \in M_{m \times n}(\mathbb{C} \nless \mathbb{x} \ngtr)$ then define

$$
V_{t}(M(t \boldsymbol{x}))=\max \left\{V_{t}\left(M(t \boldsymbol{x})_{i, j}\right): 1 \leq i \leq m, 1 \leq j \leq n\right\} .
$$

Note that if $r \in \mathbb{C}\langle\mathbb{x}\rangle$ then $V_{t}(r(t \boldsymbol{x}))=\operatorname{deg}(r(\boldsymbol{x}))$.

Remark 7.2. It is straightforward to see that $\left|\operatorname{deg}_{t}(r(t \boldsymbol{x}))\right| \leq V_{t}(r(t \boldsymbol{x}))$, for any nonzero rational function. By appealing to evaluations on generic matrices we get that

and

$$
V_{t}(r(t \boldsymbol{x}) s(t \boldsymbol{x})) \leq V_{t}(r(t \boldsymbol{x}))+V_{t}(s(t \boldsymbol{x}))
$$

$$
V_{t}(r(t \boldsymbol{x})+s(t \boldsymbol{x})) \leq V_{t}(r(t \boldsymbol{x}))+V_{t}(s(t \boldsymbol{x})) .
$$

Hence, if $M \in M_{\ell \times m}(\mathbb{C} \nless \mathbb{x} \ngtr)$ and $N \in M_{m \times n}(\mathbb{C} \nless \mathbb{x} \ngtr)$ then

$$
\begin{aligned}
V_{t}(M(t \boldsymbol{x}) N(t \boldsymbol{x})) & \leq \max _{i, k}\left\{\sum_{j=1}^{m} V_{t}\left(M(t \boldsymbol{x})_{i, j} N(t \boldsymbol{x})_{j, k}\right)\right\} \\
& \leq \max _{i, k}\left\{\sum_{j=1}^{m} V_{t}\left(M(t \boldsymbol{x})_{i, j}\right)+V_{t}\left(N(t \boldsymbol{x})_{j, k}\right)\right\}
\end{aligned}
$$




$$
\leq m \cdot V_{t}\left(M(t \boldsymbol{x})+m \cdot V_{t}(N(t \boldsymbol{x}))\right) .
$$

Lemma 7.3. Let $\mathfrak{f}(1)=1$ and for any integer $n \geq 1$, define $\mathfrak{f}(n+1)=(n+1)^{3} \mathfrak{f}(n)$. If $M, M^{-1} \in M_{n}(\mathbb{C} \nless \mathbb{x} \ngtr)$ then $\left|\operatorname{deg}_{t}\left(M^{-1}(t \boldsymbol{x})\right)\right| \leq 3^{n} \mathfrak{f}(n) V_{t}(M(t \boldsymbol{x}))$.

Proof. We claim $V_{t}\left(M^{-1}(t \boldsymbol{x})\right) \leq 3^{n} \mathfrak{f}(n) V_{t}(M(t \boldsymbol{x}))$ and use induction to prove the claim.

If $n=1$ then $M, M^{-1} \in \mathbb{C} \nless \mathbb{x} \ngtr$ and $\operatorname{deg}_{t}\left(M(t \boldsymbol{x})^{-1}\right)=-\operatorname{deg}_{t}(M(t \boldsymbol{x}))$, hence $V_{t}\left(M(t \boldsymbol{x})^{-1}\right)=V_{t}(M(t \boldsymbol{x})) \leq 3 V_{t}(M(t \boldsymbol{x}))$. Write

Now suppose the statement holds for $n$ and consider $M, M^{-1} \in M_{n+1}(\mathbb{C} \nless \mathbb{x} \ngtr)$.

$$
M=\left(\begin{array}{ll}
A & b \\
c & d
\end{array}\right)
$$

where $A \in M_{n}(\mathbb{C} \nless \mathbb{x} \ngtr), b \in M_{n \times 1}(\mathbb{C} \nless \mathbb{x} \ngtr), c \in M_{1 \times n}(\mathbb{C} \nless \mathbb{x} \ngtr)$, and $d \in \mathbb{C} \nless \mathbb{x} \ngtr$.

If $S \in \mathrm{GL}_{n+1}(\mathbb{C})$ then $M S$ is invertible. Hence, we may assume $d$ is nonzero and thus, $d^{-1}$ exists. Observe

$$
V_{t}\left(A(t \boldsymbol{x})-b(t \boldsymbol{x}) d(t \boldsymbol{x})^{-1} c(t \boldsymbol{x})\right) \leq V_{t}(A)+V_{t}\left(b d^{-1} c\right) \leq(3 n+1) V_{t}(M(t \boldsymbol{x})) .
$$

Hence

$$
V_{t}\left(\left(A-b d^{-1} c\right)^{-1}\right) \leq 3^{n}(3 n+1) \mathfrak{f}(n) V_{t}(M) .
$$

Set $N(t \boldsymbol{x})=\left(A(t \boldsymbol{x})-b(t \boldsymbol{x}) d(t \boldsymbol{x})^{-1} c(t \boldsymbol{x})\right)^{-1}$ and observe

$$
\begin{aligned}
V_{t}\left(d^{-1}\left(1+c N b d^{-1}\right)\right) & \leq V_{t}(M)+V_{t}\left(1+c N b d^{-1}\right)=2 V_{t}(M)+V_{t}(c N b) \\
& \leq 2 V_{t}(M)+\sum_{i=1}^{n}\left(V_{t}\left(c_{i}\right)\right)+\sum_{j, k=1}^{n}\left(V_{t}\left(N_{j, k}\right)+V_{t}\left(b_{k}\right)\right) \\
& \leq 2(n+1) V_{t}(M)+n^{2} V_{t}(N)
\end{aligned}
$$

Applying the induction hypothesis,

$$
\begin{aligned}
2(n+1) V_{t}(M)+n^{2} V_{t}(N) & \leq(2 n+1) V_{t}(M)+n^{2}\left(3^{n}(3 n+1) \mathfrak{f}(n) V_{t}(M)\right) \\
& =\left(\left(3^{n+1} n^{3}+3^{n} n^{2}\right) \mathfrak{f}(n)+2 n+2\right) V_{t}(M) \\
& \leq 3^{n+1}\left(n^{3}+n^{2}+2 n+2\right) \mathfrak{f}(n) V_{t}(M) \\
& \leq 3^{n+1}(n+1)^{3} \mathfrak{f}(n) V_{t}(M)=3^{n+1} \mathfrak{f}(n+1) V_{t}(M) .
\end{aligned}
$$

Since the inverse of $M$ is determined from the Schur complement, we have proven that $V_{t}\left(M^{-1}(t \boldsymbol{x})\right) \leq 3^{n} \mathfrak{f}(n) V_{t}(M(t \boldsymbol{x}))$.

Finally, since $\left|\operatorname{deg}_{t}(r(t \boldsymbol{x}))\right| \leq V_{t}(r(t \boldsymbol{x}))$ for any nonzero rational $r$, we have

$$
\left|\operatorname{deg}_{t}\left(M^{-1}(t \boldsymbol{x})\right)\right| \leq 3^{n} \mathfrak{f}(n) V_{t}(M(t \boldsymbol{x})),
$$

as desired.

Lemma 7.4. If $M, M^{-1} \in M_{n}(\mathbb{C}\langle\mathbb{x}\rangle)$ then $\operatorname{deg}\left(M^{-1}\right) \leq 3^{n} \mathfrak{f}(n) \operatorname{deg}(M)$.

Proof. Since $M$ and $M^{-1}$ are matrices of polynomials, $V_{t}(M(t \boldsymbol{x}))=\operatorname{deg}(M(t \boldsymbol{x}))$ and $V_{t}\left(M(t \boldsymbol{x})^{-1}\right)=\operatorname{deg}\left(M(t \boldsymbol{x})^{-1}\right)$. Thus, by Lemma 7.3,

$$
\operatorname{deg}\left(M(t \boldsymbol{x})^{-1}\right)=\left|\operatorname{deg}_{t}\left(M(t \boldsymbol{x})^{-1}\right)\right| \leq 3^{n} \mathfrak{f}(n) V_{t}(M)=3^{n} \mathfrak{f}(n) \operatorname{deg}(M) .
$$


The degree bound in Lemma 7.4 is far from optimal. However, to improve the degree bound in a significant manner would require an altogether different proof; the induction hypothesis cannot be applied to $\left(A-b d^{-1} c\right)^{-1}$ since it is not necessarily the inverse of a polynomial matrix.

Suppose $\mathcal{B}: \mathbb{N} \times \mathbb{N} \rightarrow \mathbb{N}$ is a function such that whenever $M \in M_{n}(\mathbb{C}\langle\mathbb{x}\rangle)$ and $M^{-1} \in M_{n}(\mathbb{C}\langle\mathbf{x}\rangle)$, we have $\operatorname{deg}\left(M^{-1}\right) \leq \mathcal{B}(n, \operatorname{deg}(M))$. We call such a function a PMID bound (for Polynomial Matrix Inverse Degree).

Theorem 7.5. Suppose $\mathcal{B}$ is a PMID bound. Let $p$ be a bijective free polynomial and let $q$ be its inverse. If $q$ is a free polynomial then $\operatorname{deg}(q) \leq \mathcal{B}(g, \operatorname{deg}(p)-1)+1$.

Proof. We begin by noting that $\operatorname{deg}(D p(\boldsymbol{y})[\boldsymbol{x}])=\operatorname{deg}(p)$. Since $J_{p}^{\text {hyp }}$, the hypoJacobian of $p$, is constructed from $D p(\boldsymbol{y})[\boldsymbol{x}]$, we get that $\operatorname{deg}\left(J_{p}^{\text {hyp }}\right)=\operatorname{deg}(p)-1$. By Theorem 6.21 we know $\left(J_{p}^{\text {hyp }}\right)^{-1}$ is a polynomial matrix since $p$ is injective. Hence, $\operatorname{deg}\left(\left(J_{p}^{\text {hyp }}\right)^{-1}\right) \leq \mathcal{B}(g, \operatorname{deg}(p)-1)$. In fact, for all $n \in \mathbb{Z}^{+}$and $X, Y \in M_{n}(\mathbb{C})^{g}$,

$$
\operatorname{vec}[n](D q(p(Y))[X])=\operatorname{vec}[n](X) \cdot\left(J_{p}^{\text {hyp }}\right)^{-1}\left(Y^{T} \otimes I_{n}, I_{n} \otimes Y\right) .
$$

Thus, $\operatorname{deg}(D q(p(\boldsymbol{y}))[\boldsymbol{x}])=\operatorname{deg}\left(\left(J_{p}^{\mathrm{hyp}}\right)^{-1}\right)+1 \leq \mathcal{B}(g, \operatorname{deg}(p)-1)+1$. Lemma 4.11 says $G=(D q(p(\boldsymbol{y}))[\boldsymbol{x}], \boldsymbol{y})$, where $G$ is the inverse of $F$, the scion of $p$. In fact, $\operatorname{deg}(q) \leq \operatorname{deg}(G)$. Therefore

$$
\operatorname{deg}(q) \leq \operatorname{deg}(G)=\operatorname{deg}(D q(p(\boldsymbol{y}))[\boldsymbol{x}]) \leq \mathcal{B}(g, \operatorname{deg}(p)-1)+1,
$$

as desired.

Recall from Definition 3.10, $\mathbb{q}(\boldsymbol{x})[\boldsymbol{z}]=\boldsymbol{x} J_{p}(\boldsymbol{z})$ and $\mathbb{q}^{\circ+1}(\boldsymbol{x})[\boldsymbol{z}]=\mathrm{q}^{\circ k}(\boldsymbol{x})[\mathrm{q}(\boldsymbol{x})[\boldsymbol{z}]]$. For each $k$, we write

$$
\mathrm{qI}^{\circ k}(\boldsymbol{x})[\boldsymbol{z}]=\sum_{w \in\langle\mathbb{x} \cup \mathbb{Z}\rangle} \rho_{w}^{k} w,
$$

set $\mathbb{d}_{q}^{k}=\mathbb{d}_{z}\left(\mathfrak{q}^{\circ k}\right)=\inf \left\{|w|:|w|_{z}>0\right.$ and $\left.\rho_{w}^{k} \neq 0\right\}$ and write $\mathfrak{q}^{\circ k}(\boldsymbol{x})[\boldsymbol{z}]=\mathfrak{q}^{k}(\boldsymbol{x})+$ $r^{k}(\boldsymbol{x})[\boldsymbol{z}]$, where

$$
\mathfrak{q}^{k}(\boldsymbol{x})=\sum_{\substack{w \in\langle\mathbb{x}\rangle \\|w|<\mathbb{d}_{q}^{k}}} \rho_{w}^{k} w \quad \text { and } \quad r^{k}(\boldsymbol{x})[\boldsymbol{z}]=\sum_{\substack{w \in\langle\mathbb{x} \cup \mathbb{Z}\rangle \\|w| \geq \mathbb{d}_{q}^{k}}} \rho_{w}^{k} w .
$$

From Lemma 3.9 we know $\mathbb{d}_{z}^{k}>k$ and $\left(\mathfrak{q}^{k}\right)_{k=1}^{\infty}$ is a sequence of polynomials converging to $q$ such that $\operatorname{deg}\left(\mathfrak{q}^{k}\right) \nearrow \operatorname{deg}(q)$. Moreover, Lemma 4.1 shows that $q$ is a polynomial if and only if there exists an $N$ such that $\operatorname{deg}(p) \operatorname{deg}\left(\mathfrak{q}^{N}\right)<\mathbb{d}_{z}^{N}$.

Lemma 7.6. Suppose $\mathcal{B}$ is a PMID bound. Let $p \in\left(\mathbb{C}\langle\mathbb{x}\rangle_{+}\right)^{g}$ and let $q$ be the compositional inverse of $p$. Set $B=\mathcal{B}(g, \operatorname{deg}(p)-1)+1$. Then $q$ is a free polynomial if and only if $J_{p}^{-1} \in M_{g}(\mathbb{C}\langle\mathbb{x}\rangle)$ and $\operatorname{deg}\left(\mathfrak{q}^{k}\right) \leq B$ for all $k$.

Proof. Suppose $J_{p}^{-1} \in M_{g}(\mathbb{C}\langle\mathbb{x}\rangle)$ and $\operatorname{deg}\left(\mathfrak{q}^{k}\right) \leq B$ for all $k$. In particular, $\operatorname{deg}\left(\mathfrak{q}^{B \operatorname{deg}(p)}\right) \leq B$, hence $\operatorname{deg}\left(\mathfrak{q}^{B \operatorname{deg}(p)}\right) \operatorname{deg}(p) \leq B \operatorname{deg}(p)<\mathbb{d}_{q}^{B \operatorname{deg}(p)}$ and $q$ is a polynomial by Lemma 4.1 .

Conversely suppose $q$ is a polynomial. It follows that $q(X)$ exists for all $X \in$ $M(\mathbb{C})^{g}$, and consequently $p(q(X))=X=q(p(X))$ for all $X \in M(\mathbb{C})^{g}$. Thus, $p$ is bijective and Theorem 3.17 implies $J_{p}^{-1} \in M_{g}(\mathbb{C}\langle\mathbb{x}\rangle)$. Next, Theorem 7.5 implies $\operatorname{deg}(q) \leq \mathcal{B}(g, \operatorname{deg}(p)-1)+1=B$ and since $\operatorname{deg}\left(\mathfrak{q}^{k}\right) \leq \operatorname{deg}(q)$, we have exactly $\operatorname{deg}\left(\mathfrak{q}^{k}\right) \leq B$ for all $k$. 
Lemma 7.6 hints at a simple algorithm for determining whether $q$, the inverse of a given polynomial $p \in\left(\mathbb{C}\langle\mathbb{x}\rangle_{+}\right)^{g}$, is a polynomial. We still set $B=\mathcal{B}(g, \operatorname{deg}(p)-1)+1$. Corollary 7.7. Either $\mathfrak{q}^{B}=q$ or $q$ is not a polynomial and $p$ is not injective.

Proof. Let $q=\sum_{w} \rho_{w} w$. Recall $\operatorname{deg}\left(\mathfrak{q}^{k}\right)$ is an increasing sequence and $q$ and $\mathfrak{q}^{k}$ agree on monomials of length less than $\mathbb{d}_{q}^{k}$. If $\mathfrak{q}^{B} \neq q$ then there exists a $k>B$ and $w \in\langle\mathbb{x}\rangle$ with $|w|>\mathbb{d}_{q}^{B}>B$ such that $\rho_{w}^{k} \neq 0$. In particular $\operatorname{deg}\left(\mathfrak{q}^{k}\right)>B$, thus by Lemma 7.6, $q$ is not a polynomial and $p$ is not injective.

For any $p$, the algorithmic approach to computing $q$ is as follows.

- If: $J_{p} \notin M_{g}(\mathbb{C}\langle\mathbb{x}\rangle)$ then $q$ is not a polynomial;

- Else: compute $B=\mathcal{B}(g, \operatorname{deg}(p)-1)+1$;

- Set $k=1$ and $\mathbb{q}^{\circ 0}(\boldsymbol{x})[\boldsymbol{z}]=\boldsymbol{z}$;

- Loop:

$\sim$ Compute $\mathrm{q}^{\circ k}(\boldsymbol{x})[\boldsymbol{z}]=\mathrm{q}(\boldsymbol{x})\left[\mathrm{q}^{\circ k-1}(\boldsymbol{x})[\boldsymbol{z}]\right] ;$

$\sim$ If: $\operatorname{deg}\left(\mathfrak{q}^{k}\right)>B$ or $k>B$ then $q$ is not a polynomial;

$\sim$ ElseIf: $\operatorname{deg}\left(\mathfrak{q}^{k}\right) \operatorname{deg}(p)<\mathbb{d}_{q}^{k}$ then $\mathfrak{q}=q$;

$\sim$ Else: Increase $k$ by one.

In at most $B$ loops, the above algorithm would either tell us that $q$ is not a polynomial or would return a polynomial inverse $q$.

\section{REFERENCES}

[AM16] Jim Agler and John E. McCarthy. The implicit function theorem and free algebraic sets. Trans. Amer. Math. Soc., 368(5):3157-3175, 2016.

[BCW82] Hyman Bass, Edwin H. Connell, and David Wright. The Jacobian conjecture: Reduction of degree and formal expansion of the inverse. Bull. Amer. Math. Soc. (N.S.), 7(2):287330, 091982.

[BR11] J. Berstel and C. Reutenauer. Noncommutative Rational Series with Applications. Encyclopedia of Mathematics an. Cambridge University Press, 2011.

[Coh95] P.M. Cohn. Skew Fields: Theory of General Division Rings. Encyclopedia of Mathematics an. Cambridge University Press, 1995.

[Dic82] Warren Dicks. A commutator test for two elements to generate the free algebra of rank two. Bull. London Math. Soc., 14(1):48-51, 1982.

[DL82] Warren Dicks and Jacques Lewin. A jacobian conjecture for free associative algebras. Communications in Algebra, 10(12):1285-1306, 1982.

[DY06] Vesselin Drensky and Jie-Tai Yu. The strong Anick conjecture. Proc. Natl. Acad. Sci. USA, 103(13):4836-4840, 2006.

[DY07] Vesselin Drensky and Jie-Tai Yu. Tame automorphisms fixing a variable of free associative algebras of rank three. Internat. J. Algebra Comput., 17(5-6):999-1011, 2007.

[Gro66] A. Grothendieck. Éléments de géométrie algébrique (rédigé avec la collaboration de Jean Dieudonné): IV. Étude locale des schémas et des morphismes de schémas. III. Inst. Hautes Études Sci. Publ. Math., page 255, 1966.

[Gro67] A. Grothendieck. Éléments de géométrie algébrique (rédigé avec la collaboration de Jean Dieudonné): IV. Étude locale des schémas et des morphismes de schémas. IV. Inst. Hautes Études Sci. Publ. Math., 32:361, 1967.

[HJ94] R.A. Horn and C.R. Johnson. Topics in Matrix Analysis. Topics in Matrix Analysis. Cambridge University Press, 1994.

[HKM11] J. William Helton, Igor Klep, and Scott McCullough. Proper analytic free maps. Journal of Functional Analysis, 260(5):1476 - 1490, 2011.

[HKM12] J. William Helton, Igor Klep, and Scott McCullough. Free Analysis, Convexity and LMI Domains, pages 195-219. Springer Basel, Basel, 2012.

[HM04] J. William Helton and Scott McCullough. A positivstellensatz for non-commutative polynomials. Transactions of the American Mathematical Society, 356(9):3721-3737, 2004. 
[KPV17] Igor Klep, James Eldred Pascoe, and Jurij Volcic. Regular and positive noncommutative rational functions. J. Lond. Math. Soc. (2), 95(2):613-632, 2017.

[KŠ17] I. Klep and Š. Špenko. Free function theory through matrix invariants. Canad. J. Math., 69:408-433, 2017.

[KV17] I. Klep and J. Volčič. Free loci of matrix pencils and domains of noncommutative rational functions. Commentarii Mathematici Helvetici, 92(1):105-130, 2017.

[KVV14] D.S. Kaliuzhnyi-Verbovetskyi and V. Vinnikov. Foundations of Free Noncommutative Function Theory: Mathematical Surveys and Monographs. American Mathematical Society, 2014.

[KVV16] Igor Klep, Victor Vinnikov, and Jurij Volčič. Multipartite rational functions, 2016.

[ML70] L. G. Makar-Limanov. The automorphisms of the free algebra with two generators. Funkcional. Anal. i Priložen., 4(3):107-108, 1970.

[MR01] J.C. McConnel and J.C. Robson. Noncommutative Noetherian Rings. Wiley series in pure and applied mathematics. Wiley, 2001.

[Pas14] J. E. Pascoe. The inverse function theorem and the jacobian conjecture for free analysis. Mathematische Zeitschrift, 278(3):987-994, 2014.

[PS09] Ion Petre and Arto Salomaa. Algebraic Systems and Pushdown Automata, pages 257-289. Springer Berlin Heidelberg, Berlin, Heidelberg, 2009.

[Reu92] Christophe Reutenauer. Applications of a noncommutative jacobian matrix. Journal of Pure and Applied Algebra, 77(2):169 - 181, 1992.

[Sch85] A. H. Schofield. Representations of Rings over Skew Fields. London Mathematical Society Lecture Note Series. Cambridge University Press, 1985.

[SS78] Arto Salomaa and M. Soittola. Automata: Theoretic Aspects of Formal Power Series. Springer-Verlag New York, Inc., Secaucus, NJ, USA, 1978.

[Sta11] Richard P. Stanley. Enumerative Combinatorics: Volume 1. Cambridge University Press, New York, NY, USA, 2nd edition, 2011.

[SU03] Ivan P. Shestakov and Ualbai U. Umirbaev. The Nagata automorphism is wild. Proc. Natl. Acad. Sci. USA, 100(22):12561-12563, 2003.

[SU04] Ivan P. Shestakov and Ualbai U. Umirbaev. Poisson brackets and two-generated subalgebras of rings of polynomials. J. Amer. Math. Soc., 17(1):181-196, 2004.

[Umi06] U. U. Umirbaev. Defining relations of the tame automorphism group of polynomial algebras in three variables. J. Reine Angew. Math., 600:203-235, 2006.

[Umi07] U. U. Umirbaev. The Anick automorphism of free associative algebras. J. Reine Angew. Math., 605:165-178, 2007.

[UY04] Ualbai U. Umirbaev and Jie-Tai Yu. The strong Nagata conjecture. Proc. Natl. Acad. Sci. USA, 101(13):4352-4355, 2004.

[vdE00] Arno van den Essen. Polynomial automorphisms and the Jacobian conjecture, volume 190 of Progress in Mathematics. Birkhäuser Verlag, Basel, 2000.

[Vol15] Jurij Volčič. Matrix coefficient realization theory of noncommutative rational functions, 2015. 